\title{
Aspects of physiotherapy in the peri-operative management of total knee arthroplasty patients
}

Citation for published version (APA):

Lenssen, A. F. (2007). Aspects of physiotherapy in the peri-operative management of total knee arthroplasty patients. [Doctoral Thesis, Maastricht University]. Universiteit Maastricht. https://doi.org/10.26481/dis.20070907al

Document status and date:

Published: 01/01/2007

DOI:

10.26481/dis.20070907al

Document Version:

Publisher's PDF, also known as Version of record

\section{Please check the document version of this publication:}

- A submitted manuscript is the version of the article upon submission and before peer-review. There can be important differences between the submitted version and the official published version of record.

People interested in the research are advised to contact the author for the final version of the publication, or visit the DOI to the publisher's website.

- The final author version and the galley proof are versions of the publication after peer review.

- The final published version features the final layout of the paper including the volume, issue and page numbers.

Link to publication

\footnotetext{
General rights rights.

- You may freely distribute the URL identifying the publication in the public portal. please follow below link for the End User Agreement:

www.umlib.nl/taverne-license

Take down policy

If you believe that this document breaches copyright please contact us at:

repository@maastrichtuniversity.nl

providing details and we will investigate your claim.
}

Copyright and moral rights for the publications made accessible in the public portal are retained by the authors and/or other copyright owners and it is a condition of accessing publications that users recognise and abide by the legal requirements associated with these

- Users may download and print one copy of any publication from the public portal for the purpose of private study or research.

- You may not further distribute the material or use it for any profit-making activity or commercial gain

If the publication is distributed under the terms of Article $25 \mathrm{fa}$ of the Dutch Copyright Act, indicated by the "Taverne" license above, 


\begin{abstract}
Aspects of physiotherapy
in the peri-operative management of total knee arthroplasty patients
\end{abstract}

Ton Lenssen 



\section{Aspects of physiotherapy in the peri-operative management of total knee arthroplasty patients}

Proefschrift

ter verkrijging van de graad van doctor aan de Universiteit Maastricht, op gezag van de Rector Magnificus, prof. mr. G.P.M.F. Mols, volgens het besluit van het college van Decanen, in het openbaar te verdedigen op vrijdag 7 september 2007 om 14.00 uur

door

Antoine François Lenssen 


\section{Promotores:}

prof. dr. R.A. de Bie prof. dr. P.A. van den Brandt prof. dr. R.G.T. Geesink

\section{Beoordelingscommissie:}

prof. dr. J.M.J.M. van der Linden (voorzitter) prof. dr. G.J. Dinant prof. dr. H. Kuipers prof. dr. R.A.B. Oostendorp (UMC Nijmegen) prof. dr. J.A.N. Verhaar (Erasmus MC Rotterdam) 


\section{Contents}

$\begin{array}{lll}\text { Chapter 1. } & \text { Introduction } & 7\end{array}$

Chapter 2. Continuous passive motion following primary 15 total knee arthroplasty:

Short- and long-term effects on range of motion A systematic review

Chapter 3. Continuous passive motion (CPM) in

rehabilitation following total knee arthroplasty:

a randomised controlled trial

Chapter 4. Reproducibility of goniometric measurement of the knee in the in-hospital phase following total knee arthroplasty.

Chapter 5. Efficiency of immediate postoperative inpatient physiotherapy following total knee arthroplasty: an RCT

Chapter 6. Effectiveness of prolonged use of continuous passive motion (CPM) as an adjunct to physiotherapy following total knee arthroplasty: Design of a randomised controlled trial

Chapter 7. Effectiveness of prolonged use of continuous passive motion (CPM), as an adjunct to physiotherapy, after total knee arthroplasty.

Chapter 8. General discussion

Summary

Samenvatting

Dankwoord

Curriculum vitae

Publications 

Introduction

Aspects of physiotherapy in the peri-operative management of total knee arthroplasty patients 


\section{Introduction}

Knee osteoarthritis $(\mathrm{OA})$ is a prevalent musculoskeletal condition affecting older people, causing pain, physical disability and reduced quality of life $^{1}$. Relevant therapeutic approaches include drug therapy, weight loss, patient education programmes, surgery, walking/household aids or adaptations, and therapeutic exercise ${ }^{2}$.

Total knee arthroplasty (TKA) is considered the treatment of choice for patients with intractable pain and substantial functional disabilities who have not had acceptable relief and functional improvement after conservative treatment ${ }^{3-5}$. Modern TKA began about 1970 . Nowadays, TKA represents a very successful surgical intervention, with reports documenting 15-year survivorship of over $90 \% 6$. Worldwide, 500,000 to 800,000 total knee replacements are performed annually 78 .

Although there is general agreement that exercising after TKA promotes walking ability and independence in activities of daily living, the relative advantages of peri-operative rehabilitation programmes in this population have not been extensively documented. Nevertheless, physiotherapy (PT) is recommended by the American College of Rheumatology and the European League Against Rheumatism in the peri-operative care of knee $\mathrm{OA}^{4}$. It encompasses numerous interventions including exercise, manual techniques, knee taping and education to implement patient self-management strategies.

PT is a therapeutic option in the treatment of OA and the impending interventions in the peri-operative care in knee OA can be related to different phases of the disease. If OA develops and surgery is inevitable, there may be a role for PT in the preoperative instructions of patients. After surgery, patients are seen by physical therapists in the clinical phase, as well as later on in the post clinical rehabilitation phase.

Studies of the effects of PT interventions are difficult to interpret because most studies have not evaluated integral PT programmes but individual components, which do not really reflect typical normal clinical practice.

This introduction presents an overview of the role of PT in the various peri-operative phases, and positions the different components of the thesis in the context of this overview.

\section{Physiotherapy for patients with osteoartritis}

People with symptomatic OA of the knee usually complain of pain and loss of function 910 . In the early stages of the disease, pain is intermittent and mostly associated with joint use (or overuse) ${ }^{10}$. As OA progresses, pain may also be present at rest and during the night. Another accompanying symptom is stiffness, resulting in difficulty of movement especially after a period of rest.

People with progressive symptomatic OA of the knee experience increasing difficulties in daily functional activities. Ultimately, chronic 
OA of the knee leads to reduced physical fitness and limitations in activities of daily living ${ }^{10}$.

International guidelines advocate non-pharmacological treatment as the first line of management for people with $\mathrm{OA}^{4}$.

Exercise therapy may be the PT intervention of choice. Exercise can be divided into joint-specific strength and range of motion exercises and general aerobic conditioning. Furthermore, it can be either directly supervised on land or water or offered as a home-based, self-directed programme 411 .

Next to exercises, a PT programme will encompass education and information on OA and advice on coping strategies 12412 .

A Cochrane review 10 on the effects of therapeutic exercise has demonstrated a beneficial effect of exercises on pain and physical function for people with symptomatic knee OA. Land-based therapeutic exercise was shown to reduce pain and improve physical function. There are, however, insufficient data to provide useful guidelines on optimal exercise type or dosage ${ }^{12}$. Supervised exercise classes appear to be as beneficial as treatment provided on a one-to-one basis [3]. Both high intensity and low intensity aerobic exercises improve functional status, pain, gait and aerobic capacity in people with OA of the knee ${ }^{13}$ No studies have clearly defined the effect of providing information and advice.

Transcutaneous electrical nerve stimulation (TENS) is a modality that is used in PT for the control of pain ${ }^{14}$. A limited number of studies have reported that TENS may be effective for pain control and reduction of stiffness prevalence in OA. However, more data are needed to soundly conclude that TENS is effective in the treatment of OA ${ }^{14}$.

\section{Preoperative exercise and education}

When conservative treatment fails to provide relief in osteoarthritis, knee replacement surgery may be necessary. It is speculated that people who receive pre operative education and exercise and have already been educated about surgery and recovery will be less anxious, can tolerate the pain better and have a shorter hospital stay 15 .

Unfortunately, there is little evidence to support the use of preoperative education over and above standard care to improve postoperative outcomes in patients undergoing knee replacement surgery, especially in terms of pain, functionality and length of hospital stay 15-18. On the other hand, there is evidence that preoperative education has a modest beneficial effect on preoperative anxiety 1519 . However, when preoperative education is tailored to anxiety or targeted at those patients in need of support (e.g. those who are particularly disabled, or have limited social support structures ${ }^{15}$ ) the effect is higher. 


\section{Physiotherapy during hospital stay}

Recent trends in health care encourage early patient discharge from hospitals and substitution of inpatient care with home-based alternatives whenever they are feasible. PT is a component of every in-hospital protocol. Most protocols are similar and incorporate giving information on the surgery and postoperative recovery. Treatment aims to improve range of motion, muscle strengthening (particularly of the quadriceps muscles) and training activities of daily living, like transferring from bed to chair, sitting to standing, walking and climbing stairs $20-22$.

Studies on the effects of PT programmes during this short in-hospital period are lacking. However, there are some reports on the effect of different components of the PT programme. Most research focuses on the application of Continuous Passive Motion (CPM) postoperatively on knee joint mobility. Studies on CPM have produced conflicting results. Some authors postulate that CPM has a positive short term effect on early postoperative knee RoM ${ }^{23-29}$, whereas other studies have found no difference in RoM ${ }^{30-37}$. Furthermore, reports are often unclear about intervention parameters like the range of motion that should initially be utilized, the total treatment duration and the intensity of application.

Four chapters of this thesis focus on the role of PT in the in-hospital period after TKA. Chapter 2 describes a systematic review of randomized controlled trials published between 1966 and July 2002 on the effectiveness of CPM use in restoring early knee RoM in patients after TKA. Chapter 3 presents the design and results of a randomized controlled trial on the effectiveness of short-term use of CPM in patients in the inhospital period after TKA. Results of a study on the reliability of goniometric measurements of the knee in patients after TKA are presented in chapter 4. Chapter 5 presents a study on the efficacy of the overall physiotherapy treatment in the clinical phase after TKA.

Rehabilitation after the immediate postoperative in-patient period Exercise may produce long-term benefits in terms of physical function in patients after TKA. Improvement in strength, balance and other neuromuscular aspects facilitates functional activities and might prevent adverse outcomes such as falls. However, the evidence on exercise as an effective means of rehabilitation after joint replacement is limited, and few controlled trials are available 18 38-41. Although exercise, administered as home programmes or in organized groups led by a physical therapist, has been shown to improve outcomes related to physical activity after total joint arthroplasty, no differences in pain, activities of daily living, quality of life or gait parameters have been demonstrated when comparing organized group exercises with home exercise programmes $39{ }^{40}$. One study, which compared PT with CPM treatment after hospital discharge, found no differences between these two treatment strategies.

Two chapters in this thesis focus on the effect of CPM treatment in this rehabilitation stage. 
Chapter 6 outlines the research design of a study on the effects on range of motion and functional status of prolonged use of a CPM device at home in addition to physiotherapy, compared to physiotherapy alone, in patients with limited flexion range of motion (less than $80^{\circ}$ ) of the knee at discharge from the hospital following TKA. Chapter 7 presents the results of a study on prolonged CPM use.

Finally, chapter 8 reviews the methodology and results of the studies, discusses the implications of findings for contemporary clinical practice and offers recommendations for future research. 


\section{References}

1. Bennell KL, Hinman RS, Metcalf BR, Buchbinder R, McConnell J, McColl $\mathrm{G}$, et al. Efficacy of physiotherapy management of knee joint osteoarthritis: a randomised, double blind, placebo controlled trial. Ann Rheum Dis 2005;64(6):906-12.

2. van Baar ME, Dekker J, Oostendorp RA, Bijl D, Voorn TB, Lemmens JA, et al. The effectiveness of exercise therapy in patients with osteoarthritis of the hip or knee: a randomized clinical trial. $J$ Rheumatol 1998;25(12):2432-9.

3. Brady OH, Masri BA, Garbuz DS, Duncan CP. Rheumatology: 10. Joint replacement of the hip and knee--when to refer and what to expect. Cmaj 2000;163(10):1285-91.

4. Jordan KM, Arden NK, Doherty M, Bannwarth B, Bijlsma JW, Dieppe P, et al. EULAR Recommendations 2003: an evidence based approach to the management of knee osteoarthritis: Report of a Task Force of the Standing Committee for International Clinical Studies Including Therapeutic Trials (ESCISIT). Ann Rheum Dis 2003;62(12):1145-55.

5. Ethgen O, Bruyere O, Richy F, Dardennes C, Reginster JY. Health-related quality of life in total hip and total knee arthroplasty. A qualitative and systematic review of the literature. J Bone Joint Surg Am 2004;86A(5):963-74.

6. Insall JN, Scott W, Scuderi GR. Current concepts in primary and revision total knee arthroplasty. Philidelphia: Lippincot-Raven, 1996.

7. Healy WL, Iorio R, Ko J, Appleby D, Lemos DW. Impact of cost reduction programs on short-term patient outcome and hospital cost of total knee arthroplasty. J Bone Joint Surg Am 2002;84-A(3):348-53.

8. Knutsson S, Engberg IB. An evaluation of patients' quality of life before, 6 weeks and 6 months after total hip replacement surgery. $J$ Adv Nurs 1999;30(6):1349-59.

9. Fautrel B, Hilliquin P, Rozenberg S, Allaert FA, Coste P, Leclerc A, et al. Impact of osteoarthritis: results of a nationwide survey of 10,000 patients consulting for OA. Joint Bone Spine 2005;72(3):235-40.

10. Fransen M, McConnell S, Bell M. Exercise for osteoarthritis of the hip or knee. Cochrane Database Syst Rev 2003(3):CD004286.

11. Bischoff HA, Roos EM. Effectiveness and safety of strengthening, aerobic, and coordination exercises for patients with osteoarthritis. Curr Opin Rheumatol 2003;15(2):141-4.

12. van Baar ME, Assendelft WJ, Dekker J, Oostendorp RA, Bijlsma JW. Effectiveness of exercise therapy in patients with osteoarthritis of the hip or knee: a systematic review of randomized clinical trials. Arthritis Rheum 1999;42(7):1361-9.

13. Brosseau L, MacLeay L, Robinson V, Wells G, Tugwell P. Intensity of exercise for the treatment of osteoarthritis. Cochrane Database Syst Rev 2003(2):CD004259.

14. Osiri M, Welch V, Brosseau L, Shea B, McGowan J, Tugwell P, et al. Transcutaneous electrical nerve stimulation for knee osteoarthritis. Cochrane Database Syst Rev 2000(4):CD002823.

15. McDonald S, Hetrick S, Green S. Pre-operative education for hip or knee replacement. Cochrane Database Syst Rev 2004(1):CD003526. 
16. Mitchell C, Walker J, Walters S, Morgan AB, Binns T, Mathers N. Costs and effectiveness of pre- and post-operative home physiotherapy for total knee replacement: randomized controlled trial. Journal of Evaluation in Clinical Practice 2005;11(3):283-292.

17. Beaupre LA, Lier D, Davies DM, Johnston DB. The effect of a preoperative exercise and education program on functional recovery, health related quality of life, and health service utilization following primary total knee arthroplasty. J Rheumatol 2004;31(6):1166-73.

18. Weaver FM, Hughes SL, Almagor O, Wixson R, Manheim L, Fulton B, et al. Comparison of two home care protocols for total joint replacement. $J$ Am Geriatr Soc 2003;51(4):523-8.

19. Butler GS, Hurley CA, Buchanan KL, Smith-VanHorne J. Prehospital education: effectiveness with total hip replacement surgery patients. Patient Educ Couns 1996;29(2):189-97.

20. Kolisek FR, Gilmore KJ, Peterson EK. Slide and flex, tighten, extend (SAFTE): a safe, convenient, effective, and no-cost approach to rehabilitation after total knee arthroplasty. $J$ Arthroplasty 2000;15(8):1013-6.

21. Roos EM. Effectiveness and practice variation of rehabilitation after joint replacement. Curr Opin Rheumatol 2003;15(2):160-2.

22. Isaac D, Falode T, Liu P, I'Anson H, Dillow K, Gill P. Accelerated rehabilitation after total knee replacement. Knee 2005;12(5):346-50.

23. Pope RO, Corcoran S, Mc Caul K, Howie DW. Continuous Passive Motion after Primary Total Knee Arthroplasty. Does it offer any benefits. Journal of Bone and Joint Surgery 1997;79-B(6):914-917.

24. Vince K, Kelly MA, Beck J, Insall JN. Continuous Passive Motion after Total Knee Arthroplasty. Journal of Arthroplasty 1987;2(4):281-284.

25. Johnson DP, Eastwood DM. Beneficial Effects of Continuous Passive Motion after Total Condylar Knee Arthroplasty. Annals of the Royal College of Surgeons of England 1992;74:412-416.

26. Mc Innes J, Larson MG, Daltroy LH, Brown T, Fossel AH, Eaton HM, et al. A Controlled Evaluation of Patients Undergoing Total Knee Arthroplasty. JAMA 1992;268(11, SEPTEMBER 16):1423-1428.

27. Harms M, Engstrom B. Continuous Passive Motion as an Adjunct to Treatment in the Physical Therapy Management of the Total Knee Arthroplasty Patient. Physiotherapy 1991;77(4, APRIL 1991):301-307.

28. Montgomery F, Eliasson M. Continuous Passive Motion Compared to Active Physical Therapy after Knee Arthroplasty. Acta Orthopedica Scandinavia 1996;67(1):7-9.

29. Beaupré LA, Davies DM, Jones CA, Cinats JC. Exercise Combined With Continuous Passive Motion or Slider Board Therapy Compared With Exercise Only: A Randomized Controlled Trial of Patients Following Total Knee Arthroplasty. Physical Therapy 2001;81(number 4):1029-1037.

30. Kumar PJ, Mc Pherson EJ, Dorr LD, Wan Z, Baldwin K. Rehabilitation After Total Knee Arthroplasty. A Comparison of 2 Rehabilitation Techniques. Clinical Orthopedics and Related Research 1996(331):93-101.

31. Chiarello CM, Gunderson L, O'Halloran T. The Effect of Continuous Passive Motion Duration and Increment on Range of Motion in Total Knee Arthroplasty Patients. JOSPT 1997;25(2):119-127.

32. Worland RL, Arredondo J, Angles F, Jimenez FL, Jessup DE. Home Continuous Passive Motion Machine versus Professional Physical Therapy Following Total Knee Replacement. Journal of Arthroplasty 1998;13(7):784788. 
33. Nielsen PT, Rechnagel K, Nielsen S. No effects ofcontinuous passive motion after arthroplasty of the knee. Acta Orthopedica Scandinavia 1988;59(5):580-581.

34. Colwell CW, Morris BA. The Influence of Continuous Passive Motion on the Results of Total Knee Arthroplasty. Clinical Orthopedics and Related Research 1992(276):225-228.

35. Chen B, Zimmerman JR, Soulen L, DeLisa JA. Continuous Passive Motion after Total Knee Arthroplasty. American Journal of Physical Medicine and Rehabilitation 2000;79(5):421-426.

36. May LA, Busse W, Zayac D, Whitridge MR. Comparison of Continuous Passive Motion Machines and Lower Limb Mobility Boards in the Rehabilitation of Patients with Total Knee Arthroplasty. Canadian Journal of Rehabilitation 1999;12(4):257-263.

37. Walker RH, Morris BAM, Angulo DL, Schneider J, Colwell CW. Postoperative Use of Continuous Passive Motion, Transcutaneous Electrical Nerve Stimulation, and Continuous Cooling Pad Following Total KNee Arthroplasty. The Journal fo Arthroplasty 1991;6(2):151-156.

38. Sashika H, Matsuba Y, Watanabe Y. Home program of physical therapy: effect on disabilities of patients with total hip arthroplasty. Arch Phys Med Rehabil 1996;77(3):273-7.

39. Kramer JF, Speechley M, Bourne R, Rorabeck C, Vaz M. Comparison of clinic- and home-based rehabilitation programs after total knee arthroplasty. Clin Orthop Relat Res 2003(410):225-34.

40. Rajan RA, Pack Y, Jackson H, Gillies C, Asirvatham R. No need for outpatient physiotherapy following total knee arthroplasty: a randomized trial of 120 patients. Acta Orthop Scand 2004;75(1):71-3.

41. Worland RL, Arredondo J, Angles F, Lopez-Jimenez F, Jessup DE. Home continuous passive motion machine versus professional physical therapy following total knee replacement. J Arthroplasty 1998;13(7):784-7. 
Continuous passive motion following primary total knee arthroplasty:

Short- and long-term effects on range of motion A systematic review

AF Lenssen

AJA Köke

RA de Bie

RJT Geesink

Physical Therapy reviews 2003, 8:113-121. 


\section{Abstract}

\section{Study design}

a systematic review of randomized controlled trials

\section{Background}

Knee arthroplasties are a common surgical procedure for patients suffering from knee osteoarthritis. Continuous passive motion (CPM) is frequently used to increase range of motion (RoM) in the knee and promote rapid postoperative recovery of patients undergoing total knee arthroplasty (TKA). Many clinical trials have tried to assess its efficacy, but results have been contradictory.

\section{Objectives}

To review the efficacy in terms of RoM of CPM in the postoperative management of patients undergoing TKA.

\section{Methods}

Medline, Embase, Cinahl and Cochrane databases and bibliographic indexes and relevant citations were searched. All relevant studies were assessed for methodological quality using a validated scoring instrument.

\section{Data collection \& analysis}

Two reviewers independently extracted data and assessed trial quality. Results of a meta-analysis of RoM data were divided into short-term effects, i.e., seven to fourteen days after surgery, and long-term effects, i.e., six to twelve months after surgery. Where possible, data from individual trials were combined in a meta-analysis. Data were analysed using weighted mean differences (WMD) with 95\% confidence intervals (CI) between treatment and control groups in the short and long term, weight being the inverse of variance.

\section{Main results}

Fifteen studies investigating the effect of continuous passive motion in the management of TKA patients were included in the review. Study quality ranged from poor ( 2 points on a ten-point scale) to good (8 points).

The evidence on the efficacy of CPM after total knee arthroplasty is conflicting. Positive short-term effects of adding CPM to standardised physiotherapy (PT) have been reported on flexion RoM. Five studies were included in a pooled analysis, for a total of 317 patients (pooled effect size WMD $\left.8.27^{\circ}, 95 \% \mathrm{CI}-1.60,18.15\right)$

\section{Reviewers' conclusions}

There is evidence of a moderate positive, short-term effect of adding CPM to standardised PT after total knee arthroplasty.

There is no relevant long-term effect of CPM use. 


\section{Background}

Total knee arthroplasty (TKA) is a common form of orthopaedic surgery, mostly in osteoarthritis patients. Since patients undergoing joint arthroplasty are discharged from the hospital within 1-2 weeks after the operation, the main targets of rehabilitation are rapid mobilisation of the patient and early regaining of the range of motion (RoM) in the knee. RoM exercises start on the day of surgery, and most rehabilitation programmes describe mobilisation of the patients within the first few days after surgery ${ }^{1-6}$.

Continuous passive motion (CPM) is a motorized device, which enables the knee joint to move throughout an arc of range of motion. It has been used in the rehabilitation of patients after knee surgery ever since Salter and colleagues introduced the device in the early 1980s ${ }^{7}$. Beneficial effects that have been claimed include relative freedom of pain, maintenance of range of motion, improved wound healing and quicker recovery. Although CPM is widely used, the debate about its efficacy is still ongoing, mainly as a result of controversial findings reported in the literature. The first randomized controlled trials date from the end of the 1980 s, some ten years after the introduction of CPM as a treatment modality. Some trials have shown positive effects on early postoperative knee RoM 1-68, whereas other studies have found no difference in RoM 916. Nearly all authors concluded that the use of CPM did not result in better RoM 3-6 months after the operation. This review focuses on flexion RoM because gain of flexion RoM is the prime reason for adding $\mathrm{CPM}$ in a rehabilitation program following TKA. Knee flexion is important in mobility tasks like walking, sit-to-stand transfers and other activities of daily living.

The present systematic review was undertaken to summarise the evidence on efficacy of CPM use after TKA and to gain insight into the methodological quality of previously performed studies. Specifically, the first question of this review is whether CPM is more effective as a method for early restoration of knee RoM (7-14 days after surgery) in patients following TKA than comparable treatments. The second question is if the effect of CPM use on the long-term knee RoM holds.

\section{Materials and Methods}

\section{Inclusion criteria for the review}

The review included only randomised trials (RCTs) involving patients who had undergone primary TKA, using CPM as one of the treatments under investigation and measuring outcome by range of motion (RoM). Only RCTs published in English, Dutch and German were included. 


\section{Search strategy to identify studies}

All relevant RCTs meeting the inclusion criteria were identified by a computer-aided search of the MEDLINE (1966 to July 2002) the Cochrane Controlled Trials Register, Cinahl and EMBASE (1988 to July 2002) databases, using the following search terms: 'continuous passive motion', 'CPM', 'total knee', 'therapy', and 'exercise'. Additionally, references given in relevant identified publications were screened.

\section{Methods of the review}

Description of studies

The first author selected RCTs in which CPM was one of the treatments under investigation and either compared CPM to no treatment or to physiotherapy without CPM.

\section{Methodological quality of studies}

The methodological quality of the RCTs up to July 2002 was independently assessed by two reviewers (AJA Köke, RA de Bie), using the Maastricht Amsterdam Consensus List (table 1) ${ }^{17}$. Only criteria reflecting the internal validity of the RCTs were scored. Disagreements between the two reviewers were solved by consensus after a third reviewer (AF Lenssen) had been consulted. Each criterion was scored as 'positive', 'negative' or 'unclear'. A total score was computed by counting the numbers of positive scores.

The findings were summarised by means of meta-analysis if there were more than three studies that could be pooled (figure 1). Otherwise a rating system was used for levels of evidence, consisting of four levels of scientific evidence ${ }^{17}$ :

1. Strong evidence - provided by generally consistent findings in multiple high-quality RCTs.

2. Moderate evidence - provided by generally consistent findings in one high-quality RCT and one or more low-quality RCTs or by generally consistent findings in multiple low-quality RCTs.

3. Limited or conflicting evidence - only one RCT available (either high or low quality) or inconsistent findings in multiple RCTs.

4. No evidence - no RCTs. 
We defined high-quality studies as RCTs which met four or more of the validity criteria.

Table 1. Maastricht Amsterdam Consensus List (methodological criteria list) ${ }^{17}$

1. Method of randomisation

2. Similarity of baseline characteristics

3. Blinding of care provider

4. Co-interventions avoided or equal

5. Compliance

6. Blinding of patients

7. Blinding of outcome assessment

8. Drop-out rate/loss to follow-up

9. Adequate length of follow-up

10. Intention-to-treat analysis

$\begin{array}{lll}+ & - & ? \\ + & - & ? \\ + & - & ? \\ + & - & ? \\ + & - & ? \\ + & - & ? \\ + & - & ? \\ + & - & ? \\ + & - & ? \\ + & - & ?\end{array}$

Legend: a '+' results in 1 point, a '-' or question mark denotes no points

The present review focuses on the effects on RoM, more specifically on flexion RoM.

Outcome assessment was divided into short-term (5-14 days after surgery) and long-term

(3-6 months after surgery).

\section{Results}

\section{Study selection}

Our primary search resulted in 23 references from Medline, Embase, Cochrane and Cinahl. In the end, however, only 15 articles were included, for the following reasons. Two studies 1819 compared different forms of CPM use. One study turned out not to be a randomised controlled trial after all ${ }^{20}$, whereas in another study ${ }^{21}$ only patients with bilateral knee arthroplasty were randomised, leaving it unclear whether a random assignment had also been performed on patients with unilateral arthroplasty. One study used CPM as a baseline treatment for all treatment groups and not as an experimental intervention 22 , while a further study 23 included only patients with bilateral arthroplasty. In another study 24 18-24 hours of CPM use on the first day after surgery was compared with no CPM use, with effects being measured at six and 52 weeks. This study was excluded due to the short duration of CPM use relative to the timing of the follow-up measurements. Finally, one article was written in Polish ${ }^{25}$, and since the authors of this review are unable to read papers in this language, this article was not included either. 


\section{Methodological quality}

Table 2 shows the final results of the quality assessment. The methodological quality ranged from 2 to 8 points.

Table 2: methodological scores of publications reviewed

$1^{+}=$positive, - = negative, ? = unclear), with numbers of positive (" 1 ") scores. 'Informativity' was calculated by dividing the number of items with a positive or negative score by the total number of items and expressing this as a percentage.

\begin{tabular}{|c|c|c|c|c|c|c|c|c|c|c|c|c|c|}
\hline$\overline{\text { Study }}$ & & 1 & 2 & 3 & 4 & 5 & 6 & 7 & 8 & 9 & 10 & $\begin{array}{c}\text { No. of } \\
\text { " } 1 \text { " } \\
\text { scores }\end{array}$ & $\begin{array}{c}\text { Informa- } \\
\text { tivity }\end{array}$ \\
\hline Beaupré ${ }^{2}$ & 2001 & 1 & 1 & - & 1 & 1 & - & 1 & 1 & 1 & 1 & 8 & $100 \%$ \\
\hline Mc Innes ${ }^{3}$ & 1992 & 1 & 1 & - & 1 & ? & - & 1 & 1 & 1 & - & 6 & $90 \%$ \\
\hline Kumar $^{9}$ & 1996 & 1 & 1 & - & 1 & ? & - & 1 & 1 & 1 & - & 6 & $90 \%$ \\
\hline Worland 11 & 1998 & 1 & 1 & - & ? & ? & - & 1 & 1 & 1 & 1 & 6 & $80 \%$ \\
\hline Nielsen 12 & 1988 & 1 & 1 & - & - & - & - & 1 & 1 & 1 & & 5 & $80 \%$ \\
\hline Colwell 13 & 1992 & 1 & 1 & - & 1 & ? & - & ? & 1 & 1 & - & 5 & $80 \%$ \\
\hline Chen 14 & 2000 & 1 & 1 & - & ? & 1 & - & 1 & - & 1 & ? & 5 & $80 \%$ \\
\hline Vince 6 & 1987 & 1 & 1 & - & 1 & ? & - & ? & $?$ & 1 & $?$ & 4 & $60 \%$ \\
\hline Johnson 5 & 1992 & 1 & $?$ & - & ? & ? & - & - & 1 & 1 & 1 & 4 & $70 \%$ \\
\hline Montgomery $^{8}$ & 1996 & 1 & 1 & - & ? & ? & - & ? & 1 & 1 & ? & 4 & $60 \%$ \\
\hline Pope ${ }^{4}$ & 1997 & 1 & 1 & - & ? & ? & - & ? & 1 & 1 & - & 4 & $70 \%$ \\
\hline Chiarello 10 & 1997 & 1 & 1 & - & ? & 1 & - & - & 1 & ? & - & 4 & $80 \%$ \\
\hline Harms 1 & 1991 & 1 & 1 & - & - & ? & - & ? & ? & 1 & ? & 3 & $60 \%$ \\
\hline May 15 & 1999 & 1 & - & - & ? & ? & - & 1 & $?$ & 1 & ? & 3 & $60 \%$ \\
\hline Walker 16 & 1991 & 1 & ? & ? & ? & ? & - & ? & - & 1 & - & 2 & $50 \%$ \\
\hline
\end{tabular}

Comparability at baseline, dropout rate and timing of the effect measurement were described in most articles. Blinding of patients and therapist is extremely difficult given the type of treatment involved, and had been applied in none of the studies. Blinding of outcome assessment was unclear in six studies 1468813 16. Compliance (actual time spent in the CPM machine) was only measured in three studies.

Chen et al. ${ }^{14}$ reported on 64 randomised patients, of whom thirteen decided not to participate in the trial after all. These patients were excluded from further analysis in the trial, a deviation from the intention-to-treat principle that might lead to serious bias.

Harms and Engstrom ${ }^{1}$ reported co-interventions divided unequally over the intervention and control groups. Five patients in the non-CPM group needed manipulation under anaesthesia, compared to one in the CPM group. Finally, intention-to-treat analysis was only performed in three of the fourteen studies 2511 . 


\section{Study characteristics}

Study characteristics are presented in table 3. Nine studies $13461012-14$ 16 were identified that compared standardised PT plus CPM with a control group that received only the standardised rehabilitation programme. Two studies 811 compared CPM with a physiotherapy programme. One study ${ }^{5}$ compared CPM with cast immobilisation. Two studies ${ }^{2} 15$ compared CPM with the use of a slider board. Finally, in one study ${ }^{9}$ CPM plus Standard PT was compared with PT plus a "drop and dangle" regimen.

\section{Effect measurement}

Although RoM measurement was a primary outcome in all publications, various studies used different methods to measure RoM; active, passive total RoM or only active flexion RoM (Table 3).

\section{Effects on RoM}

Although many outcome measures were used in the fourteen studies, only RoM was used as such in all. The present review focuses on the effects on RoM, more specifically on flexion RoM. We divided outcome assessment into short-term (7-14 days after surgery) and long-term (612 months) assessments.

\section{Effects of CPM on flexion RoM}

CPM + Physiotherapy vs Physiotherapy

Nine studies 134610 12-14 16 were identified that compared standardised PT plus CPM with a control group that received only the standardised rehabilitation programme. Two studies 1316 did not report a short-term measurement, so these were not included in the meta-analysis of shortterm effects. Two studies 612 could not be included in the meta-analysis, because they failed to present the necessary data. This left five studies ${ }^{1}$ 341014 to be included in a meta-analysis of short-term effects on flexion RoM of CPM treatment plus PT versus PT alone. One ${ }^{3}$ of these five studies did not report SD but did report P-values. SD values for this study were estimated from the pooled SD (computed with means, sample sizes and P-values). Two studies 110 reported outcomes as graphs, the mean scores and SDs were estimated from the graphs. Using the Random effects model, the pooled weighted mean difference (WMD) post treatment for CPM added to PT vs PT was 8.27 (95\% CI -1.60, 18.15), showing moderate but conflicting evidence that adding CPM to standardised PT treatment is effective in restoring flexion RoM more rapidly. 


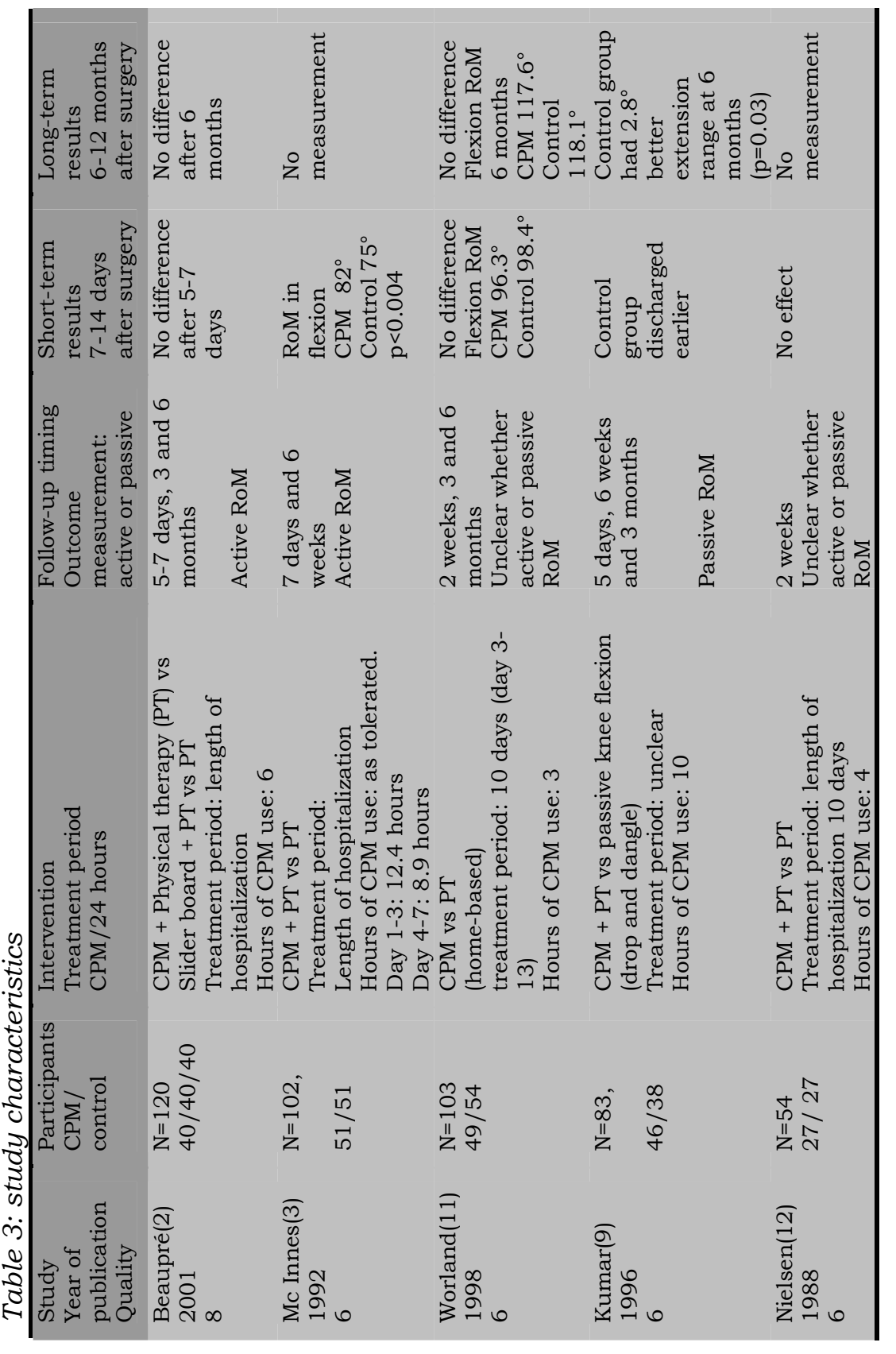




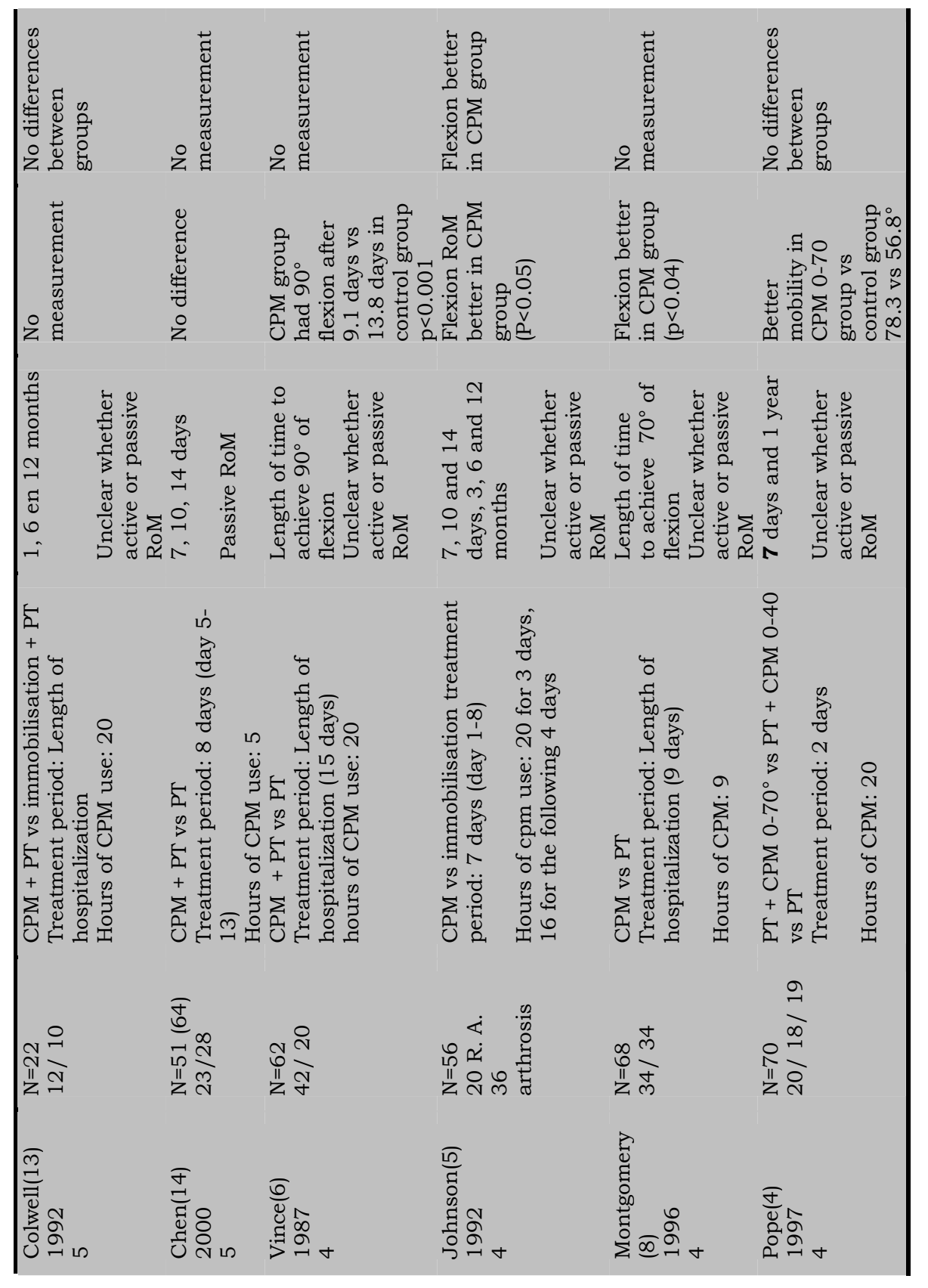




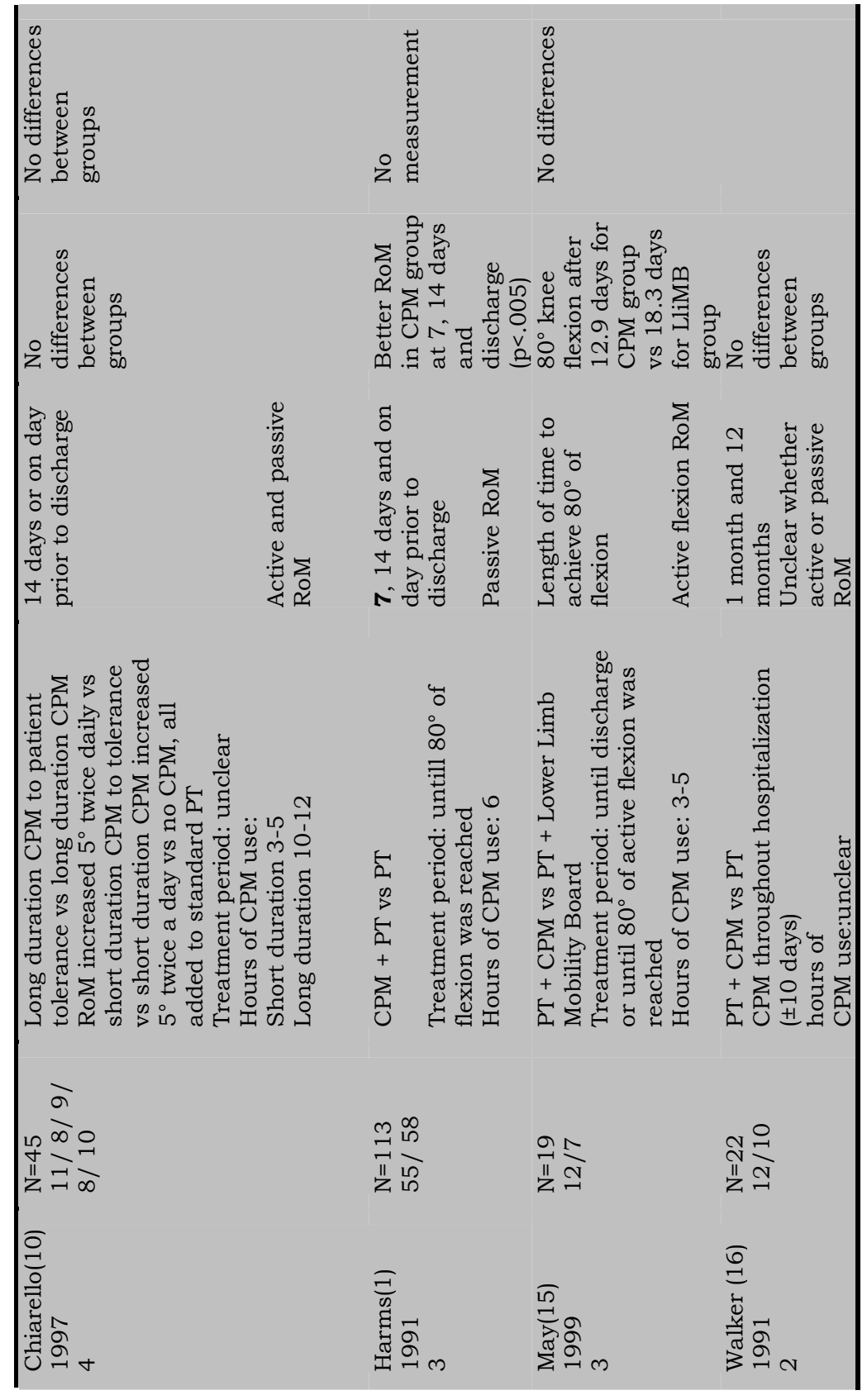


Only four studies 241316 reported long-term results; these reported no statistically significant difference between the groups. Only Pope et al. 4 and Colwell et al. 13 provided necessary data for meta-analysis but the contrast between CPM and control group differed in these studies. Although there was a slight but significant difference between the treatment and control groups ( $2^{\circ}$ after 6 months) we believe that this difference is not clinically relevant. There is moderate evidence that CPM treatment in addition to standardised PT is no more effective in improving long-term flexion RoM than standardised PT alone.

Figure 1: meta analysis of comparison $C P M+P T$ vs $P T$

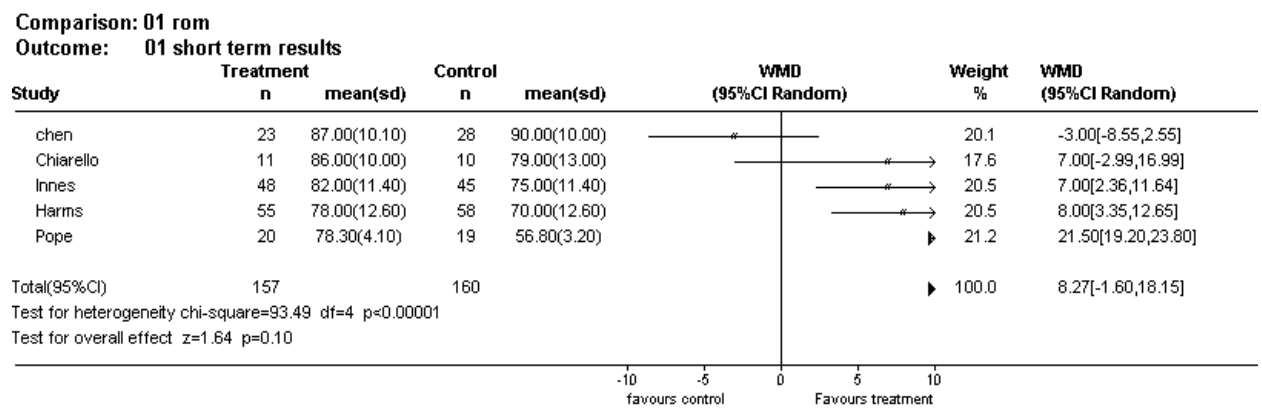

\section{CPM vs PT}

Two studies 811 compared CPM with a physiotherapy programme. Montgomery et al. ${ }^{26}$ reported short-term effects of CPM relative to PT, while Worland et al. ${ }^{27}$ reported no differences between CPM and PT. Only Worland et al. ${ }^{27}$ reported long-term results, finding no difference between the two approaches.

There was conflicting evidence concerning the short-term efficacy of CPM use when compared to PT. There was limited evidence that CPM is no more effective than PT in the long-term following total knee arthroplasty.

\section{CPM vs immobilisation}

One study compared CPM with seven days of cast immobilisation. Both short- and long-term results favoured CPM treatment over immobilisation (mean flexion RoM at seven days after surgery was $68^{\circ}$ in the CPM group vs $51^{\circ}$ in the control group). At six months, Johnson et al. (4) still reported a mean deficit of $10^{\circ}$ in the immobilisation regimen group. There was limited short- and long-term evidence in favour of CPM treatment when compared to immobilisation in the first week after the operation. 


\section{CPM vs slider board}

Two studies 215 compared CPM with the use of a slider board, comparing standardised exercises (SE) plus CPM with SE plus slider board treatment. Both reported no short- or long-term differences between the two strategies. There was limited evidence from the comparison between CPM and slider board treatment, with both regimens equally successful in restoring RoM.

\section{CPM + PT vs Pt + "drop and dangle"}

Kumar et al. ${ }^{9}$ compared CPM plus Standard PT with PT plus a "drop and dangle" regimen. Patients in the "drop and dangle" group remained with the knee in maximum flexion for a minimum of 20 minutes twice a day. The authors reported no difference 5 days after surgery but slightly better flexion RoM in the CPM group (average $2.5^{\circ}$ ) after 3 months.

\section{Discussion}

The present systematic review included 15 studies, and showed that the evidence on the efficacy of CPM after TKA is conflicting. Positive shortterm effects of adding CPM to standardised physiotherapy (PT) have been reported (pooled effect size WMD 8.27, $95 \%$ CI $-1.60,18.15 \mathrm{n}=5$ studies). A minor difference in favour of adding CPM to standard PT has been found at 6-12 months after operation (weighted mean difference $1.98^{\circ}, 95 \%$ CI $0.21-3.75 ; \mathrm{n}=2$ studies).

There is moderate yet conflicting evidence concerning the short-term efficacy of CPM use when compared to Pt. There is limited evidence for the efficacy of CPM when compared to immobilisation. There is limited evidence that CPM is not more effective when compared to other active regimen, like slider board or drop and dangle treatment.

Studies were heterogeneous in terms of the kind of control interventions used. Methods used to measure RoM also differed between studies, with three using active RoM measurement three passive and one both, while the other eight publications did not state clearly whether active or passive RoM measurements had been used. Not all studies reported short- and long-term results.

We chose to review only RoM as an outcome, because RoM is the primary outcome measure of the effect of CPM after total knee arthroplasty. Moreover, other possible outcome measures, such as pain or functionality, were poorly described in the studies of CPM use.

The methodological quality of the studies varied. It must be remembered, however, that some of the internal validity criteria are very difficult to meet for studies in this field. It is impossible, for instance, to blind patients. The criteria on the blinding of care providers was not met by any of the studies; this is obviously also difficult to achieve. 
Not blinding may lead to bias, because knowledge of the treatment given can influence the results. This makes it all the more important to blind the outcome assessor(s). Only half of the studies, however, used a blinded outcome assessor.

Another aspect of quality assessment was that compliance (the actual amount of time spent in the CPM machine), was only included in the outcome assessment in three studies. Since one believes in a doseresponse relationship between CPM and RoM, it is remarkable that most authors failed to check for this. Finally, no intention-to-treat analysis was performed in eleven of the fourteen studies. However, there does not seem to be a relationship between study quality and outcome for the studies included in the present review.

We performed a meta-analysis only for those studies comparing CPM plus PT with PT alone. We chose to do so because these studies were homogeneous with respect to contrast and timing of effect measurements.

Unfortunately, the presentation of data in individual studies led to the inclusion of only five of the nine possible studies in the meta-analysis. Of these five, we had to assume equal variance in two studies to calculate pooled SDs. However, we believe that a meta-analysis is appropriate for this comparison and provides more insight than merely using levels of evidence to summarize results.

Our analysis show that there is moderate yet conflicting evidence on the short-term efficacy of CPM treatment added to standardised PT in comparison to standardised PT alone. Outcomes of more recent studies appear to differ from those of studies performed in the 1990s. A possible explanation could be that control treatments are more demanding nowadays compared to 10 years ago. This explanation is even more plausible if we take the other comparisons into account.

Studies in which there was considerable contrast between the experimental and control treatments 5 , for instance in CPM versus immobilisation, found major differences in effect. Studies comparing CPM with active and more comparable regimens, like drop and dangle or slider board treatment, did not seem to find clinical differences.

This leads us to conclude that CPM is more effective if the control treatment is passive. The more the control treatment resembles the CPM treatment in terms of the amount of time spent exercising, the smaller the differences that can be expected. Although no long-term effects were reported by the three studies which included long-term measurement, a slight difference in effect was found in favour of CPM treatment. We believe, however, that this minor difference is of no clinical relevance. 


\section{Reviewers' conclusion}

There is moderate yet conflicting evidence of the short-term efficacy of CPM use. When CPM is added to a physiotherapy programme, the additional effect seems to be related to the intensity of the PT programme.

Comparisons with PT have yielded conflicting evidence on the merits of the two treatment modalities. There is no long-term benefit of CPM use.

\section{Implications for practice}

The authors suggest incorporating CPM treatment in inactive patients and if rapid RoM gain is of great importance.

\section{Implications for research}

An important topic for future research could be the identification of relevant subgroups, assessing whether CPM could be more effective in patients that are not able to engage in intensive PT, or in situations where active PT is not available. Another relevant topic for future research could be to assess whether longer use of CPM, for instance for two weeks after surgery, could lead to greater improvement.

A third topic would be the effect of CPM on other outcome measures not included in the present review.

\section{Acknowledgements}

We would like to thank Maurice Zeegers (department of epidemiology, Maastricht University) for his help with the meta-analysis. We would also like to thank all staff of the Physiotherapy Department of the University Hospital Maastricht for giving us the opportunity to write this review. 


\section{References}

1. Harms M, Engstrom B. Continuous Passive Motion as an Adjunct to Treatment in the Physical Therapy Management of the Total Knee Arthroplasty Patient. Physiotherapy 1991;77(4, APRIL 1991):301-307.

2. Beaupré LA, Davies DM, Jones CA, Cinats JC. Exercise Combined With Continuous Passive Motion or Slider Board Therapy Compared With Exercise Only: A Randomized Controlled Trial of Patients Following Total Knee Arthroplasty. Physical Therapy 2001;81(number 4):1029-1037.

3. Mc Innes J, Larson MG, Daltroy LH, Brown T, Fossel AH, Eaton HM, et al. A Controlled Evaluation of Patients Undergoing Total Knee Arthroplasty. JAMA 1992;268(11, SEPTEMBER 16):1423-1428.

4. Pope RO, Corcoran S, Mc Caul K, Howie DW. Continuous Passive Motion after Primary Total Knee Arthroplasty Does it offer any benefits. Journal of Bone and Joint Surgery 1997;79-B(6):914-917.

5. Johnson DP, Eastwood DM. Beneficial Effects of Continuous Passive Motion after Total Condylar Knee Arthroplasty. Annals of the Royal College of Surgeons of England 1992;74:412-416.

6. Vince K, Kelly MA, Beck J, Insall JN. Continuous Passive Motion after Total Knee Arthroplasty. Journal of Arthroplasty 1987;2(4):281-284.

7. Salter RB. The Biologic Concept of Continuous Passive Motion of Synovial Joints. Clinical Orthopedics and Related Research 1989;242(may 1989):12-26.

8. Montgomery F, Eliasson M. Continuous Passive Motion Compared to Active Physical Therapy after Knee Arthroplasty. Acta Orthopedica Scandinavia 1996;67(1):7-9.

9. Kumar PJ, Mc Pherson EJ, Dorr LD, Wan Z, Baldwin K. Rehabilitation After Total Knee Arthroplasty A Comparison of 2 Rehabilitation Techniques. Clinical Orthopedics and Related Research 1996(331):93-101.

10. Chiarello CM, Gunderson L, O'Halloran T. The Effect of Continuous Passive Motion Duration and Increment on Range of Motion in Total Knee Arthroplasty Patients. JOSPT 1997;25(2):119-127.

11. Worland RL, Arredondo J, Angles F, Jimenez FL, Jessup DE. Home Continuous Passive Motion Machine versus Professional Physical Therapy Following Total Knee Replacement. Journal of Arthroplasty 1998;13(7):784788.

12. Nielsen PT, Rechnagel K, Nielsen S. No effects ofcontinuous passive motion after arthroplasty of the knee. Acta Orthopedica Scandinavia 1988;59(5):580-581.

13. Colwell CW, Morris BA. The Influence of Continuous Passive Motion on the Results of Total Knee Arthroplasty. Clinical Orthopedics and Related Research 1992(276):225-228.

14. Chen B, Zimmerman JR, Soulen L, DeLisa JA. Continuous Passive Motion after Total Knee Arthroplasty. American Journal of Physical Medicine and Rehabilitation 2000;79(5):421-426.

15. May LA, Busse W, Zayac D, Whitridge MR. Comparison of Continuous Passive Motion Machines and Lower Limb Mobility Boards in the Rehabilitation of Patients with Total Knee Arthroplasty. Canadian Journal of Rehabilitation 1999;12(4):257-263. 
16. Walker RH, Morris BAM, Angulo DL, Schneider J, Colwell CW. Postoperative Use of Continuous Passive Motion, Transcutaneous Electrical Nerve Stimulation, and Continuous Cooling Pad Following Total KNee Arthroplasty. The Journal fo Arthroplasty 1991;6(2):151-156.

17. van Tulder MW, Assendelft WJJ, Koes BW, Bouter LM. method guideline for systematic reviews in the cochrane collaboration back review group for spinal disorders. spine 1997;22(20):2323-2330.

18. London NJ, Brown M, Newman RJ. Continuous Passive Motion; Evaluation of a New Portable Low Cost Machine. Physiotherapy 1999;85(11):610-612.

19. Yashar AA, Venn-Watson E, Welsh T, Colwell CW, Lotke P. Continuous Passive Motion with Accelerated Flexion after Total Knee Arthroplasty. Clinical Orthopedics and Related Research 1997(345):38-43.

20. Ververeli PA, Sutton DC, Hearn SL, Booth RE, Hozack WJ, Rothman RR. Continuous Passive Motion after Total Knee Arthroplasty. Analyses of cost and benefits. Clinical Orthopedics 1995(321):208-215.

21. Lau SKI, Chiu KY. Use of Continuous Passive Motion after Total Knee Arthroplasty. Journal of Arthroplasty 2001;16(3):336-339.

22. Angulo DL, C.W. C. Use of Postoperative Tens and Continuous Passive Motion following Total Knee Replacement. JOSPT 1990;11(12):599-604.

23. Ritter MA, Gandolf VS, Holston KS. Continuous Passive Motion versus Physical Therapy in Total Knee Arthroplasty. Clinical Orthopedics and Related Research 1989(244):239-243.

24. MacDonald SJ, Bourne RB, Rorabeck CH, McCAlden RW, Kramer J, Vaz M. Prospective Randomized Clinical Trial of Continuous Passive Motion after Total Knee Arthroplasty. Clinical Orthopedics and Related Research 2000(380):30-35.

25. Sosin P, Dutka J, Stabach M. A Comparison of Kinesitherapy With and Without Continuous Passive Motion After the Entire Allograft Surgery of the Knee. Chir Narzadow Ruchu Ortop Pol 2000(65 (1)):47-53.

26. Montgomery F, Eliasson M. Continuous passive motion compared to active physical therapy after knee arthroplasty: similar hospitalization times in a randomized study of 68 patients. Acta Orthop Scand 1996;67(1):7-9.

27. Worland RL, Arredondo J, Angles F, Lopez-Jimenez F, Jessup DE. Home continuous passive motion machine versus professional physical therapy following total knee replacement. J Arthroplasty 1998;13(7):784-7. 


\section{3}

Continuous passive motion (CPM) in rehabilitation following total knee arthroplasty:

a randomised controlled trial

AF Lenssen

RA de Bie

SK Bulstra

MJA van Steyn

Physical Therapy Reviews 2003, 8:123-129. 


\begin{abstract}
Objective

Continuous passive motion (CPM) has been shown to increase the amount of knee flexion for knee patients at the acute care hospital.

Changing postoperative management leads to short hospitalization periods. The objective of the present randomized controlled trial was to assess whether there is additional benefit in CPM use during such a short term hospitalization period.
\end{abstract}

\title{
Design
}

Forty patients undergoing total knee arthroplasty were randomly allocated to either a group receiving CPM in addition to physiotherapy or a group receiving physiotherapy alone.

Both programmes were delivered during a 5-day postoperative period on an inpatient basis, starting on the first day after surgery.

Main outcome measures were mobility and function; secondary measures included muscle strength, pain, satisfaction and length of hospital stay.

\section{Results}

The results indicate a significant difference in function score, pain and strength between the CPM group and the control group. Four days after surgery the CPM group reaches an average of 56 points on the Hospital for Special Surgery Scale (HSS) versus 45 points in the control group $(\mathrm{p}=.005)$.

\section{Conclusions}

The results indicate that in addition to an improved range of motion, a protocol including CPM seems to have a favourable effect on pain and muscle strength in the first two weeks after surgery. 


\section{Introduction}

Early mobilization, if possible starting on the day of surgery, is the strategy of choice in orthopaedic operations in the Netherlands. Continuous Passive Motion (CPM) has been used in the rehabilitation of patients after knee surgery ever since Salter and colleagues ${ }^{1}$ introduced the device in the early 1980s. The beneficial effects ${ }^{12}$ claimed for it include pain relieve, maintenance of range of motion, improved wound healing, and quicker recovery.

The first randomized controlled trials (RCTs) in this field date from the end of the 1980s ${ }^{3-5}$, some 10 years after the introduction of CPM as a treatment modality.

Although CPM is widely used, the debate about its efficacy is still ongoing, mainly as a result of controversial findings reported in the literature.

A systematic review ${ }^{6}$ using an extended Medline search found 15 RCTs 3 5 7-19 addressing CPM use after total knee implantation. There was considerable variety in methodological quality (ranging from poor to good) as well as in the treatments compared. Studies were difficult to compare because they used 18 different outcome measures. Major outcome measures were mobility, which was assessed in all 15 trials, length of hospital stay, assessed in five 511121516 and drain production, assessed in four 8 15-17.

Overall, there seem to be short-term beneficial effects on mobility. Of six studies measuring mobility within the first two weeks after the operation, five reported positive effects of CPM 38 10-12 16. Reported differences between CPM and control groups range from $-3^{\circ}$ to $+22^{\circ}$ in favour of the CPM group. The functional consequences of faster mobility improvement remain unclear, however, and in the long term there do not seem to be advantages of the use of CPM ${ }^{6}$.

Over the last few years, however, treatment protocols following total knee arthroplasty have changed. The formally common fortnight hospital stay has gradually been reduced to 4-5 days. And one week of bed rest has been replaced by mobilization of the patients starting on the first or second day after the operation 8101116 .

These protocol changes raise the question whether the effects on CPM reported in relation to the older protocols are still valid. Rehabilitation now starts on the first day after surgery, resulting in activity during the first two stages of connective tissue repair. It remains unclear what effect intensive exercise and mobilization have on these first phases of tissue repair, whether they shorten or prolong the bleeding and oedema phase, and whether this could influence clinical outcome. To answer these questions, a randomized controlled trial was performed in the university hospital in Maastricht. Physiotherapy (PT) without CPM (control group) was compared with physiotherapy with CPM (CPM group) as an adjunct treatment. 
The study was approved by the medical ethics committee of the University Hospital of Maastricht and Maastricht University.

\section{Patients and methods}

\section{Patients}

All consecutive patients with osteoarthritis undergoing primary total knee arthroplasty (TKA) from January to May 2001 were screened. Patients with relevant co morbidity, such as Rheumatoid arthritis, were excluded, as were patients for whom a longer stay was expected due to co-morbidity. Figure 1 illustrates the design of the study.

Fig 1: study design and outcome assessment.

T0

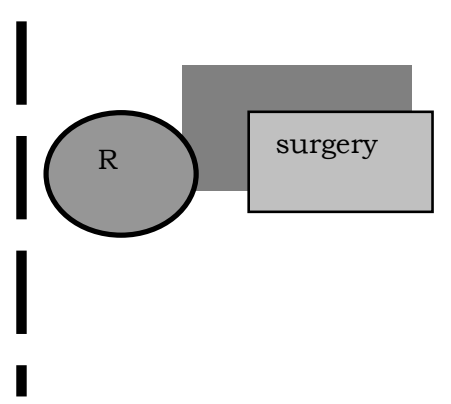

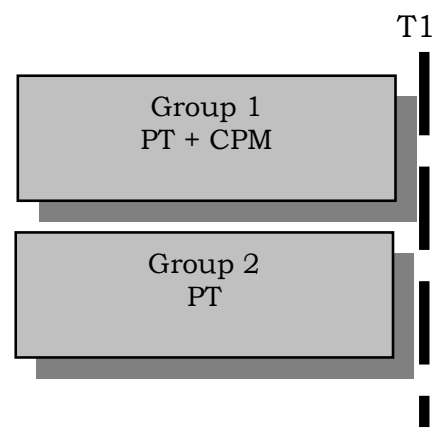

$\mathrm{T} 2$
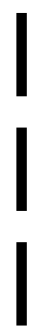

I

TO baseline assessment, one week before surgery,

$\mathrm{T} 1$ assessment 4 days after surgery,

T2 assessment 17 days after surgery,

$\mathrm{R}=$ randomisation

\section{Randomization}

Patients were randomly allocated to one of the treatment groups by means of a computer-generated table, with a block size of eight. An independent orthopaedic secretary without knowledge of the randomization schedule called up the patients for operation. The patients assigned to the CPM group and those included in the control group were operated on in different weeks, to prevent bias caused by patients of both groups being in the hospital at the same time.

\section{Intervention}

On the day of surgery, all patients took bed rest. On the first day after surgery, they began walking. PT was standardised by means of the Maastricht Hospital protocol for in-patient treatment of Total Knee patients. This protocol prescribes hospital discharge on the fourth day after the operation. 
PT was aimed at strengthening the $\mathrm{m}$. quadriceps and mobilizing the knee (actively as well as passively, using joint distraction and angular mobilization techniques). Care was taken to restore a proper gait pattern with the help of one elbow crutch upon discharge. The target for mobility in the clinical phase was $80^{\circ}$ of knee flexion and no more than $10^{\circ}$ of knee extension deficit.

After discharge, all patients received PT treatment at home, three times a week.

The control group received in-hospital PT twice a day for 20 minutes for four days. The CPM group additionally received four hours of CPM daily. The CPM machines used were "Toronto medical" (OrthoLogic 1td. Toronto, Canada) with adjustable upper and lower leg lengths. RoM was increased daily as tolerated.

As a result of the design, the only contrast between the two groups during the hospital stay was the use of CPM in the experimental group. All patients received pain medication in accordance with the hospital protocol, using epidural or I.V. medication for the first 48 hours, followed by oral pain medication during the next 2 days.

\section{Outcome assessment}

All physical measurements were performed by one, independent, blinded observer. Passive RoM was measured using a "long arm" goniometer. Extension was measured with the patient supine, flexion with the patient seated and hips at $90^{\circ}$. Function was evaluated using the "Hospital for Special Surgery" score 20.

Data on medication and length of hospital stay were abstracted from medical records.

Primary outcome measures were mobility and function, both of which were assessed at all three examinations.

Secondary outcome measures included length of hospital stay, pain, muscle strength, medication, satisfaction about the treatment given by the physical therapist, and satisfaction with attention received from the physical therapist. The total amount of time spent in the CPM machine per day was measured to assess compliance.

Muscle strength was tested using a hand-held dynamometer ( $\mathrm{Citec}^{\mathrm{TM}}$, Centre for Innovative Technics, Haren, the Netherlands) with the patient seated, hips at $90^{\circ}$ and knees at $60^{\circ}$ of flexion, using the "make" method. In this method, the patient tries to extend the leg and the observer applies a counter pressure so the knee remains in $60^{\circ}$ of flexion. An 11-point scale was used to rate pain and satisfaction with treatment and attention received from the physical therapists. Pain was rated on three eleven-point scales, one for mean pain level in the last 24 hours, one for lowest level of pain in the last 24 hours and one for worst pain in the last 24 hours.

Table 1 presents the measurement instruments used, as well as the timing of the measurements. 
Table 1: Effect measures, methods and moments

* Primary measures of effect.

\begin{tabular}{|c|c|c|c|c|}
\hline & Method & $\begin{array}{l}\text { T0 } \\
2 \text { weeks } \\
\text { prior to } \\
\text { surgery }\end{array}$ & $\begin{array}{l}\text { T1 } \\
4 \text { days after } \\
\text { surgery }\end{array}$ & $\begin{array}{l}\text { T2 } \\
17 \text { days } \\
\text { after surgery }\end{array}$ \\
\hline Mobility* & $\begin{array}{l}\text { Long arm } \\
\text { Goniometer }\end{array}$ & $\mathrm{X}$ & $\mathrm{X}$ & $\mathrm{X}$ \\
\hline Function* & HSS score & $\mathrm{X}$ & $\mathrm{X}$ & $\mathrm{X}$ \\
\hline Length of hospital stay & Hospital record & & $\mathrm{X}$ & \\
\hline Pain & 11 point scale & $\mathrm{x}$ & $\mathrm{X}$ & $\mathrm{X}$ \\
\hline Pain medication & Hospital record & & $\mathrm{X}$ & $\mathrm{X}$ \\
\hline $\begin{array}{l}\text { Satisfaction treatment } \\
\text { physical therapist }\end{array}$ & 11 point scale & & $\mathrm{X}$ & \\
\hline $\begin{array}{l}\text { Satisfaction attention } \\
\text { physical therapist }\end{array}$ & 11 point scale & & $\mathrm{X}$ & \\
\hline Muscle strength & $\begin{array}{l}\text { Hand-held dyna- } \\
\text { mometer }\end{array}$ & $\mathrm{x}$ & $\mathrm{X}$ & $\mathrm{X}$ \\
\hline $\begin{array}{l}\text { Hours in CPM per } 24 \\
\text { hours }\end{array}$ & Hospital record & & $\mathrm{X}$ & \\
\hline
\end{tabular}

\section{Data analysis}

The data of all patients were entered in and analysed with SPSS statistical software package, version 9.0 (Chicago, IL, USA) ${ }^{21}$ by a blinded analyst. Data were analyzed according to the "intention to treat" principle ${ }^{22}$. Primary effect measures were calculated as the differences between T0, T1 and T2. Between-group differences were used in all analysis. A two tailed T-test was used for continuous data. Mann-Whitney U tests for HSS scores.

\section{Results}

Forty patients were included in the study, 20 of whom received physiotherapy twice a day during all four days of their hospital stay (PT group), while 20 received CPM in addition to the physiotherapy (CPM group). 
Figure 2: study overview from registration to follow up at day 17.

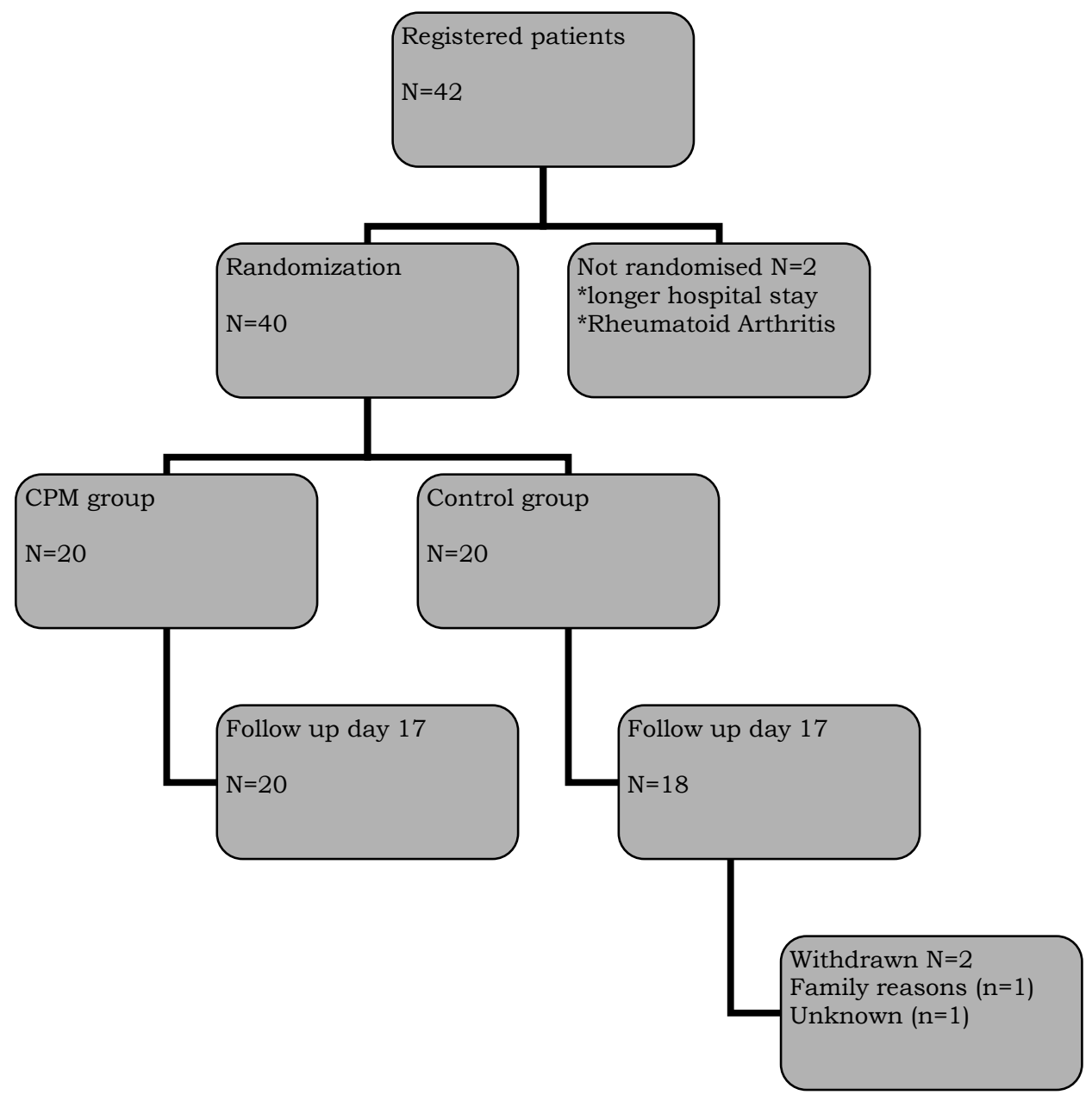

Baseline characteristics of the patients are shown in Table 2. Randomization resulted in a good balance between baseline characteristics in the two groups.

Baseline data on strength were lacking for one patient, due to problems with the dynamometer. Data on muscle strength at discharge were missing for the same patient. 
Table 2: Baseline characteristics

* In the last 24 hours

\begin{tabular}{lcrlr} 
& CPM group & & $\begin{array}{l}\text { PT group } \\
\mathrm{n}=19\end{array}$ & \\
& $\mathrm{n}=21$ & & \\
& Mean & $(\mathrm{SD})$ & $\mathrm{Mean}$ & $(\mathrm{SD})$ \\
Gender male/female & $6 / 15$ & & $7 / 12$ & \\
Age & 65,0 & $(9,2)$ & 65,7 & $(9,5)$ \\
Passive extension in degrees & 5,1 & $(5,3)$ & $5,6^{\circ}$ & $(6,3)$ \\
Passive flexion in degrees & 117,4 & $(15,6)$ & $120^{\circ}$ & $(15)$ \\
H.S.S. score (0-100pts) & 62,6 & $(12,7)$ & 66,1 & $(8,5)$ \\
Strength in Newton & 163,9 & $(52,6)$ & 165,6 & $(70,5)$ \\
Average pain (0-10 pts) & 3,6 & $(2,8)$ & 3,8 & $(2,7)$ \\
Minimal pain (0-10 pts) & 2,5 & $(2,6)$ & 1,7 & $(2,2)$ \\
Maximal pain (0-10 pts) & 7,6 & $(2,3)$ & 7,9 & $(1,6)$ \\
\hline
\end{tabular}

One patient was not evaluated on day 4 because he had suffered a heart attack on day three after the operation and was being treated at the intensive care ward on day four. Evaluation of this person was completed on day 17.

Two patients did not return for the follow-up visit, one because of the death of her husband during the postoperative period, the second one without notice.

\section{RoM}

Table 3 shows a comparison of the data on Range of Motion. On day 4 as well as day 17 after the operation, the CPM group performed better on passive RoM. Passive flexion declined immediately after surgery, being approximately $40^{\circ}$ less on day 4 than before surgery. The difference was $36^{\circ}$ in the CPM group and over $45^{\circ}$ in the control group. In absolute values, flexion was $6^{\circ}$ less and extension $1.6^{\circ}$ less in the control group.

On day 17, RoM had improved in both groups, but the difference between the groups remained. The overall RoM difference on day 17 was more than $10^{\circ}$.

Only the difference in extension on day 17 was statistically significant $(p=0.029)$.

Table 3: passive ROM on days 4 and 17 (* statistically significant) $\triangle$ Difference with preoperative value

\begin{tabular}{|c|c|c|c|c|c|c|c|c|}
\hline & \multicolumn{4}{|c|}{ Day 4 after surgery } & \multicolumn{4}{|c|}{ Day 17 after surgery } \\
\hline & degrees & & & & deg1 & $s \quad(s d)$ & & \\
\hline & $\begin{array}{l}\text { CPM } \\
(n=20)\end{array}$ & $\begin{array}{l}\text { contrc } \\
(n=19\end{array}$ & & $\begin{array}{l}P \\
\text { value }\end{array}$ & $\begin{array}{l}\text { CPM } \\
(n=20)\end{array}$ & & $\begin{array}{l}\text { control } \\
(\mathrm{n}=18)\end{array}$ & $\begin{array}{l}P \\
\text { valu }\end{array}$ \\
\hline Exter & $4,7^{\circ} \quad(4,5)$ & $6,2^{\circ}$ & $(4,2)$ & 0.27 & $4,2^{\circ}$ & $\left(3,4^{*}\right)$ & $7,9^{\circ} \quad(5,9$ & 0.03 \\
\hline Flexio & $80,3^{\circ}$ & $74,5^{\circ}$ & $(13,3)$ & 0.16 & $90,2^{\circ}$ & $(13,2)$ & $83,7^{\circ}(15,1)$ & 0.16 \\
\hline$\Delta$ extension & $(8,0)$ & $-0,6$ & $(7,2)$ & 0.66 & 1,2 & $(5,7)$ & $-2,1 \quad(5,8)$ & 0.09 \\
\hline$\Delta \Delta$ flexion & $-36,2(17,7)$ & $-45,5$ & $(12,4)$ & 0.07 & $-27,6$ & $(15,6)$ & $-36,1 \quad(10,6)$ & 0.06 \\
\hline
\end{tabular}

Function, as measured with the Hospital for Special surgery Score (HSS score)

The control group received in-hospital PT twice a day for 20 minutes for 4 days. The CPM group additionally received 4 hours of CPM daily. 
HSS score declined after surgery, as is illustrated in Table 4. The decline in the control group was greater than that in the CPM group. After 17 days, the score was comparable to preoperative values in the CPM group whilst the score in the control group was still much lower. All between-group differences were statistically significant.

Table 4: HSS score on day 4 and 17

$\triangle$ HSS shows difference with pre-operative values

\begin{tabular}{|c|c|c|c|c|c|c|c|}
\hline & 4 days & & & 17 days & & & \\
\hline & $\begin{array}{l}\text { CPM } \\
(n=20) \\
\text { mean }\end{array}$ & $\begin{array}{l}\text { control } \\
(n=19) \\
\text { mean sd }\end{array}$ & $\begin{array}{l}P \\
\text { value }\end{array}$ & $\begin{array}{l}\text { CPM } \\
(\mathrm{n}=20) \\
\text { mean }\end{array}$ & sd & $\begin{array}{l}\text { Control } \\
(\mathrm{n}=18) \\
\text { mean sd }\end{array}$ & $P$ value \\
\hline HSS & $56,5 \quad(9,8)$ & $44,5(14,9)$ & 0.001 & 66,2 & $(10,1)$ & $54,2 \quad(12,8)$ & 0.003 \\
\hline$\Delta \mathrm{HSS}$ & $-5,7 \quad(17,9)$ & $21,6(13,9)$ & 0.006 & 4,6 & $(15,4)$ & $-11,9 \quad(9,7)$ & 0.001 \\
\hline
\end{tabular}

\section{Secondary outcome measures}

After 4 days, muscle strength in the CPM group was twice as high as in the control group (48 vs. 24 N). After 17 days, strength was about $55 \%$ of the preoperative value and there were no significant differences between the two groups (Table 5).

Pain is presented as median pain level at rest and highest and lowest pain levels during the last 24 hours. Although it was not a primary outcome measure, differences in pain perception were regarded as a prominent outcome in this study. Pain was perceived as less of a problem in the CPM group at 4 as well as 17 days after surgery. Betweengroup differences were statistically significant at every evaluation point. The length of the hospital stay and the level of satisfaction with treatment and attention provided by the therapists were similar in both groups.

Compliance was measured as the number of hours in the CPM machine per day. Patients in the CPM group received an average of approximately 3.6 hours of CPM treatment per day. 


\begin{tabular}{|c|c|c|c|c|c|c|c|c|c|c|}
\hline & \multicolumn{5}{|c|}{4 days } & \multicolumn{5}{|c|}{17 days } \\
\hline & $\begin{array}{l}\text { CPM } \\
\mathrm{n}=20 \\
\text { mean }\end{array}$ & (sd) & $\begin{array}{l}\text { Contr } \\
\mathrm{n}=19 \\
\text { mean }\end{array}$ & (sd) & $\begin{array}{l}P \\
\text { value }\end{array}$ & $\begin{array}{l}\mathrm{CPM} \\
\mathrm{n}=20 \\
\text { mean }\end{array}$ & sd & $\begin{array}{l}\text { Contro } \\
n=18 \\
\text { mean }\end{array}$ & sd & $\begin{array}{l}P \\
\text { value }\end{array}$ \\
\hline Strength & 48,2 & $(30,1)$ & 24,2 & $(21,3)$ & 0.008 & 99,0 & $(45,6)$ & 80,3 & $(31,0)$ & 0.161 \\
\hline Pain average & 2,6 & $(1,8)$ & 4,7 & $(2,6)$ & 0.005 & 2,3 & $(2,6)$ & 4,5 & $(2,4)$ & 0.009 \\
\hline Pain maximum & 5,5 & $(2,7)$ & 8,2 & $(1,1)$ & 0.001 & 4,3 & $(3,3)$ & 7,2 & $(2,6)$ & 0.005 \\
\hline Pain minimum & 1,1 & $(1,3)$ & 2,3 & $(2,2)$ & 0.042 & 0,9 & $(1,6)$ & 2,9 & $(2,6)$ & 0.007 \\
\hline Length of stay & 6,0 & $(3,6)$ & 5,6 & $(1,1)$ & 0.709 & & & & & \\
\hline $\begin{array}{l}\text { Satisfaction } \\
\text { with treatment }\end{array}$ & 9,0 & $1,0)$ & 8,6 & $(1,0)$ & 0.268 & & & & & \\
\hline $\begin{array}{l}\text { Satisfaction } \\
\text { with attention }\end{array}$ & 9,0 & $1,1)$ & 8,6 & $(1,0)$ & 0.282 & & & & & \\
\hline
\end{tabular}

\section{Discussion}

Both groups did better than patients reported on by several other studies 810 16. However, differences in ROM between the CPM and control group were not significant in this trial. This difference in RoM was approximately $8^{\circ}$ at $\mathrm{T} 1$ and $10^{\circ}$ at $\mathrm{T} 2$. This may be due to the intensive treatment in the Control group. Johnson et al. 10 immobilised the patients in their Control group for 7 days, while Pope et al. 8 immobilized all patients in an extension splint between physiotherapy sessions during the first three days after surgery. Although CPM was not continued in the first week after discharge, the differences in RoM in the current study were found to persist through day 17 . The data do not show whether, and if so when, the Control group made up this difference.

Differences in function score between the CPM and Control groups were found to be marked. Secondary analysis showed that over 50\% of this difference was attributable to the difference in pain perception, which is part of the HSS questionnaire. One might, therefore, question whether such difference really represents a change in overall function, or merely the use of a different kind of pain measurement. Better tools are required to measure functions of daily living in this treatment group.

\section{Secondary measures of effect}

Quadriceps' strength levels at four days after surgery were much higher in this study than reported by McInnes et al. ${ }^{11}$. This apparent difference could be due to the fact that we measured strength at about $60^{\circ}$ of flexion, whereas they measured at $45^{\circ}$ of flexion. 
However, McInnes and colleagues did not find any significant differences in strength, whereas we found a difference of $50 \%$ between the $\mathrm{CPM}$ and control groups.

A possible explanation for the difference in strength between both groups could be the fact that CPM is not as 'passive' a treatment as we think it is, and may involve muscular activity while "resting" in the machine. Why we found a large difference whereas McInnes et al. ${ }^{11}$ reported no difference remains unclear. The effect on pain was also markedly greater than that reported by previous studies. McInnes et al. ${ }^{11}$ reported a moderate difference in favour of the CPM group; however, the overall pain scores in their study were smaller compared to our data.

The effect on pain cannot be explained by differences in medication, because pain management was standardized in our trial. A possible explanation is that prolonged passive motion leads to pain reduction through arthokinematic reflex activity ${ }^{23}$.

Compliance in the CPM group was less than the planned 4 hours of CPM treatment a day.

One possible reason for this could be the use of the hospitals "living room" facilities during the daytime, which was located about $50 \mathrm{~m}$ from the bedrooms. Since it is more difficult to install the CPM machine in a chair than in a hospital bed, physical therapists and nursing staff occasionally skipped this procedure.

The physiotherapy treatment in this trial was intensive. In-hospital regimens in the Netherlands usually prescribe one 20-minute physiotherapy session a day. Hence, results must be evaluated in the context of a Control treatment which had 40 minutes of physiotherapy in its protocol.

Only short-term effects of CPM are described here. We were interested in the short-term effect because a systematic review had found no consistent long-term effects of the treatment, but strong evidence of shortterm effect, mainly on RoM. Considering the outcome of the present study, we would also be interested in medium term outcome, to examine whether the control group made up this difference with the CPM group and if so over whet time interval.

Furthermore, we intend to perform a new trial in which CPM application is prolonged for two weeks, as 'dosage' may be a possible factor of influence.

\section{Conclusions}

This study indicates that CPM delivered in the first 4 days following surgery seems to lead to better short-term mobility. The increased mobility is present through the 17 th day after surgery.

Results on function should be interpreted with caution, because pain and function seem closely related in the scoring system used here. 
Nevertheless, there was a considerable difference in favour of the CPM group in terms of this functional outcome measure.

The most striking outcome was the difference in postoperative pain perception, with CPM use leading to an immediate decline in postoperative pain.

Finally, muscular strength in our CPM group is better than that in the control group, at day 4 as well as day 17 .

Future research should concentrate on longer term outcome, longer use of CPM and finding a better outcome tool for functional assessment. 


\section{References}

1. Salter RB. The Biologic Concept of Continuous Passive Motion of Synovial Joints. Clinical Orthopedics and Related Research 1989;242(may 1989): 12-26.

2. O'Driscoll SW, Giori NJ. Continuous Passive Motion (CPM): Theory and principles of clinical application. Journal of Rehabilitation Research and Development 2000;37(2):179-188.

3. Vince K, Kelly MA, Beck J, Insall JN. Continuous Passive Motion after Total Knee Arthroplasty. Journal of Arthroplasty 1987;2(4):281-284.

4. Ritter MA, Gandolf VS, Holston KS. Continuous Passive Motion versus Physical Therapy in Total Knee Arthroplasty. Clinical Orthopedics and Related Research 1989(244):239-243.

5. Nielsen PT, Rechnagel K, Nielsen S. No effects ofcontinuous passive motion after arthroplasty of the knee. Acta Orthopedica Scandinavia 1988;59(5):580-581.

6. Lenssen AF, Köke, A.J.A., de Bie, R.A. Effectiviteit van continuous passive motion in de klinische nabehandeling van patienten met een totale knie prothese: een systematische review. Ned. Tijdschr. Fysiotherapie 2001;6(dec 2001):152-158.

7. May LA, Busse W, Zayac D, Whitridge MR. Comparison of Continuous Passive Motion Machines and Lower Limb Mobility Boards in the Rehabilitation of Patients with Total Knee Arthroplasty. Can $J$ of Rrehabilitation 1999;12(4):257-263.

8. Pope RO, Corcoran S, Mc Caul K, Howie DW. Continuous Passive Motion after Primary Total Knee Arthroplasty

Does it offer any benefits. Journal of Bone and Joint Surgery 1997;79$\mathrm{B}(6): 914-917$.

9. Worland RL, Arredondo J, Angles F, Jimenez FL, Jessup DE. Home Continuous Passive Motion Machine versus Professional Physical Therapy Following Total Knee Replacement. Journal of Arthroplasty 1998;13(7):784788.

10. Johnson DP, Eastwood DM. Beneficial Effects of Continuous Passive Motion after Total Condylar Knee Arthroplasty. Annals of the Royal College of Surgeons of England 1992;74:412-416.

11. Mc Innes J, Larson MG, Daltroy LH, Brown T, Fossel AH, Eaton HM, et al. A Controlled Evaluation of Patients Undergoing Total Knee Arthroplasty. JAMA 1992;268(11, SEPTEMBER 16):1423-1428.

12. Montgomery F, Eliasson M. Continuous Passive Motion Compared to Active Physical Therapy after Knee Arthroplasty. Acta Orthopedica Scandinavia 1996;67(1):7-9.

13. Chen B, Zimmerman JR, Soulen L, DeLisa JA. Continuous Passive Motion after Total Knee Arthroplasty. American Journal of Physical Medicine and Rehabilitation 2000;79(5):421-426.

14. Chiarello CM, Gunderson L, O'Halloran T. The Effect of Continuous Passive Motion Duration and Increment on Range of Motion in Total Knee Arthroplasty Patients. JOSPT 1997;25(2):119-127.

15. Colwell CW, Morris BA. The Influence of Continuous Passive Motion on the Results of Total Knee Arthroplasty. Clinical Orthopedics and Related Research 1992(276):225-228. 
16. Harms M, Engstrom B. Continuous Passive Motion as an Adjunct to Treatment in the Physical Therapy Management of the Total Knee Arthroplasty Patient. Physiotherapy 1991;77(4, APRIL 1991):301-307.

17. Kumar PJ, Mc Pherson EJ, Dorr LD, Wan Z, Baldwin K. Rehabilitation After Total Knee Arthroplasty

A Comparison of 2 Rehabilitation Techniques. Clinical Orthopedics and Related Research 1996(331):93-101.

18. Beaupre LA, Davies DM, Jones CA, Cinats JG. Exercise combined with continuous passive motion or slider board therapy compared with exercise only: a randomized controlled trial of patients following total knee arthroplasty. Phys Ther 2001;81(4):1029-37.

19. Walker RH, Morris BA, Angulo DL, Schneider J, Colwell CW, Jr. Postoperative use of continuous passive motion, transcutaneous electrical nerve stimulation, and continuous cooling pad following total knee arthroplasty. J Arthroplasty 1991;6(2):151-6.

20. Ranawat CS, Insall JN, Shine J. Duo Condylar Knee Arthroplasty: Hospital for Special Surgery Design. Clinical Orthopedics and Related Research 1976;120:76-82.

21. Norusis MJ. SPSS 9.0 guide tot data analyses. Chicago: SPSS Inc.1999.

22. Bouter LM, Dongen van MCJM. Epidemiologisch Onderzoek, opzet en interpretatie. 2 ed. Houten/ Antwerpen: Bohn Stafleu Van Loghum, 1991.

23. Rondhuis GB. Knierevalidatie. 1 ed. Utrecht: de Tijdstroom, 1996. 
Reproducibility of goniometric measurement of the knee in the in-hospital phase following total knee arthroplasty.

\author{
AF Lenssen \\ EM van Dam \\ YHF Crijns \\ MJA Verhey \\ RJT Geesink \\ $P A$ van den Brandt \\ RA de Bie
}




\section{Abstract}

Objective: To assess interobserver reproducibility (agreement and reliability) of measurements of knee range of motion (RoM).

Design: Test-retest analysis

Setting: University hospital departments of orthopaedics and physiotherapy

Participants: Two experienced physical therapists assessed 30 patients, three or four days after total knee arthroplasty

Main outcome measure: RoM measurement using a long-arm $(50 \mathrm{~cm})$ goniometer

Agreement was calculated as the mean difference between observers \pm $95 \%$ CI of this mean difference. The intraclass correlation coefficient (ICC) was calculated as a measure of reliability, based on two-way random effects analysis of variance.

Results: The lowest level of agreement was that for measurement of passive flexion with the patient in supine position (mean difference $1.4^{\circ}$; limits of agreement $16.2^{\circ}$ to $19^{\circ}$ for the difference between the two observers. The highest levels of agreement were found for measurement of passive flexion with the patient in sitting position and for measurement of passive extension (mean difference $2.7^{\circ}$; limits of agreement $-6.7^{\circ}$ to $12.1^{\circ}$ and mean difference $2.2^{\circ}$; limits of agreement $-6.2^{\circ}$ to $10.6^{\circ}$, respectively).The ability to differentiate between subjects was fair to good for all movements (ICC ranging from 0.62 for measurement of passive extension to 0.89 for measurements of active flexion)

Conclusions: Interobserver agreement for flexion as well as extension was only fair.

When two different observers assess the same patients in the acute phase after total knee arthroplasty, differences in RoM of less than eight degrees cannot be distinguished from measurement error. Reliability was found to be fair for extension RoM and good for flexion RoM measurements of the knee. 


\section{Background}

Total knee arthroplasty (TKA) is a common orthopaedic procedure. In the Netherlands, over 7500 TKAs are performed annually. After the operation, many patients require physiotherapy (PT) to regain functional independence and resume work-related physical activities ${ }^{1}$. One of the main components of the PT programme is mobilisation of the knee joint to increase the range of motion (RoM).

Physical therapists and orthopaedic surgeons use measurement of RoM not only to quantify limitations at the start of treatment but also as an outcome measure to justify their actions or quantify the effectiveness of interventions.

The use of validated and reproducible measurement instruments is an important prerequisite for the evaluation of clinical practice, as well as for the interpretation of study results ${ }^{2}$. The long-arm universal goniometer (UG) is an instrument frequently used to quantify restrictions in RoM ${ }^{3} 4$. Over the years, many studies ${ }^{2-11}$ have addressed the reproducibility of RoM measurements of the knee. Studies 25 have shown that goniometric measurements are more reliable than visual estimates. Several authors reported intraobserver reproducibility to be better than interobserver reproducibility 58 . Generally speaking, reproducibility is better for knee flexion than knee extension 4910 .

Most studies have reported on the reliability of RoM measurements, whereas only one study also reported on interobserver agreement 10 . Agreement between observers is an essential component of reproducibility of measurement when utilized in a clinical setting. A method to describe clinically acceptable agreement between observers was introduced by Bland and Altman ${ }^{12}$. The method is based on simple calculations of the standard deviation of the difference between two observers and plotting these differences against their mean ${ }^{13}$. The magnitude of the SD expresses the extent to which the observers are able to achieve the same value ${ }^{14}$. Subsequently, the $95 \%$ limits of agreement are calculated, defined as the mean difference between observers \pm 1.96 SDs of this mean difference. Only differences that exceed the limits of agreement can be interpreted as "real" differences exceeding measurement error. The smallest difference found is called the smallest detectable difference. Deciding the magnitude of the limits of agreement, representing acceptable agreement, is a clinical rather than a statistical decision.

It is important in reproducibility research to take into account what is to be measured, by whom and in what situation, because results of any kind of research will primarily be valid for this particular situation. Too often, the focus is on the measurement tool itself, whereas observers, patient categories and test situations are neglected ${ }^{15}$.

Most of the existing reports on the reproducibility of RoM measurements of the knee relate to studies on native knees. Only Edwards et al. ${ }^{16}$ reported on the reproducibility of measurements in patients after TKA implantation. 
Most studies 416 have measured in a laboratory-type environment or test protocol ${ }^{9}$. In clinical practice, patients are seen by different therapists and orthopaedic surgeons during their hospital stay. All of them measure range of motion to evaluate progress in the first days after surgery. However, the reproducibility of RoM measurements may vary with the clinical problem, the examiner and the environment in which reproducibility is measured.

In the present study, active and passive RoM was measured to reflect clinical practice. Gaidosik et al. ${ }^{3}$ state that passive movements are more difficult to reproduce because of the stretching of soft tissues. The limits of range of motion depend on the force applied to the limb. The objective of the present study was to assess interobserver reproducibility (in terms of reliability and agreement) of active and passive measurements of knee RoM, performed by trained physical therapists in a clinical setting in total knee arthroplasty patients, within the first four days after surgery.

\section{Methods}

\section{Patients}

Between January 1 and March 30, 2004, consecutive eligible hospitalised patients in the acute phase after total knee arthroplasty at the orthopaedic ward of the Maastricht university hospital were invited to participate in the study by the first author (AFL). Patients were eligible if they met the following inclusion criteria: status after TKA because of osteoarthritis, ability to co-operate (sufficient Dutch language skills, no dementia) and having given informed consent. Patients with a history of neuromuscular pathologies and patients with revision TKA were excluded.

The study was part of a larger trial approved by the Maastricht University and university hospital medical ethics committee.

\section{RoM Measurement}

Two experienced observers ( $\mathrm{YC} \& \mathrm{MV}$ ), both physical therapists, independently measured active and passive flexion and extension RoM of the operated knee using a long-arm goniometer. Measurement procedures were standardised prior to the study. Both therapists had trained the procedures on 30 healthy subjects before the start of the trial.

All measurements were taken on the third or fourth day after surgery. Observers used a predefined procedure for the measurements (Table 1). To prevent the occurrence of systematic differences between observers because of repeated testing, the observers were allocated in random order. The time interval between the measurements by the first and second observer was less than five minutes, and the patients did not receive any therapy between the two measurements. 
Only one observer was present in the examination room at any time, together with a research assistant. The research assistant recorded the number of degrees reported by the observer.

Observers and patients were kept unaware of the measurement outcome generated by the previous observer.

RoM was measured using a long-arm goniometer (arm length $50 \mathrm{~cm}$ ). Active RoM was determined prior to passive RoM. Patients recorded severity of pain while being tested by giving a report mark ranging from 0 'no pain' to 10 'severe pain'.

Extension measurements were taken with the patient in supine position. Flexion was measured in supine position and while the patient was seated upright on the examination table with hips in $90^{\circ}$ of flexion. Whereas most studies 4616 have measured flexion in supine position, at our hospital we tend to measure flexion while the patient is seated. We therefore measured flexion RoM in both positions.

All goniometric measurements were performed according to the technique described by Norkin and White ${ }^{17}$, with the centre of the fulcrum positioned over the lateral condyle of the femur. The proximal fixed arm of the goniometer was aligned with the axis of the femur by using the greater trochanter as a reference point. The distal mobile arm was aligned using the lateral malleolus.

\section{Table 1: Measurement procedure}

\begin{tabular}{lll}
\hline Knee & Patient position & Bony orientation \\
Flexion & Seated, hips in $90^{\circ}$ of flexion & Proximal trochanter major \\
Extension & Supine & Lateral femur epicondyle \\
& Supine & Caput fibulae \\
& Lateral malleolus \\
\hline
\end{tabular}

\section{Statistical analysis}

For each observer, the mean and standard deviations were calculated for each RoM assessed. To quantify reproducibility, we distinguished two different types of reproducibility measures with different interpretations: measures of agreement and measures of reliability. Measures of agreement refer to the absolute measurement error (presented in the units of measurement of the instrument) that is associated with one measurement taken from an individual patient 18 19. Measures of agreement provide insight into the ability of two or more observers to achieve the same value. Measures of reliability refer to the relative measurement error, i.e. the variation between patients in relation to the total variance of the measurements. They provide insight into the ability of two or more observers to differentiate between subjects in a group.

\section{Agreement}

The mean difference between the two observers and the SD of this difference were calculated. The magnitude of the SD expresses the extent to which the observers were able to achieve the same value ${ }^{14}$. 
Subsequently, the 95\% limits of agreement were calculated, defined as the mean difference between observers $+1.96 \mathrm{SDs}$ of this mean difference ${ }^{12}$.

\section{Reliability}

The intraclass correlation coefficient (ICC) is defined as the ratio of the variance between patients to the total variance. ICC values can theoretically range from 0 to 1 , with a higher value indicating that less variance is due to other factors such as differences between observers. An intraclass correlation coefficient of at least 0.70 is considered to be satisfactory for group comparisons, and a value of 0.90-0.95 for individual comparisons ${ }^{20}$. The ICC was calculated with two-way random effects ANOVA.

We used SPSS 12.0 statistical software for Windows to calculate the ICCs (SPSS inc., Chicago IL)

\section{Results}

Over periods of three months (January to April 2004) 30 TKA patients were recruited. The mean age of included patients was 69 years (range 51-77) and $80 \%$ were female. TKA of the left knee had been performed in 17 cases, while 13 patients had a right knee TKA. All measurements were taken three or four days after surgery.

The results of the interobserver agreement and reliability with regard to the different RoM measurements are presented in table 2 and figures 14.Remarkably, both observers measured higher flexion angles with patients in sitting position. 
Table 2 Inter-rater reproducibility of knee RoM measurement

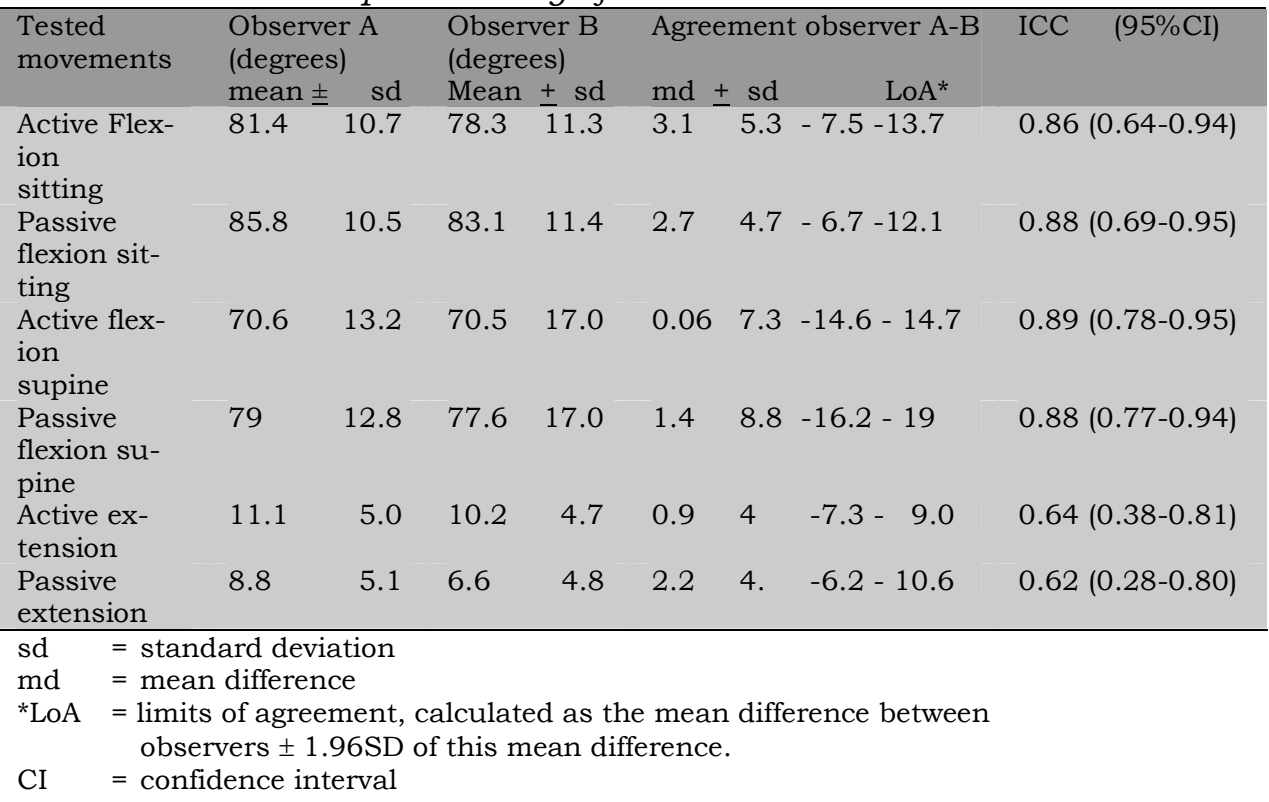

\section{Agreement}

The observers produced quite similar measurements for extension and flexion in the supine position; however, observer A measured a consistently wider range of motion than observer B.

The highest level of agreement was found for active and passive extension and passive flexion whilst sitting (figure 1). Limits of agreement \pm $1.96 \mathrm{SD}$ were $3.2^{\circ} \pm 9.4^{\circ}$ for passive flexion and $0.9^{\circ} \pm 8.2^{\circ}$ for active extension.

Lowest levels of agreement were found for active and passive flexion whilst supine. Limits of agreement $\pm 1.96 \mathrm{SD}$ were $0.06^{\circ} \pm 14.6^{\circ}$ for active flexion and $2.2^{\circ} \pm 17.6^{\circ}$ for passive flexion.

Figure 1 shows the difference between observers, plotted against the mean value for both observers for passive flexion measured in a sitting position and for passive extension measured in supine position. Figures 1 and 3 both show that errors of the measurement were independent of the magnitude of the range of motion (homoscedasticity). 
Figure 1: Differences between two observers, plotted against the mean values for both observers for each patient for flexion RoM in sitting position. The figure shows the mean difference between observers (solid line at centre) and the limits of agreement (dashed outer lines corresponding to \pm 1 . $96 \mathrm{SDs}$ of the mean difference between the first and second observers).

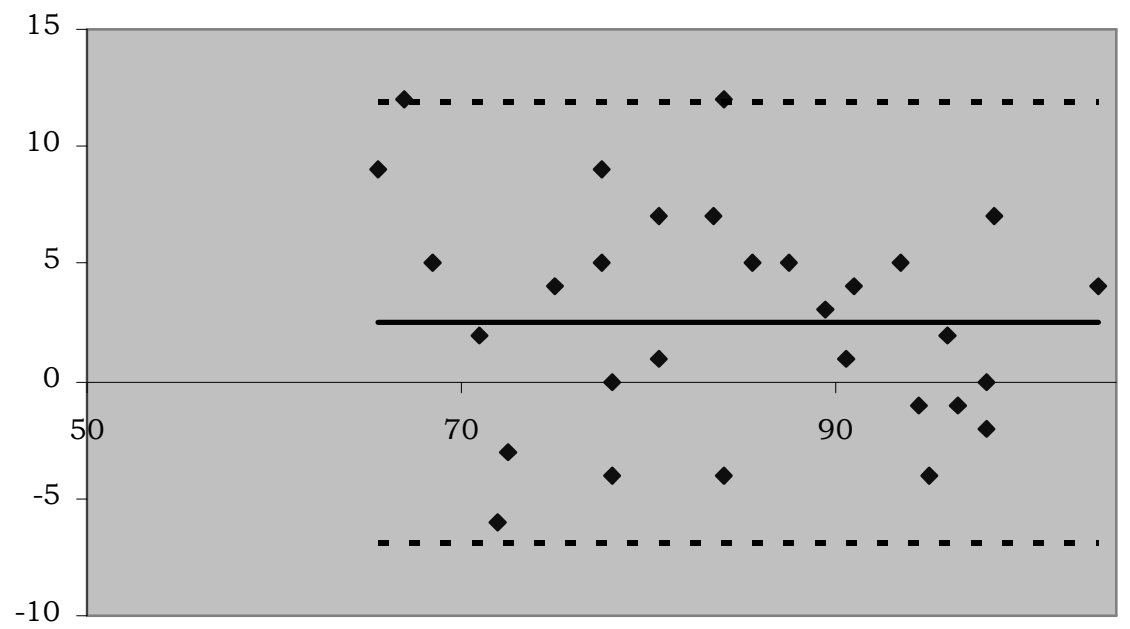

Figure 2: Scatter plot of interobserver reliability of measurement of passive flexion whilst sitting, as indicated by the ICCs

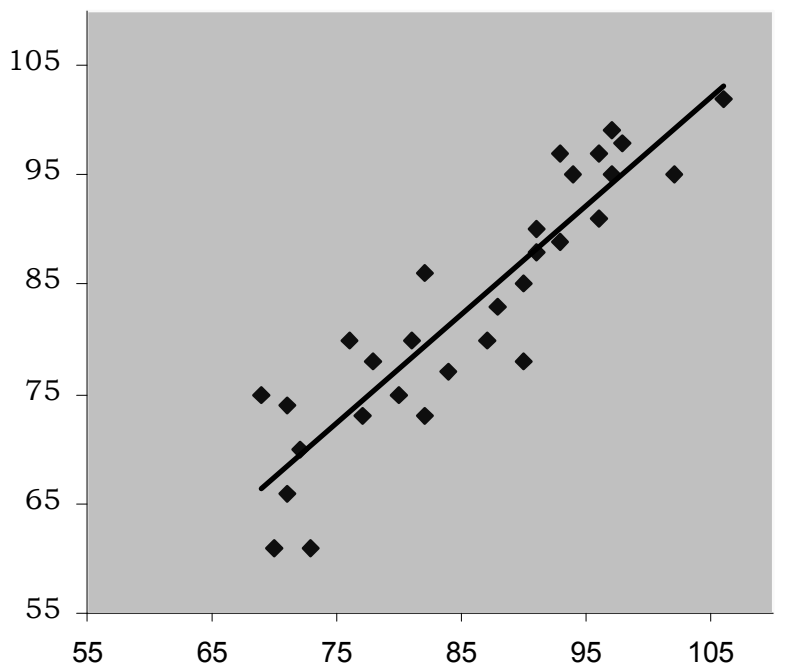




\section{Reliability}

ICC values were highest for active and passive flexion whilst sitting (figure 2), and lowest for active and passive extension. The ICC values ranged from 0.62 for passive extension to 0.89 for active flexion. The ICC for extension was lower than the ICCs for flexion, irrespective of active or passive measurement.

Pain during RoM measurement, as assessed by a 11 point scale, was worse in flexion and in passive measurements. No differences between the two observers were found either for perceived pain or for perceived pain and RoM reached during measurement (data not presented).

Figure 3: Differences between observers, plotted against the mean values of both observers for each patient for passive extension. The figure shows the mean difference between observers (solid line at centre) and the limits of agreement (dashed outer lines corresponding to \pm 1 . 96 SD of the mean difference between the first and second observers).

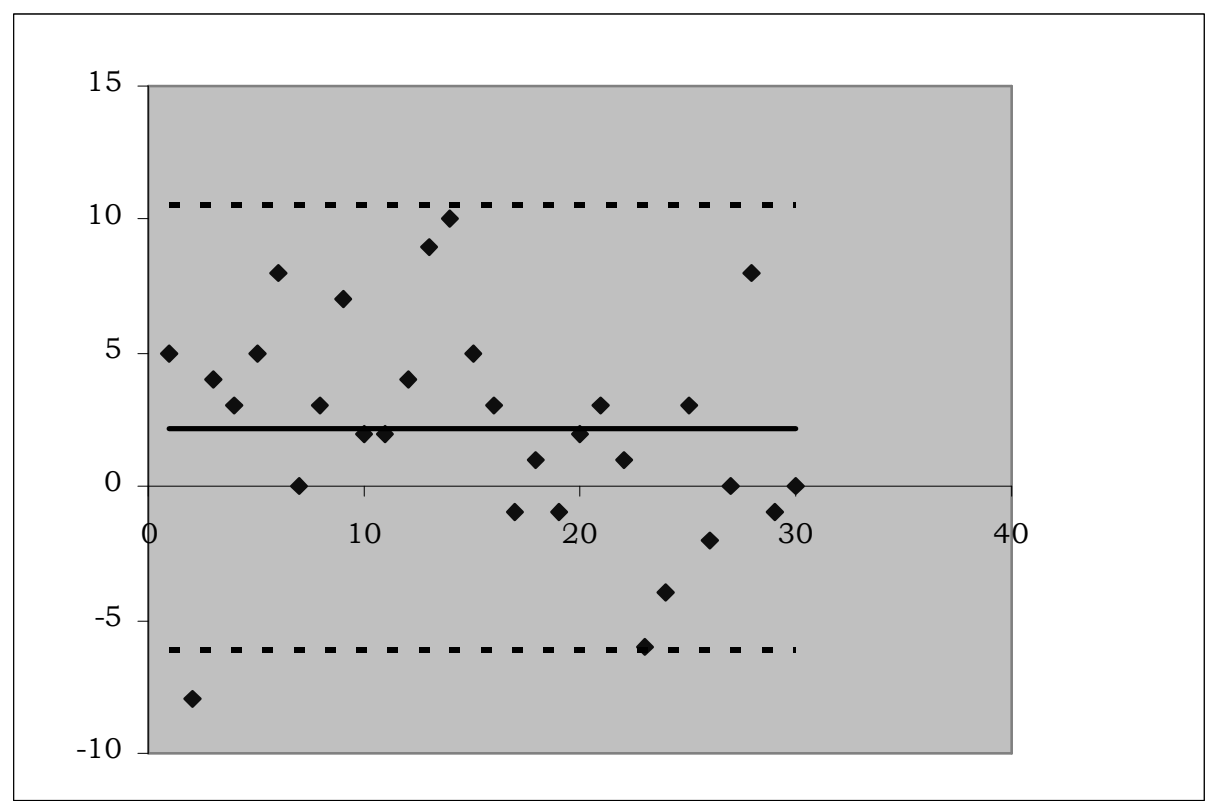


Figure 4: Scatter plot of interobserver reliability of measurement of passive extension

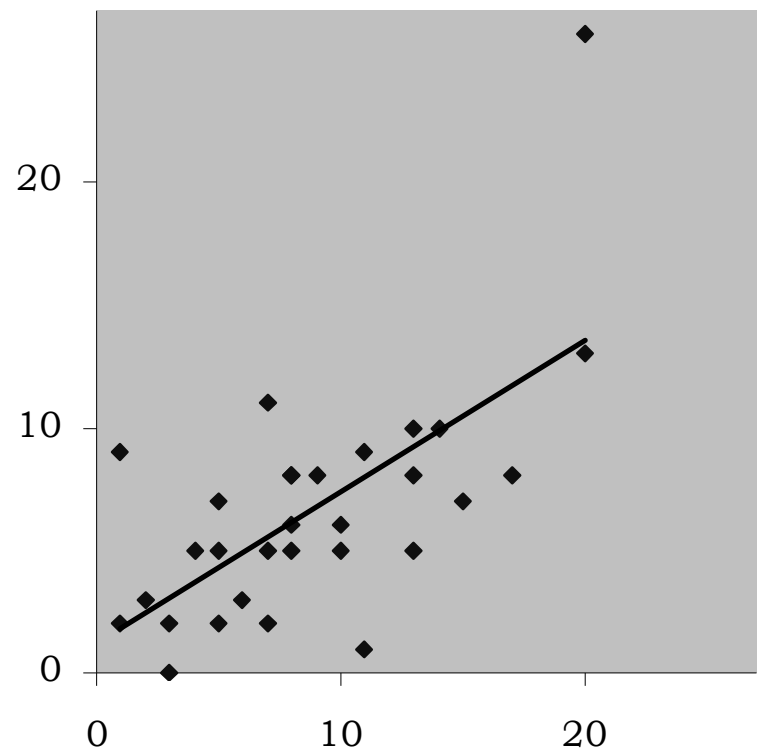

\section{Smallest detectable difference}

Based on the results of the interobserver agreement, the smallest detectable differences would lie between $8.2^{\circ}$ for active extension and $17.6^{\circ}$ for passive flexion while supine. This means that only changes in knee range of motion larger than these values can be detected beyond measurement error when different clinicians perform RoM measurements in a comparable clinical environment.

\section{Discussion}

This study investigated the interobserver reproducibility of the assessment of active and passive RoM of the knee in patients after TKA. The results show that there was considerable variation in the agreement between observers in all movements tested. The lowest limit of agreement was found for active extension. As regards flexion, the lowest limit was found for the measurement of passive flexion whilst sitting with $90^{\circ}$ flexion of the hip.

The reliability of the flexion measurement was good, while that for extension was only fair. This may have been caused by the wider RoM when measuring flexion. The ICC depends on the range of the true quantity in a sample, so if this range is wide, the correlation will be greater than if it is narrow 21. 
A similar explanation can be given for the narrower limits of agreement found for extension. The range of outcomes for extension is generally narrow, leading to narrower limits of agreement.

The results show that both observers measured less flexion RoM with patients in supine position than in sitting position. A possible explanation for the difference in measurement positions may be that the supine position is less stable and allows more degrees of freedom of movement in both hip and knee compared to the sitting position, in which the upper leg is fixed on the examination table, as already indicated by Gaidosik et al. ${ }^{3}$.

Although the measurement procedure was standardised, differences in effort by the patient are a potential source of bias in these measurements, and the influence of the examiner is added as a possible source of variation when measuring passive RoM. The amount of force used by the different observers to reach full RoM may well influence the reproducibility of the measurements ${ }^{3}$.

Unlike those of others ${ }^{4} 910$ our results do not show any differences between active and passive measurements. This might be caused by the level of experience, and the training of the participating therapists prior to the study, as well as the standardisation of measurements used by all therapists working with TKA patients in our hospital. Another explanation may be that in the acute phase after surgery, patients tend to guide passive measurement, for fear of extreme pain when going into extreme flexion or extension. This may well cause measurements of passive and active RoM to be more alike.

Although we concentrated on measuring in a clinical situation, our results with respect to reliability are similar to results reported in the literature, which were obtained in a laboratory environment 45911 16. This may have been partly caused by the use of a predefined testing procedure by experienced therapists, who had trained the procedures on healthy subjects and patients before the start of the study. Van Genderen et al. ${ }^{15}$ also described positive effects of including these steps in the measurement design.

Hence, we believe that RoM measurement in a clinical situation is possible without loss of reliability. However, it may be questioned whether agreement is not of greater importance in clinical measurement situations.

Linden-Peters et al. ${ }^{10}$ were the first to report on agreement in the measurement of knee RoM.

They reported better results for extension measurements. We believe this is probably caused by differences in the selection of the study population. Our population had a wider range of extension, leading to a wider limit of agreement.

\section{Study limitations}

The decision to use only two testers in this study might be debatable. For a correct simulation of everyday practice, we would have preferred to include all staff members involved in the follow-up of TKA patients at 
our clinic. We chose to include only two testers, however, because we believe that the inclusion of agreement is of the utmost importance in analysis of measurement in a clinical situation. This inclusion of limits of agreement as an outcome was only possible when using two testers.

Our research focused on the difference between observers, and did not include intraobserver reproducibility. We believe that studying intraobserver reproducibility would involve more interference with everyday practice, as individual observers would need to perform multiple measurements on the same patient and would have to be blinded for the outcome of each of their measurements.

This would interfere with our intention to mimic clinical measurement procedures and not to create a laboratory environment for our measurements.

In spite of our use of a measurement procedure with standardised measurement and pre-study training, we still found differences in RoM measurement between the two observers. Observer A persistently measured greater flexion RoM. We believe that this was caused by persistent differences between the testers in the choice of the fulcrum of rotation. This problem might be overcome by using the parallelogram goniometer introduced by Brosseau et al. ${ }^{4} 8$.

Since this study was conducted in one physiotherapy department, by therapists who measure RoM in TKA patients daily, the results may not necessarily be generalizable to all physical therapists.

Relation between reproducibility and responsiveness

To be useful for outcome assessment in clinical practice or research, an instrument should have high responsiveness, which is strongly related to the level of agreement ${ }^{22}$ Limits of agreement should be smaller than the minimum clinically relevant difference one wants to detect. As regards clinical practice, the large limits of agreement for all measurements in our study indicate that we should be very careful in comparing and interpreting results obtained by different observers. As regards research, we suggest to try to use only one observer. Unfortunately, practical reasons make it very difficult to investigate the level of intraobserver reproducibility. Future studies should investigate whether further standardisation or the use of other measurement tools such as a parallelogram goniometer might lead to smaller limits of agreement.

\section{Conclusions}

Interobserver agreement was low in the assessment of active and passive RoM of the knee in patients in the acute phase after total knee arthroplasty, notwithstanding the fact that it was measured by experienced physical therapists. By contrast, the reliability of RoM measurement was fair to good in this population. 


\section{References}

1. Naylor J, Harmer A, Fransen M, Crosbie J, Innes L. Status of physiotherapy rehabilitation after total knee replacement in Australia. Physiother Res Int 2006;11(1):35-47.

2. Rothstein JM, Campbell SK, Echternach JL, Jette AM, Knecht HG, Rose SJ. Standards for tests and measurements in physical therapy practice. Physical therapy 1991;71:589-622.

3. Gajdosik RL, Bohannon RW. Clinical measurement of range of motion. Review of goniometry emphasizing reliability and validity. Phys Ther 1987;67(12):1867-72.

4. Brosseau L, Balmer S, Tousignant M, O'Sullivan JP, Goudreault C, Goudreault $\mathrm{M}$, et al. Intra- and intertester reliability and criterion validity of the parallelogram and universal goniometers for measuring maximum active knee flexion and extension of patients with knee restrictions. Arch Phys Med Rehabil 2001;82(3):396-402.

5. Watkins MA, Riddle DL, Lamb RL, Personius WJ. Reliability of goniometric measurements and visual estimates of knee range of motion obtained in a clinical setting. Physical Therapy 1991;71(2):1522.

6. Kafer W, Fraitzl CR, Kinkel S, Clessienne CB, Puhl W, Kessler S. [Outcome assessment in total knee arthroplasty: is the clinical measurement of range of motion a reliable measurable outcome variable?]. $Z$ Orthop Thre Grenzgeb 2005;143(1):25-9.

7. Rheault W, Miller M, Nothnagel P, Straessle J, Urban D. Intertester reliability and concurrent validity of fluid-based and universal goniometers for active knee flexion. Phys Ther 1988;68(11):1676-8.

8. Brosseau L, Tousignant M, Budd J, Chartier N, Duciaume L, Plamondon $\mathrm{S}$, et al. Intratester and intertester reliability and criterion validity of the parallelogram and universal goniometers for active knee flexion in healthy subjects. Physiother Res Int 1997;2(3):150-66.

9. Rothstein JM, Miller PJ, Roettger RF. Goniometric reliability in a clinical setting. Elbow and knee measurements. Phys Ther 1983;63(10):1611-5.

10. Linden-Peters van den CJ, Genderen FR, Aufdemkampe G. Meten in beweging: betrouwbaarheidsonderzoek van active range of motion (AROM) volgens een andere visie. Nederlands Tijdschrift voor Fysiotherapie 2003;113(5):106-111.

11. Gogia PP, Braatz JH, Rose SJ, Norton BJ. Reliability and validity of goniometric measurements at the knee. Phys Ther 1987;67(2):192-5.

12. Bland JM, Altman DG. Statistical methods for assessing agreement between two methods of clinical measurement. Lancet 1986;1(8476):307-10.

13. Tammemagi MC, Frank JW, Leblanc M, Artsob H, Streiner DL. Methodological issues in assessing reproducibility--a comparative study of various indices of reproducibility applied to repeat ELISA serologic tests for Lyme disease. J Clin Epidemiol 1995;48(9):1123-32.

14. Terwee CB, de Winter AF, Scholten RJ, Jans MP, Deville W, van Schaardenburg $\mathrm{D}$, et al. Interobserver reproducibility of the visual estimation of range of motion of the shoulder. Arch Phys Med Rehabil 2005;86(7):1356-61. 
15. Genderen FR, van Meeteren NLU, Verhoef J.

Betrouwbaarheidsonderzoek : van een impliciete receptuur op weg naar een meer expliciete visie ; een overzicht van betrouwbaarheidsstudies van de hand-held dynamometer als voorbeeld. Ned Tijdschr Fysiotherapie 2001;111(5):116-124.

16. Edwards JZ, Greene KA, Davis RS, Kovacik MW, Noe DA, Askew MJ. Measuring flexion in knee arthroplasty patients. $J$ Arthroplasty 2004;19(3):369-72.

17. Norkin CC, White DJ. Measurement of joint motion: a guide to goniometry. Philadelphia: FA Davis, 1995.

18. de Winter AF, Heemskerk MA, Terwee CB, Jans MP, Deville W, van Schaardenburg DJ, et al. Inter-observer reproducibility of measurements of range of motion in patients with shoulder pain using a digital inclinometer. BMC Musculoskelet Disord 2004;5:18.

19. de Vet HC. Observer reliability and agreement. In: Armitage P, Colton T, editors. Encyclopedia of biostatistics. Boston: John Wiley \& sons, 1998:3123-3128.

20. Trust SACotMO. Assessing health status and quality-of-life instruments: attributes and review criteria. Qual Life Res 2002;11(3):193-205.

21. Bland JM, Altman DG. Comparing two methods of clinical measurement: a personal history. Int $J$ Epidemiol 1995;24 Suppl 1:S714.

22. de Vet HC, Bouter LM, Bezemer PD, Beurskens AJ. Reproducibility and responsiveness of evaluative outcome measures. Theoretical considerations illustrated by an empirical example. Int $J$ Technol Assess Health Care 2001;17(4):479-87. 


\title{
5
}

Efficiency of immediate postoperative inpatient physiotherapy following total knee arthroplasty: an RCT

\author{
AF Lenssen \\ YHF Crijns \\ EMH Waltjé \\ MJA van Steyn \\ RJT Geesink \\ PA van den Brandt \\ RA de Bie
}

BMC Musculoskeletal Disorders 2006, 7:71 


\begin{abstract}

\section{Background}

The main goal of physiotherapy treatment (PT) in the clinical stage following total knee arthroplasty (TKA) is to prepare patients for discharge from the hospital as soon as possible after their operation. Although aggressive rehabilitation is believed to be important, evidence of effects of different exercise programmes following TKA is limited. This led to the question whether the intensity of PT (once versus twice daily) following TKA affects short-term recovery, measured as range of motion.
\end{abstract}

\title{
Methods
}

A randomised controlled trial compared an exercise regimen of two sessions per day with a similar programme administered once daily. Primary outcome measure was ROM.

\section{Results}

At the time of hospital discharge, there was no difference between the experimental and control groups in range of motion.

\section{Conclusion}

This study shows that in our setting twice daily PT sessions do not produce different results as daily PT sessions. It may be questioned whether multiple daily therapy sessions are needed as an in-hospital PT regimen in OA total knee patients. 


\section{Background}

Recent trends in the treatment of patients following orthopaedic surgery in the Netherlands encourage early discharge from hospitals, which implies increasing the intensity of treatment during the in-hospital phase. The strategy of choice involves mobilisation of the joints, starting on the day of surgery as well as shortening the bed rest phase and early ambulation. Physiotherapy treatment (PT) plays an important role in this strategy.

In 2000 a 'Joint Care' programme was implemented at the University Hospital Maastricht. This is a clinical pathway in which the activities of physicians, nurses, physical therapists and other staff inside as well as outside the hospital are coordinated to provide the best overall care for patients with a particular diagnosis or procedure ${ }^{1}$.

Clinical pathways are used in many institutions for patients undergoing total knee arthroplasty (TKA) and total hip arthroplasty (THA). These procedures lend themselves very well to a pathway approach, because the process of care is relatively standardized. Clinical pathways have been successful in reducing costs and length of stay in acute care hospitals, while not compromising patient outcomes ${ }^{1-7}$. The contents of the individual treatments are hospital-specific to create an optimal regimen of care tailored to that specific institution. The main goal in the clinical stage is to prepare patients for discharge from the hospital as soon as possible after their operation. Discharge depends primarily on restoring sufficient functional range of motion $(\mathrm{ROM})^{8}$.

Various authors ${ }^{9-12}$ have described aggressive rehabilitation schemes incorporating more than one PT treatment session per day in the clinical phase following total knee surgery. Aggressive rehabilitation is believed to be important in preventing postoperative contracture of the soft tissue and in gaining better flexion. Nevertheless evidence of effects of different exercise programs following TKA is limited ${ }^{13}$. Our current PT programme is based on these rehabilitation schemes, as well as on the outcome of a nonrandomised controlled trial ${ }^{14}$ comparing usual care with a 'Joint Care' clinical pathway. This trial found the length of hospital stay to be significantly shorter in the 'Joint Care' group.

The author concluded that this was mainly caused by the greater stimulation to exercise and the number of minutes of PT per day. Patients were not only stimulated by their physical therapists to exercise, but the nursing staff also stimulated the patients to exercise and perform daily life activities themselves. Based on this result we doubled the time spent on in-hospital PT from 20 minutes to 40 minutes per patient per day, thus increasing treatment intensity to fulfil one of the goals of the programme; shortening hospital stay to four days after surgery.

Patients are scheduled for discharge the fourth day after surgery if their passive flexion ROM exceeds $65^{\circ}$, when they are able to transfer from 
sitting to standing, walk independently, and are able to climb stairs, if necessary.

After two years of experience with the high intensity approach, the mean hospital stay was reduced to approximately four days after the operation. In 2003, however budget considerations meant that continuation of the extra PT sessions was being reconsidered. This led to a debate within the PT department whether or not the extra time investment should be maintained, i.e. whether the second daily visit was essential in reaching the short-term PT goals and whether having only one session of PT a day would lead to poorer range of motion (ROM) and function.

This resulted in the following research question. Does the intensity of PT treatment (once versus twice daily) in patients following TKA affect their short-term recovery, measured as range of motion?

To answer this question, a randomised controlled in-hospital trial was conducted in which a regimen consisting of four days with 40 minutes of PT a day was compared with one involving 20 minutes of PT a day.

\section{Methods}

The medical ethics committee of the Maastricht University Hospital and Maastricht University approved this prospective, randomised controlled trial.

The design of the trial is depicted in figure 1.

Fig 1: study design and outcome assessment.

T0

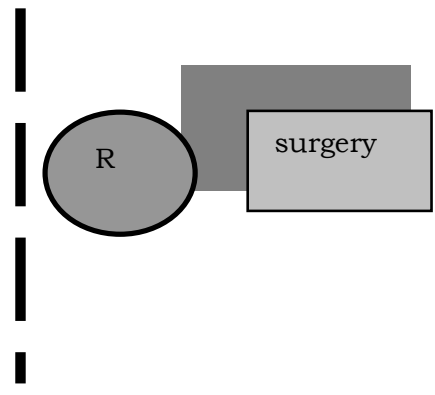

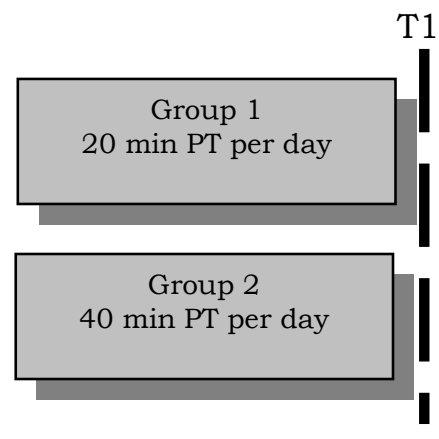

$\mathrm{T} 2$

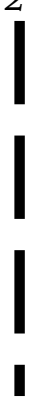

TO baseline assessment, one week before surgery,

T1 assessment 4 days after surgery,

T2 assessment 6 weeks after surgery,

T3 assessment 3 months after surgery

$\mathrm{R}=$ randomisation

\section{Subjects}

A consecutive series of patients who received a primary TKA at the University Hospital Maastricht, the Netherlands, was invited to participate 
between 1 January and 1 June, 2004. Subjects were considered eligible for the study if they were scheduled in the 'Joint Care' programme and signed an informed consent form.

Patients undergoing knee revision surgery were excluded as were patients over 85 years of age, patients with co-morbidity influencing gait and patients who did not understand or speak Dutch.

\section{Randomisation}

An independent research assistant performed concealed randomization using a computer generated randomisation schedule in which patients were allocated according to a weekly treatment regimen. All patients are admitted into hospital on Monday evening, surgery is on Tuesdays and hospital leave is planned the next Saturday. By randomising clusters of treatment weeks instead of individual randomisation we made sure that patients within the same treatment week all received the same postoperative treatment regime, thus avoiding contamination, which might occur when patients receive different regimes in the same week of admission at the same ward.

Information on the particular treatment intensity was given on the day of surgery. In this way we prevented foreknowledge of treatment assignment and thus shielded those who enrolled participants from being influenced by this knowledge because enrolment into the study was conducted two weeks before surgery.

\section{Interventions}

\section{Clinical phase}

During the in-hospital phase daily PT was administered to all participating patients. Patients in the experimental group were treated twice daily (totalling 40 minutes per day), patients in the control group once daily (20 minutes per day). The treatment content was similar in both groups and consisted of active and passive mobilisation of the knee, strengthening of the quadriceps muscle and functional exercises including transfers from a supine position to sitting and from sitting to standing, walking and stair climbing. As a rule, patients left hospital after the morning PT sessions on the fourth day after surgery, leading to a total number of PT sessions in the experimental group of seven sessions compared to four sessions in the control group.

A detailed description of the treatment protocol following total knee surgery is available at the website of the Physiotherapy department of the Maastricht University Hospital ${ }^{15}$

We divided the treating physical therapists into two groups. One group treated all patients in the morning, while the optional second treatment sessions were administered by other physical therapists, leaving the group of therapists who treated the patients in the morning sessions unaware whether or not patients were receiving a second PT session. 
Therapist received weekly work schedules. During the study period therapist shifted from morning to afternoon groups randomly over treatment weeks. These changes were introduced to balance the work load of the participating therapist rather than for study purposes.

Hospital discharge followed when patients showed wound healing, they were ambulant using a walking frame or crutches and passive flexion $\mathrm{ROM}$ of the knee was above 65 degrees.

\section{Outcome measures}

Primary outcome measure in this study was passive flexion ROM.

Secondary outcome measures were active ROM and passive extension ROM, functional status, length of stay (LOS) after surgery, pain over the last 24 hours (using an 11 point scale), satisfaction with treatment (using an 11 point scale from totally dissatisfied to completely satisfied) ${ }^{16}$, satisfaction with the intermediate treatment results and global perceived effect.

ROM was measured actively as well as passively using a large goniometer following the method described by Brosseau ${ }^{17}$. Intratester reliability for knee flexion is 0.99 , for active extension 0.97 . Criterion validity for knee flexion is 0.98 , for extension $0.42^{17}$.

Functional status was measured using two scales, the disease specific Western Ontario and McMaster University Osteoarthritis index (WOMAC) $)^{18}$ and the joint specific Knee Society Scale (KSS) ${ }^{19}$.

The WOMAC is a disease specific questionnaire developed specifically for people with osteoarthritis of the hip and knee. The WOMAC consists of 24 items which probe 3 dimensions; pain (5 items), stiffness (2 items), and functional difficulty (17 items). Scale sum scores were standardised (0-100), with high values indicating less pain or better physical functioning ${ }^{20}$.

The WOMAC questionnaire is well recognized for its good validity, reliability and responsiveness. We used the Dutch version of WOMAC ${ }^{20}$.

The KSS is concise and easy to use. It represents a clear attempt to separate knee function from overall patient functional status. Bach et $\mathrm{al}^{21}$ reported that reproducibility of the knee score is poor whilst the function score shows good reproducibility. The construct validity of the KSS is good 22 .

At follow-up, patients were asked to judge the effect of the surgery on a 7-point Likert scale from 'worse than ever'status to 'completely recovered'23.

The primary effect measurement was scheduled for the fourth day after surgery, while follow-up measurements were scheduled at 6 weeks and 3 months after surgery. 
Table 1 shows the timing and measurement tools used. The outcome assessor was blinded with regard to the clinical intervention regime administered.

Table 1 outcome measures and timing ROM= Range of Motion

\begin{tabular}{lllll}
\hline & T0 & T1 & T2 & T3 \\
ROM & X & X & X & X \\
Functional status WOMAC & $\mathrm{X}$ & & $\mathrm{X}$ & $\mathrm{X}$ \\
Functional status KSS & $\mathrm{X}$ & $\mathrm{X}$ & $\mathrm{X}$ & $\mathrm{X}$ \\
Length of stay & & $\mathrm{X}$ & & \\
Pain & $\mathrm{X}$ & $\mathrm{X}$ & $\mathrm{X}$ & $\mathrm{X}$ \\
Treatment satisfaction & & $\mathrm{X}$ & $\mathrm{X}$ & $\mathrm{X}$ \\
Satisfaction with intermediate results & $\mathrm{X}$ & $\mathrm{X}$ & $\mathrm{X}$ \\
Number of PT sessions & $\mathrm{X}$ & & \\
$\begin{array}{l}\text { Global perceived effect } \text { of total treatment by } \\
\text { the patient }\end{array}$ & $\mathrm{X}$ & $\mathrm{X}$ & $\mathrm{X}$ \\
\hline
\end{tabular}

T0, 14 days prior to surgery

T1, 4 days after surgery

T2, 6 weeks after surgery

T3, 3 months after surgery

We selected the KSS score as functional outcome on day four because several items in the WOMAC are not applicable during the in-hospital period. The WOMAC was assessed during the outpatient period because psychometric properties of the WOMAC are superior to the KSS.

\section{Sample Size}

A $10^{\circ}$ of passive ROM difference between groups was considered clinically important enough to eventually raise treatment frequency. The sample size needed to detect a significant difference $(2$-sided, $\alpha<$. 05) of at least $10^{\circ} \mathrm{ROM}$ between groups (primary outcome) with an assumed standard deviation (SD) of $10^{\circ}$ and a power of 0.8 , was 16 subjects per group. To compensate for a maximally acceptable $20 \%$ loss of subjects between baseline and follow-up, 20 subjects per group (40 in total) were required.

\section{Analysis}

Data were checked for completeness and normality. Statistical analysis were blinded and performed according to the 'intention-to-treat' principle. 
Descriptive statistics were calculated of both group statuses at baseline. Post treatment scores as well as differences between pre- and post treatment scores were compared with regard to all outcome measures. Group differences and 95\% confidence intervals (CI) were computed for all outcome measures. The scores on global perceived effect were dichotomized to examine the ratio of improved versus unimproved patients. Patients were scored as improved if they felt much better or had recovered completely. Student's t-test was used for continuous data to determine differences between the two treatment groups. Chi-square tests were used for analysis of categorical data and when continuous data were not normally distributed. All data were analysed using SPSS version $11.0^{24}$.

\section{Results}

Of the 55 subjects who were scheduled for surgery between January and June 2004, 43 were included in the study. 9 patients refused to participate and 3 were excluded because they had relevant comorbidities. Twenty-one patients were randomly assigned to the experimental group, 22 to the control group. 
Figure 2: Flow chart of subjects through the trial.

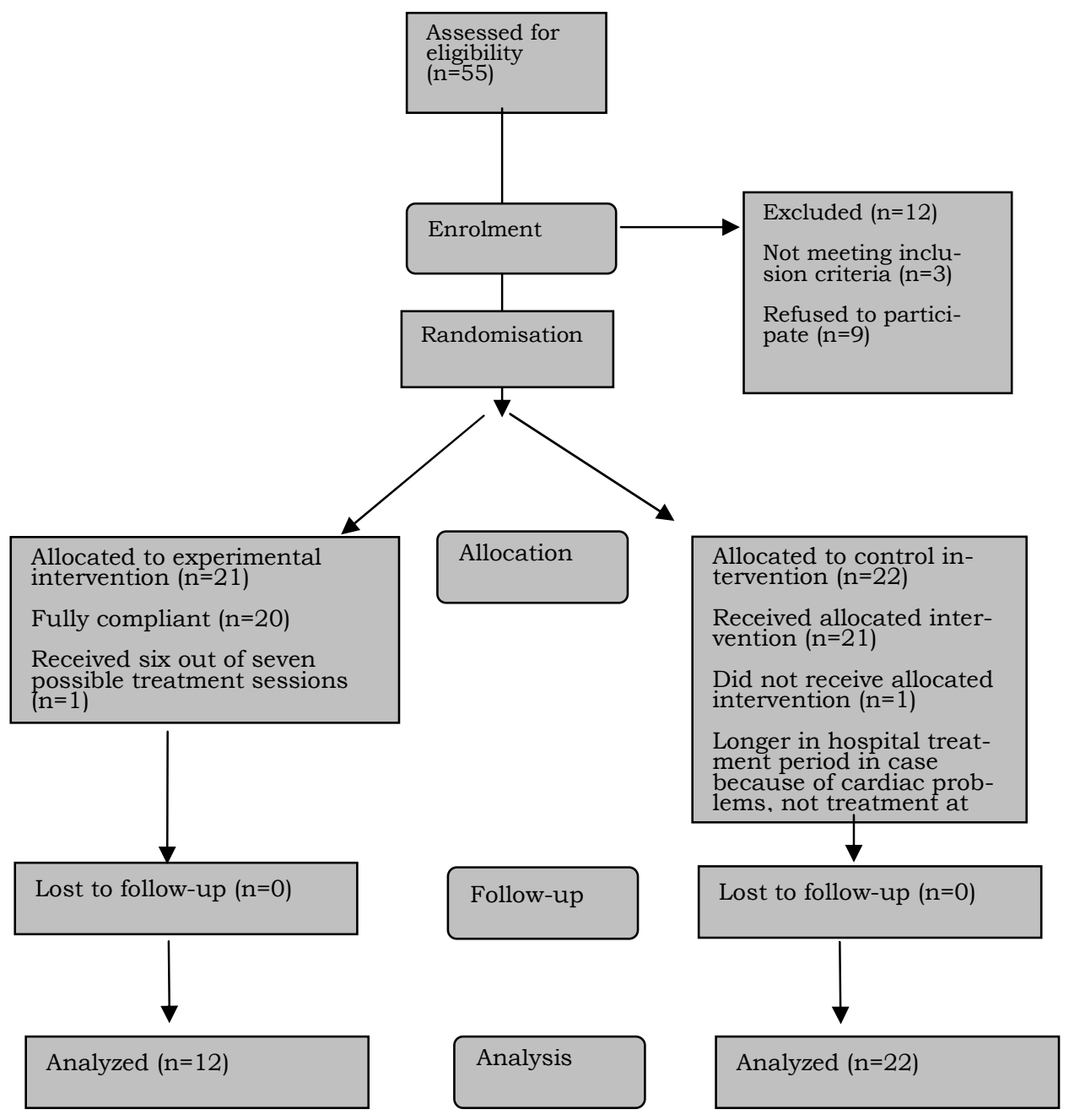

The baseline characteristics of the patients in the two groups were similar in terms of clinical and demographic characteristics. They also showed comparable levels of functional ability and Quality of Life (table 2). 
Table 2 patient's baseline characteristics

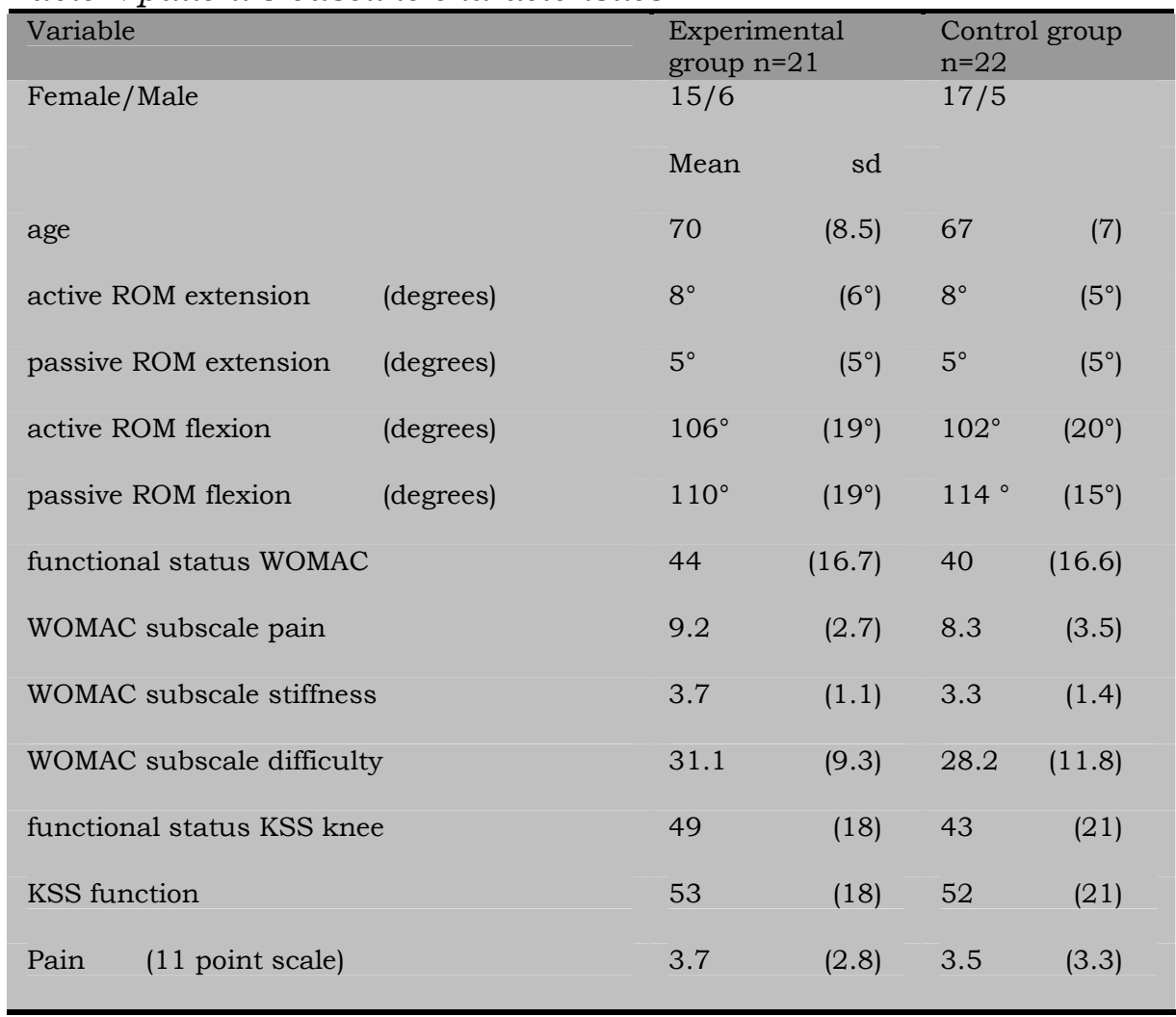

All 43 patients were assessed two weeks before surgery (T0) and four days after surgery (T1).

Six weeks after the operation (T2) 2 patients of the control group failed to attend the follow-up measurement, thus data from 41 patients were available for intention to treat analysis. All participants attended the follow-up measurements at 3 months.

Outcome: 4 days after surgery.

As can be seen from table 3 , there was no detectable difference in the primary outcome measure ROM between the experimental and control groups four days after surgery. 


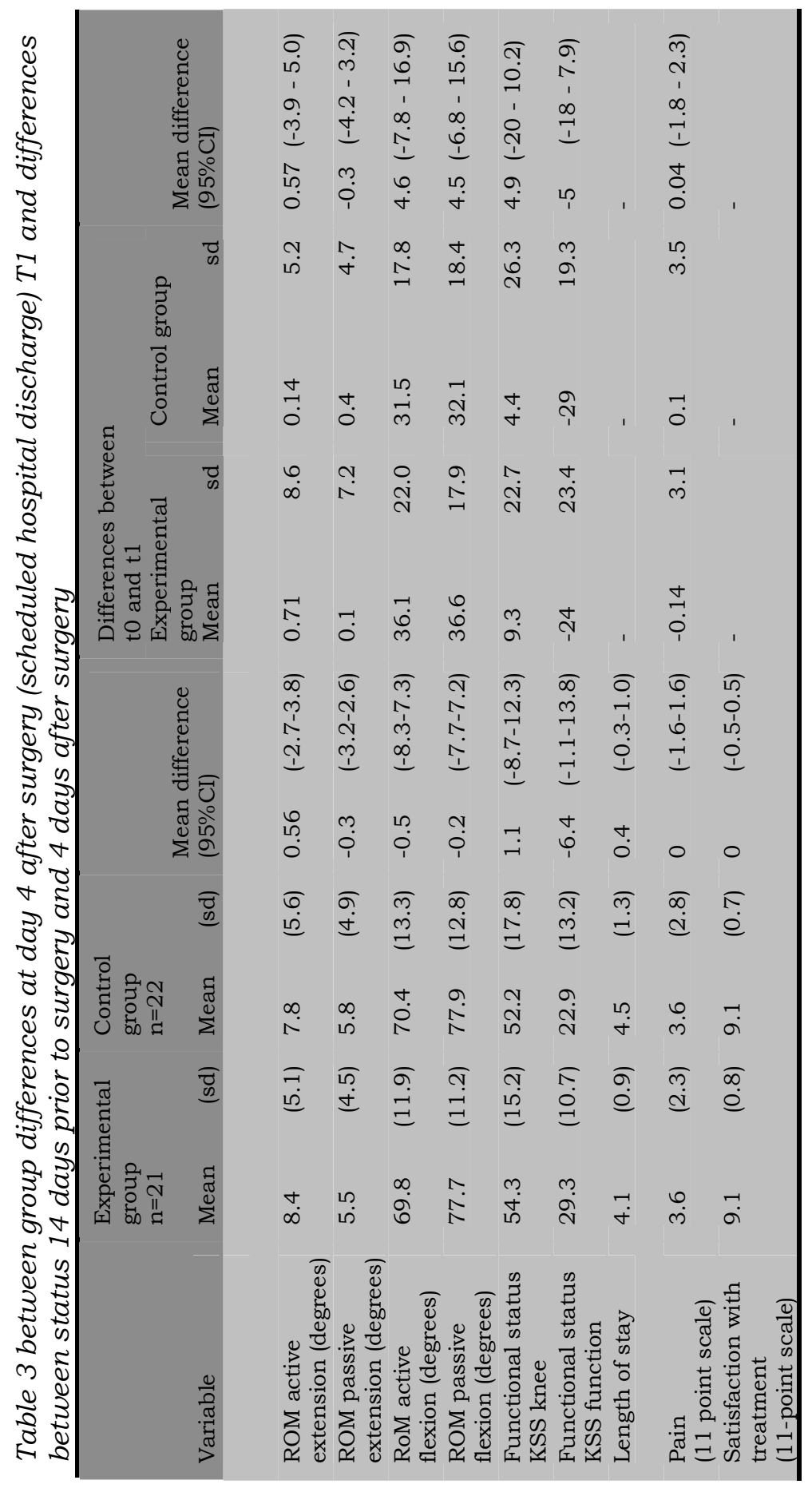




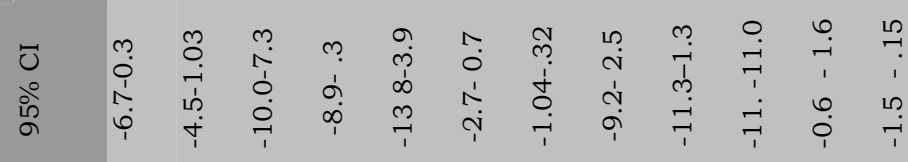

政莺

营

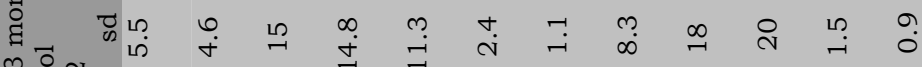

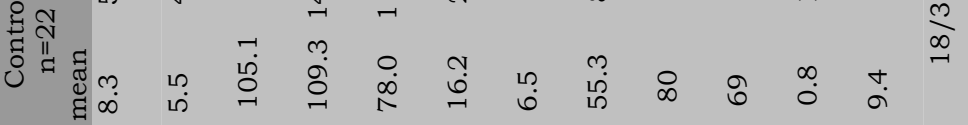

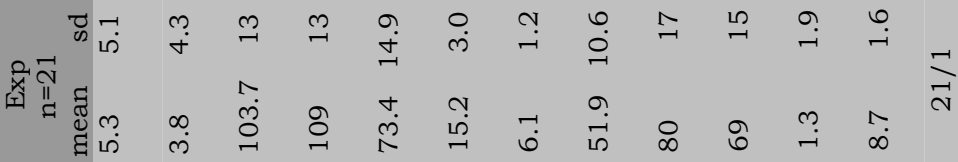

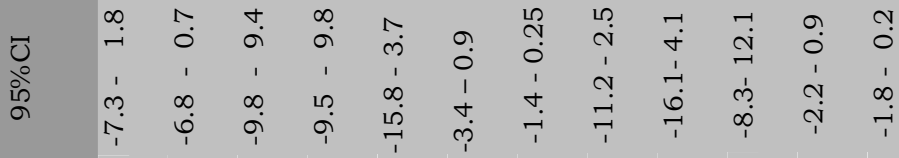

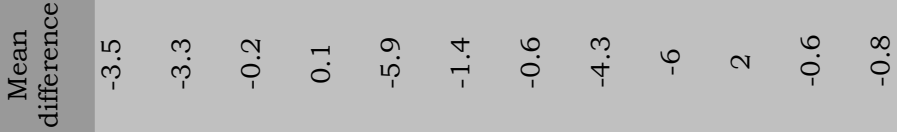

$\infty$

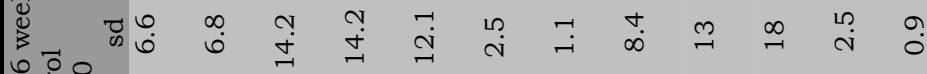

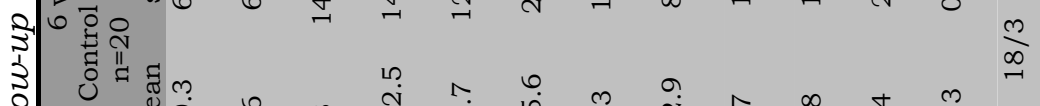

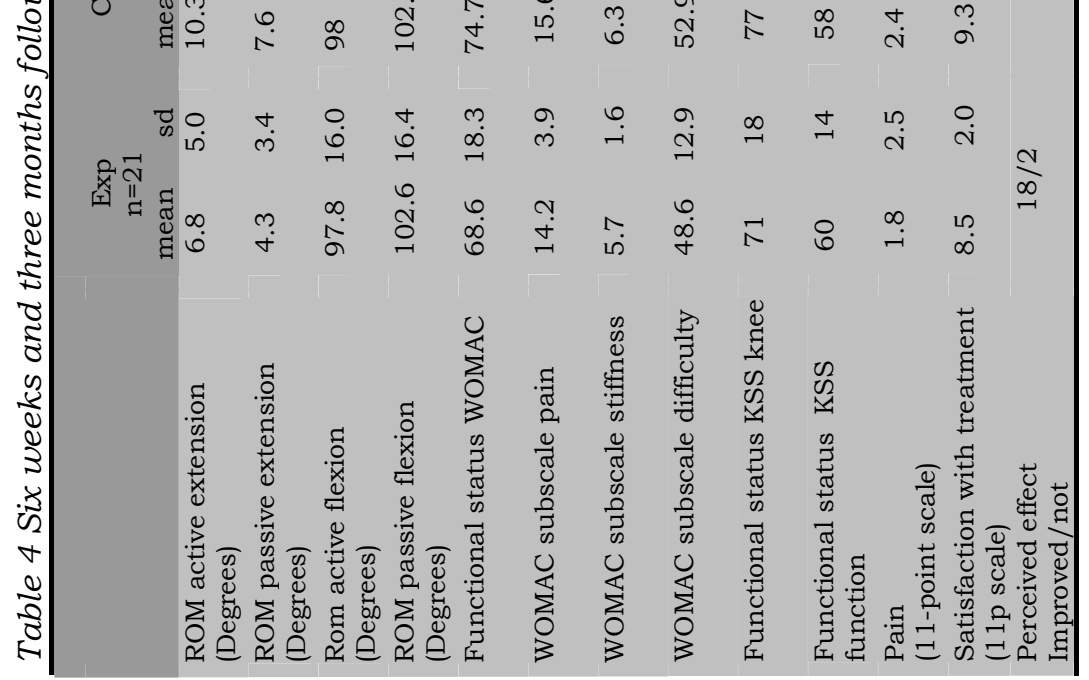

70. Chapter 5 
Patients in the control group all received 4 treatment sessions. Two patients in the experimental group received six of the seven possible treatment sessions. One of these patients did not feel well enough to participate in a second PT session on the second day after surgery. The other patient left the hospital on the third day after surgery. The surgeon approved his discharge since he had reached the short-term treatment goals and asked to return home for personal reasons. Length of stay was similar in both groups. One patient in the control group was transferred to the cardiology department because of cardiac complaints. He left the hospital 15 days after surgery.

Flexion contracture was about $8^{\circ}$ in both groups and active flexion ROM was about $70^{\circ}$

Functional status results on day four were also similar between groups.

Six-weeks and three-month's follow-up

There were no significant or clinically relevant differences in the secondary outcome measures at any of the other follow-up moments (table 4). Follow-up data were comparable to those at day four after surgery.

ROM in extension seemed to be a little better in the control group, as was satisfaction with the overall treatment. Recovery of flexion range of motion as well as functional status is similar in both groups.

\section{Discussion}

This trial did not detect any differences in the effects on any of the outcome parameters between regimens involving one or two physiotherapy sessions a day in the clinical phase following TKA.

Remarkably, patients seemed to be very satisfied with PT treatment, irrespective of the number of sessions.

Three months after surgery, the perceived effect of the total treatment was high, with 39 out of 43 patients stating that they had vastly improved or were completely cured.

De Jong ${ }^{14}$ reported similar numbers of PT sessions and comparable hospital stay (4.97 days) after surgery compared to our experimental group (4.1 days). De Jong did not report on ROM and functional capacity was measured using a scale of unknown reliability and validity.

Since we did not find any other studies on the effects of different PT treatment intensities following TKA, it is only possible to compare the results of the total group with those of other studies with known treatment intensity.

Most studies on rehabilitation after TKA have focussed on the use of continuous passive motion treatment.

Several recent studies ${ }^{95-27}$ incorporated high intensity basic PT rehabilitation programmes and describe outcomes comparable to our data. Two studies employed standardized PT treatments twice daily, whilst 
Kumar et al. reported 90 minutes to two hours of PT daily and Beaupre et al. did not standardize the treatment duration but reported on six treatment sessions during the first four days after surgery.

Three ${ }^{2526}$ studies described short-term results on range of motion.

The ROM we found four days after surgery is comparable to the values reported in these studies. The level of pain found in our study is in agreement with those reported by Bennett whereas the length of stay in Bennett's sample was longer (8.8 compared to 5 days), and KSS scores at 3-months follow-up were slightly better in our sample (mean KSS function score of 52 versus 69).

Our study only collected WOMAC data preoperative and at six-weeks and three-months follow- up. No WOMAC data were collected at hospital discharge because several items of the WOMAC score are not valid during hospital stay, items like getting in and out of a car, shopping, getting in and out of a bath and doing domestic chores are not applicable in this phase.

\section{Study limitations}

We chose cluster randomization of treatment weeks over individual randomization.

By clustering in treatment weeks we made sure that all patients operated in a week were receiving the same amount of daily treatment sessions. We believe that this led to less bias than introduced by contamination in individual randomization.

Patients receive PT treatment in the 'living room' of the orthopaedic ward in which they stay during daytime. We believe contamination is likely to occur when one patient would receive a second treatment in the afternoon whilst the patient sitting next to him/her is not. Conversation is overheard and just seeing treatment might encourage the patient to exercise them self, therewith diminishing contrast between both groups.

Though randomizing in clusters theoretically might inflict bias, we believed in this case it does not have a lot of influence.

It is accepted that clustering may result in $\mathrm{P}$ values and confidence intervals which are sufficiently biased to have a major effect if any of the following are true: the cluster size is large, the number of clusters is small, or the intra-cluster correlation coefficient is large 28 . Our study contained 22 clusters, cluster sizes were small with a mean of two patients per cluster and intracluster correlation was very small, (ICC=0,171), leading up to a design effect of $1.171^{29}$.

In combination with the fact that clusters were merely different weeks in a four months period in the same hospital with the same staff etc. makes us believe that clustering did not introduce a major bias in our study.

We chose passive flexion ROM as primary outcome measure because passive ROM is the only measure of function involved in hospital dis- 
charge in our situation. Therefore we based our sample size calculation on between group differences in passive flexion ROM.

When designing the study the therapists and orthopaedic surgeons involved discussed what effect would justify the effort of extra PT sessions daily. We had no information on possible effect sizes or minimally important clinical differences out of the literature, since we are unaware of a single similar study in this field. However we had some information on the effects of CPM in this same setting ${ }^{30}$. In this study we found an 8 degrees difference in flexion ROM when using CPM in the in hospital phase after total knee arthroplasty.

We agreed on 10 degrees between group differences to be a result worth the extra PT effort. Although we did not detect a between group difference at all, one may state that our study was designed to find a large difference. Sample size was calculated to find this effect and therefore may be too small to detect smaller differences. Our study is underpowered to detect significantly smaller between group differences.

For example, to detect a 5 degree difference sample size would a sample size of over 120 participants.

Although our study could not detect differences between groups receiving PT once or twice daily, this does not mean that individual patients might not benefit from more intensive PT.

One of the primary issues in treating patients with TKA is to identify those patients that may require more intensive rehabilitation. Unfortunately, secondary analysis yielded no variables at baseline that predicted short-term postoperative recovery.

Different therapists treated patients in the morning and afternoon sessions as well as over different treatment days. This is a common procedure in our hospital which in itself might increase treatment variation. However, treatments were standardized and applied following a protocol.

In this study, PT treatment was standardized in terms of content as well as duration of the treatment sessions. Although almost every study in this field has done the same, one might question whether this is the appropriate way to handle patient care. It might be wiser to set treatment goals per day.

As soon as the target for that day has been achieved no further treatment might be necessary. This would mean that the number of treatments can be individualized according to the goals set for each day.

Although blinding therapists is very difficult, we attempted to blind at least the physical therapists attending the morning session. Blinding was not successful in every case, because some patients gave information about the second session during the morning sessions.

Participating physical therapists stated that not knowing whether a patient would receive a second treatment session probably raised their awareness about achieving clinical goals during the morning sessions. Although they were aware that this attitude might bias study results, their attitude towards giving optimal patient care overruled the urge to 
follow the treatment protocol. In this sense one might say that treating patients once daily is just as good as twice daily if the physical therapists are more focussed.

\section{Conclusions}

This study shows that in our setting twice daily PT sessions do not produce different results as daily PT sessions. It may be questioned whether multiple daily therapy sessions are needed as an in-hospital PT regimen in OA total knee patients.

\section{Competing interests}

The authors declare that they have no competing interests.

\section{Authors' contributions}

AFL participated in the design of the study, exercise intervention, assessments and follow-ups, statistical analysis and writing. YHFC, EMHW, MJAvS and RAdB participated in the study design and the interpretation of the data, and critically revised the article. RGTG and PAvdB participated in the interpretation of the data and critically revised the article. All authors read and approved the final manuscript.

\section{Acknowledgements}

We thank all our co workers of the Orthopaedic and Physiotherapy department for their support during the trial. We thank all the patients who participated in the study for their valuable input. 


\section{References}

1. Macario A, Horne M, Goodman S, Vitez T, Dexter F, Heinen R, et al. The effect of a perioperative clinical pathway for knee replacement surgery on hospital costs. Anesth Analg 1998;86(5):978-84.

2. Pennington JM, Jones DP, McIntyre S. Clinical pathways in total knee arthroplasty: a New Zealand experience. J Orthop Surg (Hong Kong) 2003;11(2):166-73.

3. Mabrey JD, Toohey JS, Armstrong DA, Lavery L, Wammack LA. Clinical pathway management of total knee arthroplasty. Clin Orthop Relat Res 1997(345):125-33.

4. Harlan K, Meiring A. Total knee arthroplasty clinical pathway. Hosp Case Manag 1999;7(3):49-53.

5. Healy WL, Iorio R, Ko J, Appleby D, Lemos DW. Impact of cost reduction programs on short-term patient outcome and hospital cost of total knee arthroplasty. J Bone Joint Surg Am 2002;84-A(3):348-53.

6. Pearson S, Moraw I, Maddern GJ. Clinical pathway management of total knee arthroplasty: a retrospective comparative study. Aust $N Z \mathrm{~J}$ Surg 2000;70(5):351-4.

7. Kim S, Losina E, Solomon DH, Wright J, Katz JN. Effectiveness of clinical pathways for total knee and total hip arthroplasty: literature review. $J$ Arthroplasty 2003;18(1):69-74.

8. Oldmeadow LB, McBurney H, Robertson VJ. Hospital stay and discharge outcomes after knee arthroplasty: implications for physiotherapy practice. Aust J Physiother 2002;48(2):117-21.

9. Bennett LA, Brearley SC, Hart JA, Bailey MJ. A comparison of 2 continuous passive motion protocols after total knee arthroplasty: a controlled and randomized study. J Arthroplasty 2005;20(2):225-33.

10. Davies DM, Johnston DW, Beaupre LA, Lier DA. Effect of adjunctive range-of-motion therapy after primary total knee arthroplasty on the use of health services after hospital discharge. Can J Surg 2003;46(1):30-6.

11. Mauerhan DR, Mokris JG, Ly A, Kiebzak GM. Relationship between length of stay and manipulation rate after total knee arthroplasty. $J$ Arthroplasty 1998;13(8):896-900.

12. Mokris JG, Smith SW, Anderson SE. Primary total knee arthroplasty using the Genesis Total Knee Arthroplasty System: 3- to 6-year follow-up study of 105 knees. J Arthroplasty 1997;12(1):91-8.

13. Roos EM. Effectiveness and practice variation of rehabilitation after joint replacement. Curr Opin Rheumatol 2003;15(2):160-2.

14. Jong de JA. Reactivatie van een THA patient volgens een oud'of een 'nieuw'protocol. MSc thesis, Maastricht University, Faculty of Health Sciences 1999.

15. http://www.azm.nl/professionals/overige+afdelingen/overzicht+overige+ afdelingen/fysiotherapie/onderzoek/treatmentprotocoltkpshortenglish.pdf

16. Bullens PH, van Loon CJ, de Waal Malefijt MC, Laan RF, Veth RP. Patient satisfaction after total knee arthroplasty: a comparison between subjective and objective outcome assessments. $J$ Arthroplasty $2001 ; 16(6): 740-7$. 
17. Brosseau L, Balmer S, Tousignant M, O'Sullivan JP, Goudreault C, Goudreault $\mathrm{M}$, et al. Intra- and intertester reliability and criterion validity of the parallelogram and universal goniometers for measuring maximum active knee flexion and extension of patients with knee restrictions. Arch Phys Med Rehabil 2001;82(3):396-402.

18. Bellamy N, Buchanan WW, Goldsmith $\mathrm{CH}$, Campbell J, Stit tLW. Validation study of the WOMAC: a health status instrument for measuring clinically important patient relevant outcomes following total hip or knee arthroplasties in osteoarthritis. $J$ Orthop Rheumatology 1988; 1:95-108.

19. Insall JN, Dorr LD, Scott RD, Scott WN. Rationale of the Knee Society clinical rating system. Clin Orthop Relat Res 1989(248):13-4.

20. Roorda LD, Jones CA, Waltz M, Lankhorst GJ, Bouter LM, van der Eijken $\mathrm{JW}$, et al. Satisfactory cross cultural equivalence of the Dutch WOMAC in patients with hip osteoarthritis waiting for arthroplasty. Ann Rheum Dis 2004;63(1):36-42.

21. Bach CM, Nogler M, Steingruber IE, Ogon M, Wimmer C, Gobel G, et al. Scoring systems in total knee arthroplasty. Clin Orthop Relat Res 2002(399):184-96.

22. Lingard EA, Katz JN, Wright RJ, Wright EA, Sledge CB. Validity and responsiveness of the Knee Society Clinical Rating System in comparison with the SF-36 and WOMAC. J Bone Joint Surg Am 2001;83-A(12):185664.

23. Guyatt GH, Townsend M, Berman LB, Keller JL. A comparison of Likert and visual analogue scales for measuring change in function. J Chronic Dis 1987;40(12):1129-33.

24. Norusis MJ. SPSS 9.0 guide tot data analyses. Chicago: SPSS Inc.1999.

25. Beaupre LA, Davies DM, Jones CA, Cinats JG. Exercise combined with continuous passive motion or slider board therapy compared with exercise only: a randomized controlled trial of patients following total knee arthroplasty. Phys Ther 2001;81(4):1029-37.

26. Kumar PJ, McPherson EJ, Dorr LD, Wan Z, Baldwin K. Rehabilitation after total knee arthroplasty: a comparison of 2 rehabilitation techniques. Clin Orthop Relat Res 1996(331):93-101.

27. MacDonald SJ, Bourne RB, Rorabeck CH, McCalden RW, Kramer J, Vaz M. Prospective randomized clinical trial of continuous passive motion after total knee arthroplasty. Clin Orthop Relat Res 2000(380):30-5.

28. Bland JM. Cluster randomised trials in the medical literature: two bibliometric surveys. BMC Med Res Methodol 2004;4:21.

29. Campbell MK, Elbourne DR, Altman DG. CONSORT statement: extension to cluster randomised trials. Bmj 2004;328(7441):702-8.

30. Lenssen AF, Bie de RA, Bulstra SK, Steijn van MJA. Continuous passive motion in rehabilitation following total knee arthroplasty: a Randomised controlled trial. Physical Therapy Reviews 2003;8(3):123-129. 
Effectiveness of prolonged use of continuous passive motion (CPM) as an adjunct to physiotherapy following total knee arthroplasty:

Design of a randomised controlled trial

\author{
AF Lenssen \\ YHF Crijns \\ EMH Waltjé \\ GM Roox \\ MJA van Steyn \\ RJT Geesink \\ PA van den Brandt \\ RA de Bie
}

BMC Musculoskeletal Disorders 2006, 7:15 


\section{Abstract}

\section{Background}

Adequate and intensive rehabilitation is an important requirement for successful Total Knee Arthroplasty. The primary focus of early rehabilitation is ambulation of patients and regaining range of motion in the knee.

Although research suggests that Continuous Passive Motion should be implemented in the first rehabilitation phase following surgery, there is substantial debate about the duration of each session and the total period of CPM application and. A Cochrane review on this topic concluded that short-term use of CPM leads to greater short-term range of motion. It also suggested, however, that future research should concentrate on the treatment period during which CPM should be administered.

\section{Methods}

In a randomized controlled trial we intend to investigate the efficacy of prolonged use of a continuous passive motion (CPM) device in the home situation as an adjunct to standardised physiotherapy. The experimental treatment is compared to standardised physiotherapy, in patients with osteoarthritis of the knee undergoing Total Knee Arthroplasty (TKA). Efficacy will be assessed in terms of faster improvements in range of motion and functional recovery.

Seventy patients with knee osteoarthritis undergoing TKA and experiencing early postoperative flexion impairment (less than $80^{\circ}$ of knee flexion at the time of discharge) will be randomised over two treatment groups, a usual care group and an experimental group

The experimental group will receive CPM + physiotherapy for 17 consecutive days after surgery, whereas the usual care group will receive the same treatment during the in-hospital phase (i.e. about four days), followed by physiotherapy alone (usual care) in the first two weeks after hospital discharge.

From 18 days to three months after discharge, both groups will receive standardised PT. The primary focus of rehabilitation will be functional recovery (e.g. ambulation) and regaining range of motion (RoM) in the knee.

\section{Discussion}

Because restricted knee RoM affects functional activities, knee RoM and knee function are regarded as the primary indicators of successful TKA.

Potential effects of the intervention under study include rapid return of knee flexion accompanied by earlier return to functional activities of daily life. If patients benefit significantly from prolonged CPM use, this treatment should be added to the standard PT treatment at home.

We expect the additional home CPM programme to be more effective than the usual physiotherapy programme, resulting in a difference in 
RoM of at least $5^{\circ}, 17$ days after surgery. This clinically important difference, with a possible flexion RoM of about $100^{\circ}$, is expected to lead to better functioning in activities of daily life, like walking, and earlier ability to cycle. These advantages should result in earlier and increasing independence. 


\section{Background}

With the ageing of the population, the prevalence of degenerative joint diseases is increasing. Reports show that over a one-year period, $25 \%$ of people over 55 years have a persistent episode of knee pain, of whom annually about one in six consult their general practitioner, in both the UK and the Netherlands.

The prevalence of painful disabling knee osteoarthritis in people over 55 years is $10 \%$, of whom one quarter is severely disabled. In all, over 300,000 Dutch residents currently suffer from knee osteoarthritis (OA). Total Knee Arthroplasty (TKA) is a common intervention that can enhance the quality of life for patients with knee OA. Over 7500 TKAs are performed in Dutch hospitals every year. In 2003, 160 TKAs were performed at the Maastricht University Hospital. Adequate and intensive rehabilitation is an important requirement for successful TKA. The primary focus of early rehabilitation is ambulation and regaining range of motion (RoM) in the knee. Because restricted knee RoM affects functional activities, knee RoM is regarded as one of the primary indicators of a successful TKA. Rapid return of knee flexion accompanied by earlier return to functional activities of daily life is one of the potential effects of the intervention we are proposing to study.

Although research findings favour the use of Continuous Passive Motion $(\mathrm{CPM})$ in the first rehabilitation phase following surgery ${ }^{1-4}$, there is substantial debate about the total period of CPM application and the duration of individual sessions. A Cochrane review ${ }^{1}$ on the topic concluded that short-term use of CPM leads to a more rapid recovery of range of motion. It also suggested, however, that future research should concentrate on the period during which CPM should be administered, and therefore called for research into longer-term use, involving long term follow-up.

Before the year 2000, discharge from the Maastricht University Hospital after TKA was scheduled approximately 14 days after surgery. Nowadays, most patients are discharged four days after surgery. Since the time spent in hospital following surgery has decreased, continuation of CPM after hospital discharge might be beneficial. Although CPM is increasingly being administered in the post clinical home situation in recent years and is beginning to become part of the usual care programme, proper research into the effectiveness of a prolonged use of CPM at home is still lacking ${ }^{12}$. The only study that has been reported ${ }^{5}$ compared CPM with physiotherapy (PT) as

a stand-alone therapy, whereas in the study presented here, CPM will be added to a standardised programme, adequately reflecting current practice.

This study is expected to provide new insights into the additional effects of CPM and to provide evidence to justify or refute its use. 
Currently, orthopaedic surgeons at the hospital and physiotherapists at the hospital and at home play an important role in the rehabilitation process for TKA patients. The proposal presented here involves the same health care professionals and the same treatment strategies currently in use, but one patient group will additionally receive CPM at home.

We expect the effect of CPM treatment to be a quicker restoration of RoM, resulting in improved ADL function during the first 3 months after surgery.

Knee flexion values of $95^{\circ}$ and $105^{\circ}$ are regarded as range of motion benchmarks ${ }^{6}$ in the functional recovery following CPM. While $95^{\circ}$ of knee flexion allows normal ADL function, $105^{\circ}$ of flexion gives a person the opportunity to ride a bicycle. This is of great advantage both in daily life (at least in the Netherlands) and in the rehabilitation from TKA surgery, because cycling allows patients to move the knee much more. We expect that prolonged use of CPM at home will allow patients to achieve these RoM benchmarks earlier in their recovery process.

About $50 \%$ of the patients undergoing one of the 160 TKAs performed annually at the Maastricht University Hospital have less than $80^{\circ}$ of flexion 4 days after surgery and therefore potentially meet the inclusion criteria of the proposed study.

If the study should find that patients benefit from home CPM, this treatment should be added to the standard PT treatment currently administered. No differences in direct costs of rehabilitation are expected from implementation of the study results.

\section{Objective}

Continuous passive motion (CPM) has proved to increase the amount of knee flexion for knee patients in the acute hospital setting (5-10 days). The primary purpose of the proposed randomised controlled trial is to establish whether there is additional long term benefit of continuing CPM after hospital discharge.

\section{Research question}

What is the effect on range of motion and functional status of prolonged use of a continuous passive motion device at home in addition to physiotherapy, compared to physiotherapy alone, in patients with limited flexion range of motion (less than $80^{\circ}$ ) of the knee at discharge from the hospital following total knee arthroplasty? 


\section{Methods/Design}

\section{Study design}

The proposed study is a randomised controlled trial, with blinded treatment allocation, assessment and analysis, in which 70 patients undergoing a scheduled unilateral TKA will be evaluated to assess the added value of CPM at home, using function and mobility as the main outcomes.

\section{Participants}

We intend to include 70 patients in the study, who must be eligible for unilateral primary TKA as a result of osteoarthritis of the knee. Patients will be included if they;

have less than $80^{\circ}$ of flexion range of motion 4 days after surgery, are able to understand and speak Dutch, are not suffering from mental disabilities and are resident inside the 'Maastricht heuvelland' region. Patients will be excluded if they need to stay in hospital for more than 5 days after surgery or show relevant co-morbidity influencing mobility (e.g. claudicatio, other prosthesis) or are operated upon using minimally invasive surgery.

Eligible patients will be contacted one week before planned surgery, and will be randomised into two groups after signing an informed consent form, on the day they are discharged from hospital. Patients will thus have 10 days in which to consider participation.

Blocked and concealed randomisation with a block size of 4 will ensure equal distribution of patients over the two treatment groups. Groups will be prestratified on preoperative flexion mobility of the knee.

\section{Interventions}

During the in-hospital period, all patients will receive a standardised PT programme, involving 20 minutes of PT per day for four days. During the first two weeks after discharge, patients in the experimental group will receive CPM for 4 hours a day in addition to regular PT treatment. Patients in the control group will receive only regular PT treatment (usual care).

From day 18 onward, all patients will receive regular PT treatment until 3 months after surgery, if indicated.

The post-clinical PT will be standardised in terms of treatment objectives. All patients will receive treatment consisting of active and passive mobilisation of the knee joint, active strengthening of the $\mathrm{m}$. quadriceps, and training of ADL functions (gait, sit to stand and stair climbing). 


\section{Outcome assessment}

After baseline variables have been collected, outcome measures will be assessed at 17 days, 6 weeks and 3 months after surgery, during normal routine assessments at the orthopaedic clinic (table 1). The outcome assessor will be blinded.

Table 1: timing of the outcome assessment

\begin{tabular}{|c|c|c|c|c|c|}
\hline & $\begin{array}{l}\text { T0 } \\
\text { Pre- } \\
\text { operative }\end{array}$ & $\begin{array}{l}\text { T1 } \\
\text { Clinical } \\
\text { phase }\end{array}$ & $\begin{array}{l}\text { T2 } \\
17 \text { days } \\
\text { after sur- } \\
\text { gery }\end{array}$ & $\begin{array}{l}\text { T3 } \\
6 \text { weeks } \\
\text { after surgery }\end{array}$ & $\begin{array}{l}\text { T4 } \\
12 \text { weeks } \\
\text { after sur- } \\
\text { gery }\end{array}$ \\
\hline Range of motion & $\mathrm{X}$ & $\mathrm{X}$ & $\mathrm{X}$ & $\mathrm{X}$ & $\mathrm{X}$ \\
\hline Knee Society Score & $\mathrm{X}$ & $\mathrm{X}$ & $\mathrm{X}$ & $\mathrm{X}$ & $\mathrm{X}$ \\
\hline WOMAC & $\mathrm{X}$ & & $\mathrm{X}$ & $\mathrm{X}$ & $\mathrm{X}$ \\
\hline Perceived effect & & & $\mathrm{X}$ & $\mathrm{X}$ & $\mathrm{X}$ \\
\hline Pain medication & & $\mathrm{X}$ & $\mathrm{X}$ & $\mathrm{X}$ & $\mathrm{X}$ \\
\hline $\begin{array}{l}\text { Satisfaction with treat- } \\
\text { ment }\end{array}$ & & & $\mathrm{X}$ & $\mathrm{X}$ & $\mathrm{X}$ \\
\hline Satisfaction with results & & & $\mathrm{X}$ & $\mathrm{X}$ & $\mathrm{X}$ \\
\hline Compliance & & & $\mathrm{X}$ & & \\
\hline $\begin{array}{l}\text { Quantity, duration and } \\
\text { kind of treatment }\end{array}$ & & & $\mathrm{X}$ & $\mathrm{X}$ & $\mathrm{X}$ \\
\hline
\end{tabular}

Primary outcome measures are:

1. Functional status, using the WOMAC function score ${ }^{78}$ and the Knee Society Score'; and

2. Range of motion, assessed with a long-arm goniometer ${ }^{10}$.

Secondary outcome measures are:
A. perceived effect, using a 7-point Likert scale;
B. postoperative medication use (amount; type will be stan- dardised);
C. satisfaction with treatment, on an 11-point Likert scale;
D. satisfaction with treatment result, on an 11-point Likert scale;
E. adherence to treatment protocols and use of CPM (in hours), measured with a patient diary;
F. quantity, duration and nature of PT intervention. 
The study design is depicted in figure 1 .

Figure 1: Study design.

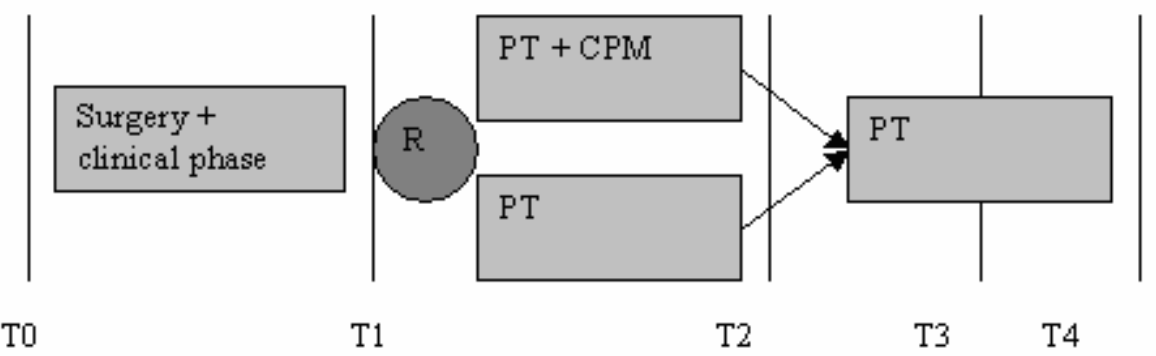

T0: one week before surgery

T1: 4 days after surgery,

T2: 17 days after surgery

T3: 6 weeks after surgery

T4: 3 months after surgery.

The first primary endpoint of the study is on day 17 after surgery, which is when the experimental treatment stops and short-term effects are measured.

The second endpoint is at 3 months after surgery. Table 1 shows the timing of the outcome assessment

\section{Power analysis}

We assume a difference of more than $5^{\circ}$ of knee flexion mobility (SD $8^{\circ}$ ) at the end of the CPM application to be clinically relevant. With an alpha of 0.05 , and a power of

$80 \%$, we will need 28 patients per group to prove this.

\section{Data analysis}

Data will be stored and analysed with SPSS-12.0 After checks for missing values and for normality of the data, regression techniques will be applied by a blinded analyst using the 'intention-to-treat' principle. Primary and secondary outcome measures will be reported for the inhospital and home situations, and for 6-week and 3-month follow-up. The primary research question will be tested using Student's t-tests with a $\mathrm{p}$-value of 0.05 being regarded as significant.

\section{Discussion}

\section{Dissemination}

The results of this trial are expected to provide evidence and clinically relevant information of direct use to professionals working in the field of rehabilitation after TKA (Orthopaedic surgeons, general practitioners, 
physical therapists, occupational therapists and nurses). If the additional use of CPM proves efficacious, CPM should be administered to the target population. Therefore, health insurance companies and public health services should take note of the results and implement the findings in their activities.

The results of the research will be disseminated by means of publications in peer-reviewed scientific journals, by updating the Dutch arthrosis guidelines for GPs and physical therapists, by publication of a $\mathrm{PhD}$ thesis and by means of lectures and presentations at conferences.

The relevant parties that can assure proper dissemination of the research findings are to a large extent represented in the project group. The orthopaedic surgeons are involved in drawing up the osteoarthritis guidelines, the research physical therapist and the project leader are responsible for the osteoarthritis guidelines for physiotherapists and the $\mathrm{PhD}$ student and promotion team are responsible for the scientific dissemination of the results.

The patients with osteoarthritis receiving TKA surgery will receive updated information through an updated leaflet, made available at the hospital.

\section{Ethics}

Patients will be asked to participate in the study one week prior to the planned surgery date. They will receive verbal and written information on the study. After doing so, they will have a 10-day period in which to decide whether or not to participate. Patients will be informed about the option to end their participation in the study at any time.

An independent physician will be appointed to whom patients can turn if they have any questions, etc. Written consent will be asked prior to participation. All data will be collected by a research assistant and will be stored in a database. The data will be anonymised and coded so third parties will not be able to link data to specific patients, thus ensuring complete confidentiality of data of individual patients. After conclusion of the study, all participants will be informed of the outcome.

\section{Risks of participation}

We believe there is very little additional risk for the participants included in the CPM treatment group. All patients will be familiar with the use of CPM device at the time they are discharged from the hospital. Nevertheless we have taken out supplementary insurance for all participants in the study. 


\section{References}

1. Milne S, Brosseau L, Robinson V, Noel MJ, Davis J, Drouin H, et al. Continuous passive motion following total knee arthroplasty. Cochrane Database Syst Rev 2003(2): CD004260.

2. Lenssen AF, Koke AJA, Bie de RA, Geesink RJT. Continuous passive motion in rehabilitation following total knee arthroplasty; short and long term effects on range of motion. Physical Therapy reviews 2003;8(3):113-121.

3. Lenssen AF, Köke AJA, De Bie RA. Effectiviteit van continuous passive motion in de klinische nabehandeling van patiënten na een totale knie prothese: een systematische review. Nederlands Tijdschrift voor Fysiotherapie 2001; dec. 2001(6):152-158.

4. Lenssen AF, Bie de RA, Bulstra SK, Steijn van MJA. Continuous passive motion in rehabilitation following total knee arthroplasty: a Randomised controlled trial. Physical Therapy Reviews 2003; 8(3):123-129.

5. Worland RL, Arredondo J, Angles F, Lopez-Jimenez F, Jessup DE. Home continuous passive motion machine versus professional physical therapy following total knee replacement. $J$ Arthroplasty 1998; 13(7):784-7.

6. Miner AL, Lingard EA, Wright EA, Sledge CB, Katz JN. Knee range of motion after total knee arthroplasty: how important is this as an outcome measure? J Arthroplasty 2003; 18(3):286-94.

7. Bellamy N, Buchanan WW, Goldsmith $\mathrm{CH}$, Campbell J, Stitt LW. Validation study of WOMAC: a health status instrument for measuring clinically important patient relevant outcomes to antirheumatic drug therapy in patients with osteoarthritis of the hip or knee. J Rheumatol 1988;15(12):1833-40.

8. Roorda LD, Jones CA, Waltz M, Lankhorst GJ, Bouter LM, van der Eijken JW, et al. Satisfactory cross cultural equivalence of the Dutch WOMAC in patients with hip osteoarthritis waiting for arthroplasty. Ann Rheum Dis 2004; 63(1):36-42.

9. Insall JN, Dorr LD, Scott RD, Scott WN. Rationale of the Knee Society clinical rating system. Clin Orthop Relat Res 1989(248):13-4.

10. Brosseau L, Balmer S, Tousignant M, O'Sullivan JP, Goudreault C, Goudreault $\mathrm{M}$, et al. Intra- and intertester reliability and criterion validity of the parallelogram and universal goniometers for measuring maximum active knee flexion and extension of patients with knee restrictions. Arch Phys Med Rehabil 2001;82(3):396-402. 


\title{
7
}

Effectiveness of prolonged use of continuous passive motion (CPM), as an adjunct to physiotherapy, after total knee arthroplasty.

\author{
AF Lenssen \\ MJA van Steyn \\ YHF Crijns \\ EMH Waltjé \\ GM Roox \\ RJT Geesink \\ PA van den Brandt \\ RA De Bie
}




\section{Abstract}

\section{Background}

Adequate and intensive rehabilitation is an important requirement for successful total knee arthroplasty. The primary focus of early rehabilitation is ambulation and regaining range of motion in the knee. Although research suggests that Continuous Passive Motion (CPM) should be implemented in the first rehabilitation phase after surgery, there is substantial debate about the duration of each session and the total period of CPM application. A Cochrane review on this topic concluded that short-term use of CPM leads to greater short-term range of motion. It also suggested, however, that future research should concentrate on the treatment period during which CPM should be administered.

\section{Methods}

In a randomised controlled trial we investigated the effectiveness of prolonged CPM use in the home situation as an adjunct to standardised PT. Experimental treatment was compared with standardised physiotherapy (PT) in patients with osteoarthritis (OA) of the knee undergoing Total Knee Arthroplasty (TKA). Efficacy was assessed in terms of faster improvements in range of motion (RoM) and functional recovery, measured at the end of the active treatment period, 17 days after surgery.

Sixty patients with knee osteoarthritis undergoing TKA and experiencing early postoperative flexion impairment (less than $80^{\circ}$ of knee RoM at the time of discharge) were randomised over two treatment groups. The experimental group received CPM + PT for 17 consecutive days after surgery, whereas the usual care group received the same treatment during the in-hospital phase (i.e. about four days), followed by PT alone (usual care) in the first two weeks after hospital discharge.

From 18 days to three months after surgery, both groups received standardised PT. The primary focus of rehabilitation was functional recovery (e.g. ambulation) and regaining RoM in the knee.

\section{Results}

Prolonged use of CPM improved short-term RoM in patients with limited RoM at the time of discharge after total knee arthroplasty when added to a semi-standard PT programme. Assessment at 6 weeks and three months after surgery found no long-term effects of this intervention Neither did we detect functional benefits of the improved RoM at any of the outcome assessments.

\section{Conclusion}

Although results indicate that prolonged CPM use has a short-term effect on RoM, routine use of prolonged CPM in patients with limited RoM at hospital discharge should be reconsidered, since neither long-term effects nor transfer to better functional performance was detected. 


\section{Background}

With the ageing of the population, the prevalence of degenerative joint diseases is increasing. Reports show that over a one-year period, $25 \%$ of people over 55 years have a persistent episode of knee pain, of whom annually about one in six consult their general practitioner, in both the UK and the Netherlands ${ }^{1}$. The prevalence of painful disabling knee osteoarthritis in people over 55 years is $10 \%^{2}$, of whom one quarter are severely disabled. In all, over 300,000 Dutch residents currently suffer from knee osteoarthritis (OA). Total Knee Arthroplasty (TKA) is a common intervention that can enhance the quality of life for patients with knee OA. Over 7500 TKAs are performed in Dutch hospitals every year. In 2004, more than 160 TKAs were performed at the Maastricht University Hospital.

Adequate and intensive rehabilitation is an important requirement for successful TKA. The primary focus of early rehabilitation is to prepare patients for discharge from the hospital as soon as possible after their operation. Because restricted knee range of motion (RoM) affects functional activities, knee RoM is regarded as one of the primary indicators of a successful TKA. Rapid return of knee RoM accompanied by earlier return to functional activities of daily life was one of the potential effects of the intervention applied in this study.

Continuous passive motion (CPM) is an external motorised device which enables a joint to move passively throughout a preset arc of motion. Robert Salter introduced the biological concept of CPM in the early 1980 s $^{3-6}$. He demonstrated in rabbit knees that CPM enhanced cartilage healing and regeneration compared to prolonged articular rest. Coutts et al 7 first initiated CPM use immediately after TKA. Their rationale was based on Salter's research and the postulate that CPM enhanced collagen tissue healing with better fibre orientation, avoiding cross-linking and thus generating better movement restoration.

CPM has been widely used as an adjunct to physiotherapy (PT) after TKA for the past two decades. However, there is still controversy as to whether it is useful. Various authors recommend $\mathrm{CPM}^{7-15}$, whereas others ${ }^{16-23}$ have found it to be of little value in the rehabilitation of the knee after TKA.

Although several systematic reviews favour the use of CPM in the first rehabilitation phase after surgery ${ }^{24-26}$, there still is substantial debate about the total period of CPM application and the duration of individual sessions. A Cochrane review ${ }^{24}$ on the topic concluded that use of CPM combined with PT offers beneficial results compared to PT alone in the short-term rehabilitation after TKA. It also suggested, however, that more research was required to assess the differences in CPM effectiveness with different characteristics of application, such as total duration of treatment and intensity of CPM interventions.

Most studies have evaluated effects during the acute in-hospital period. Before the year 2000, discharge from the Maastricht University Hospital after TKA was scheduled approximately 14 days after surgery. Nowa- 
days, most patients are discharged four days after surgery. Since the time spent in hospital after surgery has decreased, continuation of CPM after hospital discharge might be beneficial. Although CPM is now increasingly being administered in the post clinical home situation and is beginning to become part of the usual care programme, proper research into the effectiveness of a prolonged use of CPM at home is still lacking 2425 . The only study that has been reported ${ }^{20}$ compared CPM with PT as a stand-alone therapy, whereas in the study presented here, CPM was added to a standardised programme, adequately reflecting current practice, as orthopaedic surgeons at the hospital and physiotherapists at the hospital and at home currently play an important role in the rehabilitation process for TKA patients.

This study involved the same health care professionals and the same treatment strategies that are currently in use in the Netherlands, but one patient group additionally received CPM at home.

The expected effect of CPM treatment was a quicker restoration of RoM, resulting in improved ADL function during the first three months after surgery. Knee flexion values of $95^{\circ}$ and $105^{\circ}$ are regarded as RoM benchmarks 27 in the functional recovery after CPM. While $95^{\circ}$ of knee flexion allows normal ADL function, $105^{\circ}$ of flexion provides the opportunity to ride a bicycle. This is of great advantage both in daily life, at least in the Netherlands, and in the rehabilitation from TKA surgery, because cycling allows patients to move the knee much more. We expected that prolonged use of CPM at home would allow patients to achieve these RoM benchmarks earlier in their recovery process.

The study was conducted among patients with limited RoM at the time of hospital discharge. We chose to include this specific subgroup because we believed that CPM might provide the greatest RoM gain in patients with RoM limitations. Furthermore, several authors have stated that patients with poorer function immediately after surgery may well need more attention 2829 . About $50 \%$ of the patients undergoing one of the 160 TKAs performed annually at the Maastricht University Hospital have less than $80^{\circ}$ of RoM four days after surgery and therefore potentially meet the inclusion criteria of the proposed study.

\section{Objective}

Continuous passive motion (CPM) has proved to increase the amount of knee flexion for knee patients in the acute hospital setting (5-10 days). The primary purpose of this randomised controlled trial was to establish whether there is additional longer-term benefit of continuing CPM after hospital discharge.

\section{Research question}

What is the effect on range of motion and functional status of prolonged use of a continuous passive motion device at home in addition to PT, compared to PT alone, in patients with limited flexion range of motion (less than $80^{\circ}$ ) of the knee at discharge from the hospital after total knee arthroplasty? 


\section{Methods/Design}

\section{Study design}

A randomised controlled trial, with blinded treatment allocation, assessment and analysis, was carried out, with local medical ethics committee approval, to assess the added value of prolonged CPM use at home, using function and mobility as the main outcomes.

\section{Participants}

Patients scheduled for unilateral primary TKA between April 1st 2005 and June 30th 2006 in the 'Arthrose kliniek Maastricht' (Maastricht osteoarthritis clinic) programme at the Maastricht University Hospital, the Netherlands, were invited to participate in the study. Subjects were considered eligible if they had less than $80^{\circ}$ of RoM 4 days after surgery, were able to understand and speak Dutch, were not suffering from mental disabilities and were resident within the 'Maastricht Heuvelland' region. Patients were excluded if they needed to stay in hospital for more than five days after surgery or showed relevant co-morbidity influencing mobility (e.g. claudicatio, other prosthesis) or were operated upon by minimally invasive surgery. Patients older than 80 years were also excluded. Eligible patients were contacted one week before the planned surgery, and were randomised into two groups after signing an informed consent form.

\section{Randomisation}

Blocked and concealed randomisation with a block size of four ensured equal distribution of patients over the two treatment groups. Groups were prestratified on preoperative flexion mobility of the knee.

\section{Interventions}

During the in-hospital period, all patients received a standardised PT programme, involving 20 minutes of PT and four hours of CPM use daily for four days. CPM was already applied in the recovery room. Nurses installed the CPM device following standardised procedures. CPM was used for two consecutive hours, twice daily. One session was performed in the evening in order to avoid interfering with other daytime medical and rehabilitation activities. After 5 minutes of warm-up, RoM was set as tolerated by the patients. A description of the treatment protocol after total knee surgery is available on the website of the PT department of the Maastricht University Hospital ${ }^{30}$.

At the end of the in-hospital period, all patients were randomly assigned to one of the following groups: a control group which received semistandardised regular PT and an experimental group which received the same PT intervention in combination with two extra weeks of CPM treatment for four hours daily. CPM was administered in the same fashion as during hospital stay. Patients were able to increase RoM by 
themselves. They kept a patient diary in which they wrote the daily RoM increments as well as the content and duration of their PT sessions, medication use and pain perceived during the day and while on the CPM machine.

From day 18 onward, all patients received regular PT treatment until patients and therapists were satisfied with their overall functioning. The post-clinical PT was standardised in terms of treatment objectives. All patients received treatment consisting of active and passive mobilisation of the knee joint, active strengthening of the m. quadriceps, and training of ADL functions (gait, sit to stand and stair climbing).

\section{Outcome assessment}

After collection of baseline variables before surgery and at hospital discharge, outcome measures were assessed at 17 days, six weeks and three months after surgery, during normal routine assessments at the orthopaedic clinic (table 1) or by visiting the patients at home. The outcome assessor was blinded for the treatment procedure.

Primary outcome measures were:

1. Functional status, using the WOMAC function score 3132 and the Knee Society Score ${ }^{33}$; and

2. Range of motion, assessed with a long-arm goniometer ${ }^{34}$. Secondary outcome measures were:

A. perceived effect, using a seven-point Likert scale;

B. postoperative medication use (amount; type being standardised);

C. satisfaction with treatment, on an 11-point Likert scale;

D. satisfaction with treatment result, on an 11-point Likert scale;

E. adherence to treatment protocols and use of CPM (in hours);

F. quantity, duration and nature of PT intervention.

The study design is depicted in figure 1

The first primary endpoint of the study was on day 17 after surgery, which was the time when the experimental treatment stopped and short-term effects were measured.

The second endpoint was at three months after surgery. 
Fig 1: study design and outcome assessment.

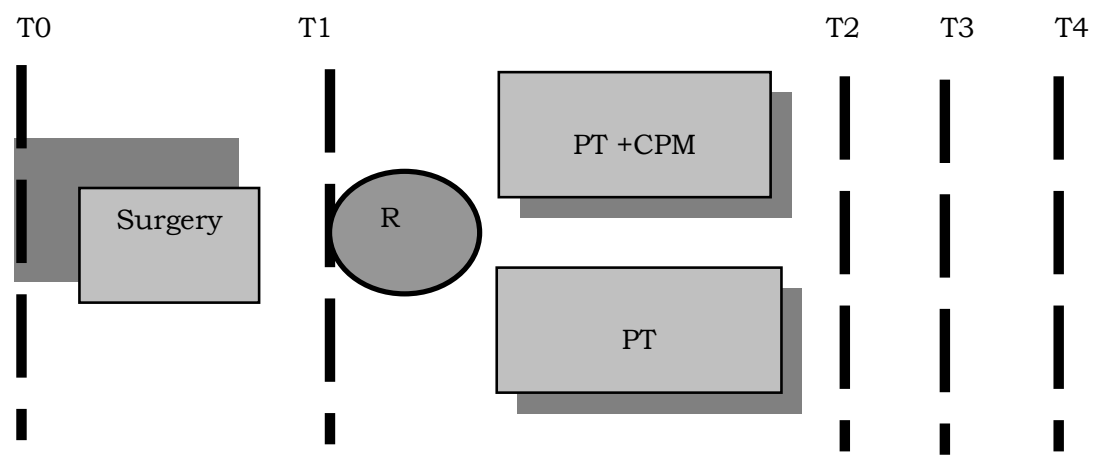

TO baseline assessment, one week before surgery,

T1 assessment 4 days after surgery,

T2 assessment 17 days after surgery,

T3 assessment 6 weeks after surgery

T3 assessment 3 months after surgery

$\mathrm{R}=$ randomisation

Table 1 shows the timing of the outcome assessment

Table 1: Timing of the outcome assessment

\begin{tabular}{|c|c|c|c|c|c|}
\hline & $\begin{array}{l}\text { T0 } \\
1 \text { week } \\
\text { prior to } \\
\text { surgery }\end{array}$ & $\begin{array}{l}\text { T1 } \\
\text { End of } \\
\text { clinical } \\
\text { phase }\end{array}$ & $\begin{array}{l}\text { T2 } \\
17 \text { days } \\
\text { after sur- } \\
\text { gery }\end{array}$ & $\begin{array}{l}\text { T3 } \\
6 \text { weeks } \\
\text { after sur- } \\
\text { gery }\end{array}$ & $\begin{array}{l}\text { T4 } \\
12 \text { weeks } \\
\text { after sur- } \\
\text { gery }\end{array}$ \\
\hline Range of motion & $\mathrm{X}$ & $\mathrm{X}$ & $\mathrm{X}$ & $\mathrm{X}$ & $\mathrm{X}$ \\
\hline Knee Society Score & $\mathrm{X}$ & $\mathrm{X}$ & $\mathrm{X}$ & $\mathrm{X}$ & $\mathrm{X}$ \\
\hline WOMAC & $\mathrm{X}$ & & $\mathrm{X}$ & $\mathrm{X}$ & $\mathrm{X}$ \\
\hline Perceived effect & & & $\mathrm{X}$ & $\mathrm{X}$ & $\mathrm{X}$ \\
\hline Pain medication & & $\mathrm{X}$ & $\mathrm{X}$ & $\mathrm{X}$ & $\mathrm{X}$ \\
\hline $\begin{array}{l}\text { Satisfaction with treat- } \\
\text { ment }\end{array}$ & & & $\mathrm{X}$ & $\mathrm{X}$ & $\mathrm{x}$ \\
\hline Satisfaction with results & & & $\mathrm{X}$ & $\mathrm{X}$ & $\mathrm{X}$ \\
\hline Adherence & & & $\mathrm{X}$ & & \\
\hline $\begin{array}{l}\text { Quantity, duration and } \\
\text { kind of treatment }\end{array}$ & & & $\mathrm{X}$ & $\mathrm{X}$ & $\mathrm{X}$ \\
\hline
\end{tabular}

RoM was measured actively as well as passively using a large goniometer following the method described by Brosseau ${ }^{34}$. The intraobserver reliability of this method for knee flexion is 0.99 , that for active extension 0.97 , and the criterion validity for knee flexion is 0.98 , that for extension $0.42^{34}$.

Functional status was measured using two scales, the joint specific Knee Society Scale (KSS) ${ }^{33}$ and the disease-specific Western Ontario and McMaster University Osteoarthritis index (WOMAC) ${ }^{31}$.

The KSS is concise and easy to use. It represents a clear attempt to separate knee function from overall patient functional status. 
Bach et $\mathrm{al}^{35}$ reported that the reproducibility of the knee score is poor, whilst the function score shows good reproducibility. The construct validity of the KSS is good ${ }^{36}$.

The WOMAC Osteoarthritis Index is a disease-specific questionnaire developed specifically for people with osteoarthritis of the hip and knee. It is a self-administered, 3-dimension, 24-item instrument. The three dimensions of the WOMAC are pain, stiffness, and physical function. Scoring of the WOMAC ranges from 'none' to 'extreme'. Scale sum scores have been standardised (0-100), with high values indicating less pain or better physical functioning ${ }^{32}$. The WOMAC questionnaire is generally acknowledged to have good validity, reliability and responsiveness. We used the Dutch version of WOMAC ${ }^{32}$.

At follow-up, patients were asked to judge the effect of the surgery on a seven-point Likert scale ranging from 'worse than ever' status to 'completely recovered'37.

Postoperative medication use and adherence to the treatment protocols were measured using a patient diary.

The primary effect measurement was scheduled for the 17th day after surgery, while follow-up measurements were scheduled at six weeks and three months after surgery.

\section{Power analysis}

The number of subjects required to achieve statistical significance was determined by means of a power analysis. We assumed that a difference of more than $5^{\circ}$ of knee flexion mobility (SD $8^{\circ}$ ) at the end of the CPM application would be clinically relevant. With an alpha of 0.05 , and a power of $80 \%$, we needed 28 patients per group to prove this.

\section{Data analysis}

Data was stored and analysed with SPSS-12.0 After checks for missing values and normality, linear regression techniques were applied by a blinded analyst using the 'intention-to-treat' principle. Primary and secondary outcome measures were reported for the in-hospital and home situations, and for six-week and three-month follow-up. Means and $95 \%$ confidence intervals were calculated.

The primary research question was tested using Student's t-tests with a p-value of 0.05 being regarded as statistically significant.

\section{Results}

Of the 147 patients who were scheduled for surgery between April $1^{\text {st }}$ 2005 and June 30th 2006, 60 were included in the study. Thirty-five patients were not considered eligible, for the following reasons. Five patients were not residents of the Maastricht region, 10 patients had relevant co-morbidity or had RA as an underlying problem and 20 patients were older than 80 years. 
Figure 2: Flow chart of subjects through the trial.

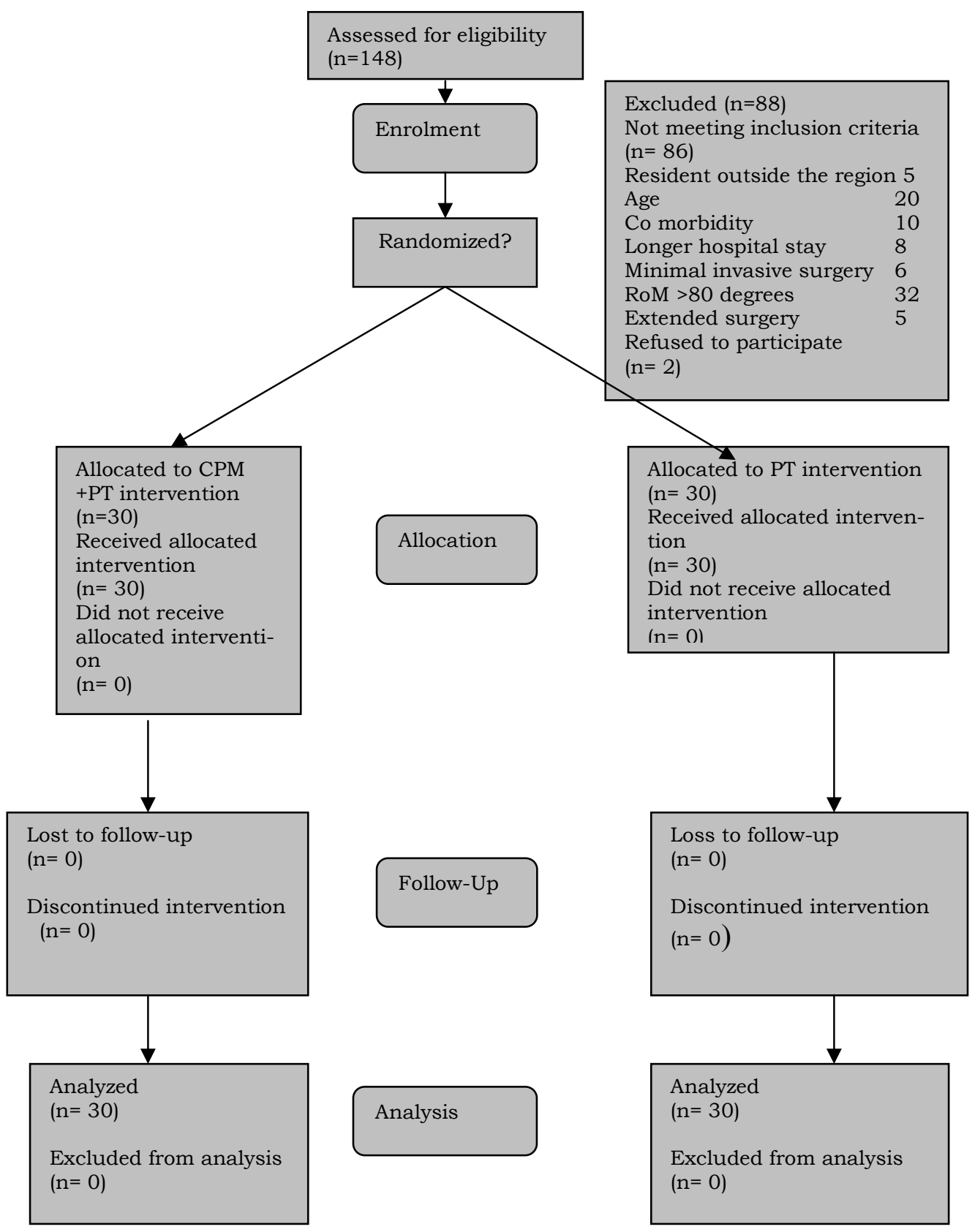


Another 52 patients were excluded, for the following reasons: 32 were excluded because their active knee RoM was over $80^{\circ}$ at T1; eight patients needed to stay in hospital for more than five days after surgery and six were operated upon using minimally invasive surgery. In four cases, surgery was more extensive. Two patients refused to participate because of bad experiences with CPM treatment during the in-hospital period.

The baseline characteristics of the patients in the two groups were similar in terms of clinical and demographic characteristics. Although we prestratified on preoperative active RoM (strata $>100^{\circ}<$ ), mean preoperative flexion RoM was slightly higher in the CPM group. At T1, RoM was similar in both groups. Both groups also showed comparable levels of functional ability (tables 2 and 3).

Table 2: Subject characteristics and preoperative assessments of RoM and functional status

\begin{tabular}{|c|c|c|c|c|}
\hline TO & $\mathrm{PT}+\mathrm{CPM}$ & $\mathrm{n}=30$ & $\mathrm{Pt}$ & $=30$ \\
\hline \multirow[t]{2}{*}{ Gender $\%$ female } & 60 & & 70 & \\
\hline & Mean & sd & Mean & sd \\
\hline Age & 64.1 & 8.1 & 65 & 9.1 \\
\hline $\begin{array}{l}\text { Active RoM before surgery } \\
\text { (flexion to extension) }\end{array}$ & 104.9 & 13.8 & 100.7 & 13.2 \\
\hline Passive RoM before surgery & 110.2 & 14.0 & 105.9 & 13.8 \\
\hline Active extension & 4.8 & 4.6 & 6.2 & 5.0 \\
\hline Active flexion & 109.7 & 12.1 & 106.9 & 12.3 \\
\hline Passive extension & 3.3 & 4.3 & 4.7 & 4.3 \\
\hline Passive flexion & 113.5 & 12.4 & 110.6 & 11.8 \\
\hline KSS knee & 53.5 & 16.4 & 47.5 & 15.3 \\
\hline KSS function & 61.5 & 10.6 & 52.3 & 18.7 \\
\hline WOMAC score & 54.8 & 17.7 & 51.2 & 13.6 \\
\hline Pain subscale & 10.6 & 4.7 & 10.5 & 3.1 \\
\hline Stiffness subscale & 4. 0 & 1.9 & 3.8 & 2.1 \\
\hline Difficulty subscale & 40.2 & 13.2 & 36.9 & 11.7 \\
\hline
\end{tabular}

Table 3: Baseline measurement (T1) of RoM and KSS

\begin{tabular}{|c|c|c|c|c|}
\hline T1 & $\mathrm{PT}+\mathrm{CPM}$ & $\mathrm{n}=30$ & Pt & $\mathrm{n}=30$ \\
\hline Active RoM at T1 & 66.1 & 8.9 & 66.6 & 6.8 \\
\hline Passive RoM at $\mathrm{T} 1$ & 71.1 & 8.4 & 72.5 & 5.7 \\
\hline Active extension & 8.9 & 4.4 & 8.1 & 4.4 \\
\hline Active flexion & 75 & 6.9 & 74.7 & 4.5 \\
\hline Passive extension & 6.7 & 4.1 & 6.1 & 4.2 \\
\hline Passive flexion & 77.8 & 7.1 & 78.6 & 3.8 \\
\hline Knee society knee score at T1 & 55.9 & 17 & 50.0 & 17.6 \\
\hline Knee society function score at $\mathrm{T} 1$ & 25.8 & 14 & 26 & 13.5 \\
\hline
\end{tabular}

$\mathrm{T} 1=$ day of discharge from the hospital, i.e. day 4 after surgery 


\section{Primary outcome measures}

Range of motion

A trend in favour of prolonged CPM use was found at the end of the treatment period. In terms of total RoM, the CPM group achieved $5^{\circ}$ more RoM than the PT group (Table 4). RoM in the CPM group improved $6^{\circ}$ more during the active treatment period $\left(17.7^{\circ}\right.$ over $\left.11.6^{\circ}\right)$.

At follow-up, these differences had faded. At six weeks and three months, we could not detect any difference in RoM between the two treatment strategies (figure 3). In terms of the number of patients achieving the flexion RoM benchmarks of $95^{\circ}$ and $105^{\circ}$, no differences were found between the two groups at any of the outcome measurements (table 5).

Table 4: Primary outcome measurements on day 17

\begin{tabular}{|c|c|c|c|c|c|c|}
\hline T2 ROM & $\begin{array}{l}\text { PT + CPM } \\
\text { Mean }\end{array}$ & $\begin{array}{l}n=30 \\
\text { sd }\end{array}$ & $\begin{array}{l}\text { Pt } \\
\text { Mean }\end{array}$ & $\begin{array}{r}n=30 \\
\text { sd }\end{array}$ & $95 \%$ & $\mathrm{CI}$ \\
\hline Active RoM at T2 & 83.6 & 11.4 & 78.6 & 8.7 & -0.2 & -10.3 \\
\hline Passive RoM at T2 & 88.7 & 10.4 & 84.0 & 9.9 & -0.5 & -10.0 \\
\hline Delta active RoM T1-T2 & 17.5 & 13.4 & 11.9 & 9.8 & -0.5 & -11.6 \\
\hline Delta passive RoM T1-T2 & 17.6 & 12.9 & 11.6 & 9.4 & 0.02 & -11.9 \\
\hline Extension active & 6.3 & 3.9 & 8.1 & 4.8 & -4.1 & $-\quad 0.4$ \\
\hline Extension passive & 4.3 & 3.1 & 5.7 & 4.6 & -3.4 & 0.6 \\
\hline Flexion active & 89.9 & 9.1 & 86.7 & 8.5 & -1.4 & 7.8 \\
\hline Flexion passive & 93 & 8.8 & 89.7 & 9.6 & -1.4 & 8.1 \\
\hline KSS knee score & 67.6 & 19.6 & 67.3 & 14.9 & -8.7 & $-\quad 9.3$ \\
\hline KSS function score & 43 & 14.6 & 39.8 & 21.1 & -4.7 & -11.3 \\
\hline WOMAC score & 69.9 & 15.9 & 65.4 & 16.4 & -3.9 & -12.9 \\
\hline WOMAC pain & 15.8 & 4.7 & 15.3 & 4.1 & -2.9 & $\begin{array}{l}-\quad 1.7 \\
\end{array}$ \\
\hline WOMAC stiffness & 5.0 & 1.8 & 4.8 & 1.6 & -0.7 & 1.1 \\
\hline WOMAC difficulty & 49.1 & 11.9 & 45.3 & 12.3 & -2.5 & -10.1 \\
\hline
\end{tabular}

$\mathrm{T} 1=$ day of discharge from the hospital, i.e. day 4 after surgery

$\mathrm{T} 2=17$ days after surgery

Table 5: Number of patients reaching flexion benchmarks at T2 and T3

\begin{tabular}{|c|c|c|c|c|c|c|c|c|}
\hline & \multicolumn{4}{|c|}{ T2 17 days after surgery } & \multicolumn{4}{|c|}{ T3 6 weeks after surgery } \\
\hline Group & $<$ & 0 & $<$ & $>$ & & $>$ & $<$ & $>$ \\
\hline $\mathrm{CPM}+\mathrm{PT}$ & 25 & 5 & 27 & 3 & 10 & 20 & 20 & 1 \\
\hline PT & 24 & 6 & 28 & 2 & 10 & 20 & 22 & 8 \\
\hline
\end{tabular}

\section{Functional status}

Although the experimental group scored slightly better on the KSS functional status score and the WOMAC functional difficulty score, no significant differences were found between the two groups, neither at day 17 nor at either of the follow-up measurements (tables 4 and 6). 


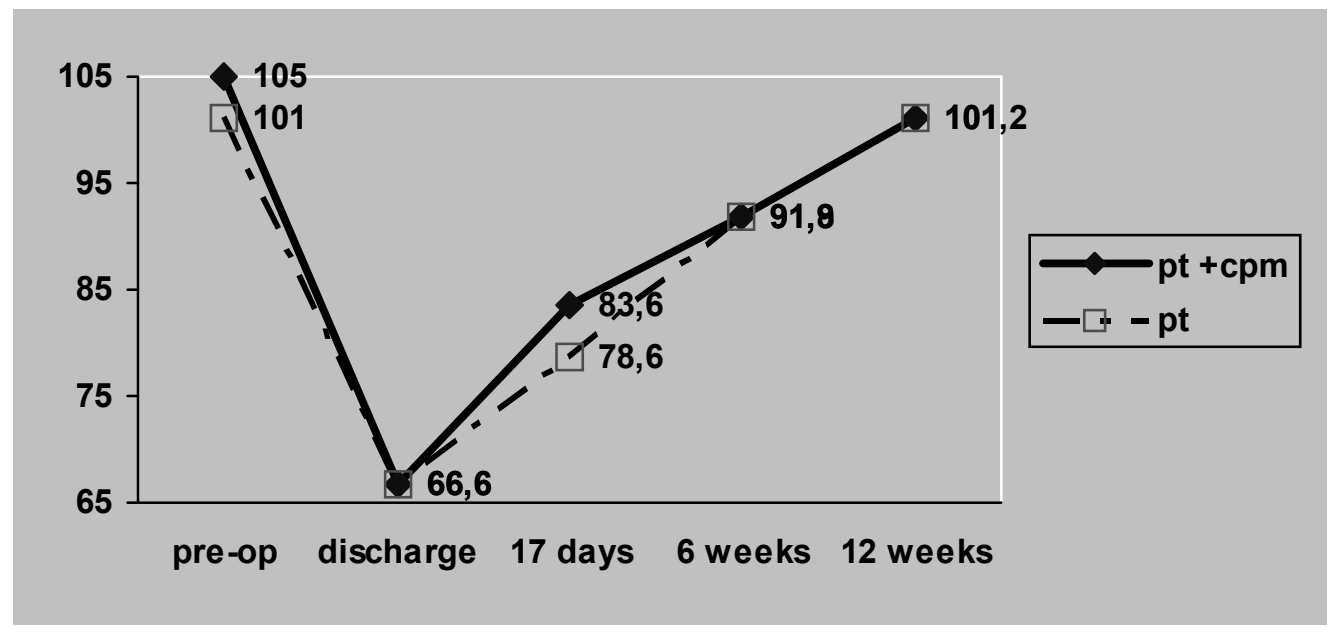

\section{Secondary outcome}

No significant differences were found on any of the secondary outcome measures at any single outcome measurement. Treatment content was similar in both groups, as were the number of treatment sessions and the time spent on individual sessions. As early as day 17, the majority of the patients scored their status (perceived effect) as 'better' compared to the situation before surgery. Patients were generally satisfied with their individual treatment and with treatment results (table 7).

Adherence to the CPM intervention was very high: 29 of the 30 patients followed the prescribed regimen. One patient reduced CPM use to two hours from day 10 onwards, because her knee RoM was over $105^{\circ}$, and she felt uncomfortable with four hours of CPM use when reaching high degrees of flexion.

\section{Complications}

One subject in each group had to undergo knee manipulation under anaesthesia between six weeks and three months after surgery.

Secondary analysis on the subgroup of the patients having less than $68^{\circ}$ (0-50 percentile) of active RoM at discharge showed that these patients benefited more from CPM treatment. They improved $11^{\circ}$ more $\left(22^{\circ}\right.$ versus $11^{\circ}$ in the PT group) during the active treatment fortnight. However, these differences in range of motion benefit had also faded at six-week follow-up. 


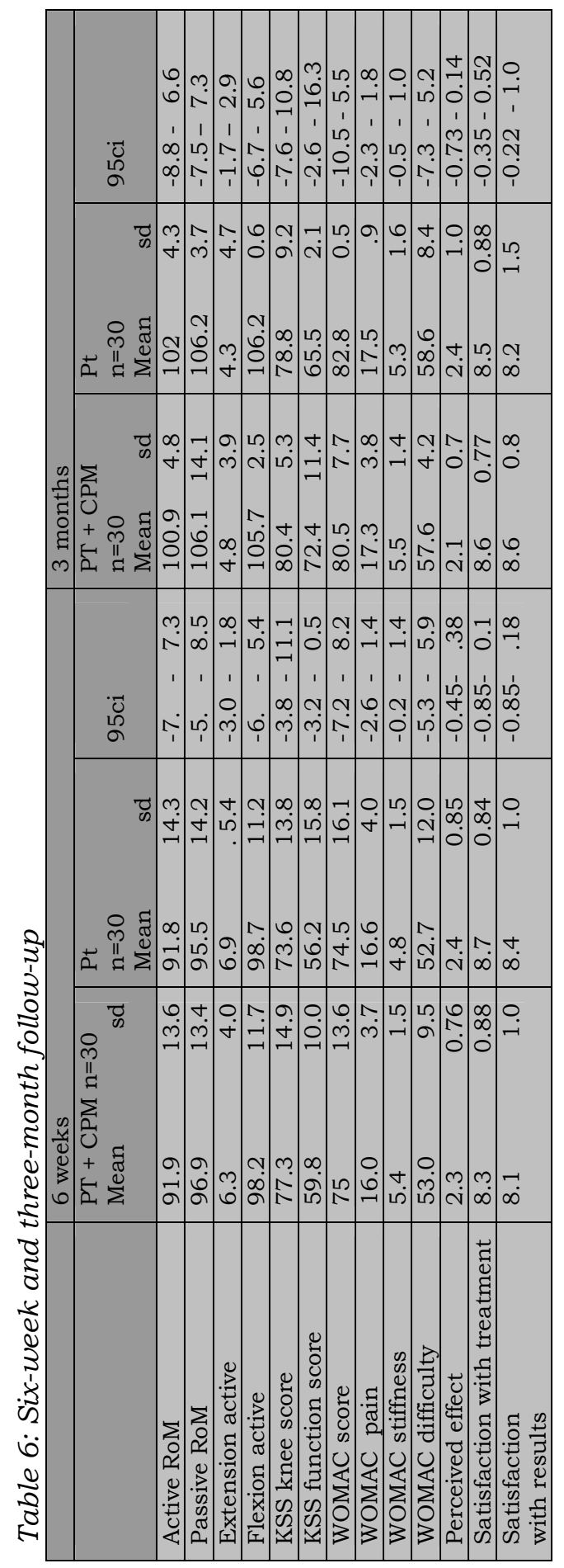


Table 7: Secondary outcome assessment on day 17

\begin{tabular}{|c|c|c|c|c|c|c|}
\hline \multirow[t]{2}{*}{ T2 ROM } & \multicolumn{2}{|c|}{$\mathrm{PT}+\mathrm{CPM}$} & & \multicolumn{2}{|c|}{$95 \% \mathrm{CI}$} \\
\hline & Mean & sd & Mean & sd & & \\
\hline Number of treatment sessions & 4.8 & 1.5 & 5.1 & 1.4 & -1.05 & 0.44 \\
\hline Duration per session & 29.5 & 5.5 & 28.4 & 5.9 & -2.0 & 4.13 \\
\hline Perceived effect & 3.2 & 1.5 & 3.0 & 1.5 & -0.5 & 1.0 \\
\hline Satisfaction with treatment & 8.2 & 0.8 & 8.5 & 0.7 & -1.0 & -0.1 \\
\hline Satisfaction with results & 7.4 & 1.4 & 7.8 & 1.5 & -1.2 & -0.3 \\
\hline Adherence & $97 \%$ & & $100 \%$ & & & \\
\hline
\end{tabular}

\section{Discussion}

The present study demonstrated improvement in total RoM at the end of the prolonged period of CPM use. However, this did not translate into any functional benefits. Our findings thus suggest that, although CPM produces benefits in knee RoM in the short term, it does not result in additional RoM in the longer term, or in any functional gain. Since we did not find any difference in the numbers of patients achieving the clinically important benchmarks of $95^{\circ}$ and $105^{\circ}$, neither at the end of the treatment period nor at follow-up, it is doubtful whether the additional degrees of RoM are of clinical importance.

Although our study population was a selection of patients with limited RoM at discharge, our results confirm those presented in systematic reviews 2425 , implying that patients with limited RoM exhibit comparable improvements to the basic population of patients after TKA. Our hypothesis that this group might benefit more from the CPM application was not supported. Our long-term results were comparable to those reported by others 121538 . Kumar et al 10 and Leach et al 23 reported greater RoM at six-week follow-up. A possible explanation might be that they included patients, regardless of RoM and therefore found somewhat better RoM.

Like previous researchers 9151639 , we did not detect any differences in functional status between the groups. The addition of CPM did not seem to lead to measurable functional benefits. Denis et al. ${ }^{16}$ surmised that subjects who received additional CPM could even have poorer functional abilities, because they remained inactive during the CPM interventions. We did not detect a decrease in functional activities in the CPM group. A large proportion of all subjects, regardless of research group allocation, reported functional gains on all outcome measurements. Patients with limited RoM in the early stages of recovery seemed to consistently improve over time.

We chose total RoM as the outcome instead of focusing on flexion RoM because several authors 8 40-42 have already reported on adverse effects of CPM application on extension range. Although extension RoM in our 
patients was limited in the short term after TKA, we did not detect any difference between the two groups. In fact, we found slightly better extension RoM in the CPM group. Our extension deficits were comparable to those already reported by others 9101622 .

We found small effects on range of motion at the end of the active treatment period, which faded during four weeks of follow-up in which the patients received regular PT treatment. This suggests that although adding 14 days of CPM is beneficial for short-term RoM, the improvement does not last. An alternative hypothesis might be that CPM treatment should be maintained for an even longer period for effects to take root. Our study results do not rule out this hypothesis.

\section{Study limitations}

Milne et al. ${ }^{24}$ suggested in their review that future research should focus on the effects of different characteristics of application, such as total duration of treatment and intensity of CPM interventions, and on effects in different populations. We chose to include patients with limited range of motion at the end of the in-hospital period, postulating that these patients might benefit most from extra CPM treatment. Perhaps our choice of target population was wrong. In any case, one should keep in mind that our conclusions are limited to comparable populations, post-clinical PT treatment and CPM application protocols similar to those described in our clinical trial. This may be obvious, but as several authors 4344 have already pointed out, the treatment approaches used after TKA vary greatly among rehabilitation providers. The external validity of our study may therefore be limited if protocols differ greatly.

We protocolised the post-clinical PT in terms of treatment objectives, leaving individual therapists some leeway to specify these objectives at their own discretion. This may have led to differences in treatment content between individual therapists, which might have affected the contrast between the two interventions. However, we did not detect any major differences in PT treatment from the patient diaries in which therapists wrote down the content of the individual treatment sessions during the active CPM treatment period.

CPM treatment in the home situation was a self-management treatment. Patients received the CPM machine, which was adjusted to their individual needs and characteristics. Patients were instructed how to use it and for how long. During the next fortnight, patients managed treatment on their own or with the help of their spouses. Although they were instructed to call the researcher if they had any problems with the machine, this only occurred twice. Although analysis of the patient diaries in which they wrote daily CPM increments and usage time did not indicate any misuse in CPM application, and although self-management is usual care for at-home CPM use in the Netherlands, it may have led to sub-maximal use of the CPM device. 
Our study has many characteristics that contribute to the validity of the results, such as comparability at baseline, adherence to interventions and the use of one outcome assessor to minimise noise due to problems of interobserver reproducibility. The most important favourable feature of our study was the absolute lack of drop-outs and loss to follow-up. We arranged the trial in such a fashion that all measurements were taken either during regular follow-up visits to the hospital or by visiting patients at home. Although this was extremely time-consuming and we were lucky that not a single patient had significant other problems during our follow-up period, this led to a zero percent drop-out and loss to follow-up rate.

\section{Conclusions}

Although our results indicate that prolonged CPM use has a short-term effect on RoM, standard implementation of prolonged CPM use in patients with limited RoM at hospital discharge should be reconsidered, since neither long-term effects nor transfer to better functional performance was detected.

\section{Competing interests}

The authors declare that they have no competing interests.

\section{Authors' contributions}

AFL participated in the design of the study and participated in the assessments and follow-ups, statistical analysis and writing. YC and EW participated in design and in the assessments and follow-ups. MvS, RG, PvdB and RdB participated in the design. All authors have read and approved the final manuscript. 


\section{References}

1. Peat G, McCarney R, Croft P. Knee pain and osteoarthritis in older adults: a review of community Burden and current use of primary health care. Ann Rheum Dis 2001;60:91-97.

2. Jordan KM, Arden NK, Doherty M, Bannwarth B, Bijlsma JW, Dieppe P, et al. EULAR Recommendations 2003: an evidence based approach to the management of knee osteoarthritis: Report of a Task Force of the Standing Committee for International Clinical Studies Including Therapeutic Trials (ESCISIT). Ann Rheum Dis 2003;62(12):1145-55.

3. Salter RB, Simmonds DF, Malcolm BW, Rumble EJ, MacMichael D, Clements ND. The biological effect of continuous passive motion on the healing of full-thickness defects in articular cartilage. An experimental investigation in the rabbit. J Bone Joint Surg Am 1980;62(8):1232-51.

4. Salter RB, Hamilton HW, Wedge JH, Tile M, Torode IP, O'Driscoll SW, et al. Clinical application of basic research on continuous passive motion for disorders and injuries of synovial joints: a preliminary report of a feasibility study. J Orthop Res 1984;1(3):325-42.

5. Salter RB. The biologic concept of continuous passive motion of synovial joints. The first 18 years of basic research and its clinical application. Clin Orthop Relat Res 1989(242):12-25.

6. Salter RB. The physiologic basis of continuous passive motion for articular cartilage healing and regeneration. Hand Clin 1994;10(2):2119.

7. Coutts RD, Toth C, Kaita JH. The role of continuous passive motion in the rehabilitation of the total knee patient In: DS H, editor. TKA: $a$ comprehensive approach. Baltimore: Williams \& Wilkins, 1984:126-132.

8. Pope RO, Corcoran S, McCaul K, Howie DW. Continuous passive motion after primary total knee arthroplasty. Does it offer any benefits? $J$ Bone Joint Surg Br 1997;79(6):914-7.

9. McInnes J, Larson MG, Daltroy LH, Brown T, Fossel AH, Eaton HM, et al. A controlled evaluation of continuous passive motion in patients undergoing total knee arthroplasty. Jama 1992;268(11):1423-8.

10. Kumar PJ, McPherson EJ, Dorr LD, Wan Z, Baldwin K. Rehabilitation after total knee arthroplasty: a comparison of 2 rehabilitation techniques. Clin Orthop Relat Res 1996(331):93-101.

11. Lenssen AF, Bie de RA, Bulstra SK, Steijn van MJA. Continuous passive motion in rehabilitation following total knee arthroplasty: a Randomised controlled trial. Physical Therapy Reviews 2003;8(3):123129.

12. Johnson DP, Eastwood DM. Beneficial effects of continuous passive motion after total condylar knee arthroplasty. Ann $R$ Coll Surg Engl 1992;74(6):412-6.

13. Vince KG, Kelly MA, Beck J, Insall JN. Continuous passive motion after total knee arthroplasty. J Arthroplasty 1987;2(4):281-4.

14. Montgomery F, Eliasson M. Continuous passive motion compared to active physical therapy after knee arthroplasty: similar hospitalization times in a randomized study of 68 patients. Acta Orthop Scand 1996;67(1):7-9.

15. Bennett LA, Brearley SC, Hart JA, Bailey MJ. A comparison of 2 continuous passive motion protocols after total knee arthroplasty: a controlled and randomized study. J Arthroplasty 2005;20(2):225-33. 
16. Denis M, Moffet H, Caron F, Ouellet D, Paquet J, Nolet L. Effectiveness of continuous passive motion and conventional physical therapy after total knee arthroplasty: a randomized clinical trial. Phys Ther 2006;86(2):174-85.

17. Walker RH, Morris BA, Angulo DL, Schneider J, Colwell CW, Jr. Postoperative use of continuous passive motion, transcutaneous electrical nerve stimulation, and continuous cooling pad following total knee arthroplasty. J Arthroplasty 1991;6(2):151-6.

18. Nielsen PT, Rechnagel K, Nielsen SE. No effect of continuous passive motion after arthroplasty of the knee. Acta Orthop Scand 1988;59(5):580-1.

19. Beaupre LA, Davies DM, Jones CA, Cinats JG. Exercise combined with continuous passive motion or slider board therapy compared with exercise only: a randomized controlled trial of patients following total knee arthroplasty. Phys Ther 2001;81(4):1029-37.

20. Worland RL, Arredondo J, Angles F, Lopez-Jimenez F, Jessup DE. Home continuous passive motion machine versus professional physical therapy following total knee replacement. $J$ Arthroplasty 1998;13(7):784-7.

21. Chen B, Zimmerman JR, Soulen L, DeLisa JA. Continuous passive motion after total knee arthroplasty: a prospective study. Am J Phys Med Rehabil 2000;79(5):421-6.

22. Chiarello CM, Gundersen L, O'Halloran T. The effect of continuous passive motion duration and increment on range of motion in total knee arthroplasty patients. J Orthop Sports Phys Ther 1997;25(2):119-27.

23. Leach W, Reid J, Murphy F. Continuous passive motion following total knee replacement: a prospective randomized trial with follow-up to 1 year. Knee Surg Sports Traumatol Arthrosc 2006.

24. Milne S, Brosseau L, Robinson V, Noel MJ, Davis J, Drouin H, et al. Continuous passive motion following total knee arthroplasty. Cochrane Database Syst Rev 2003(2):CD004260.

25. Lenssen AF, Koke AJA, Bie de RA, Geesink RGT. Continuous passive motion in rehabilitation following total knee arthroplasty; short and long term effects on range of motion. Physical Therapy reviews 2003;8(3):113-121.

26. Lenssen AF, Köke AJA, De Bie RA. Effectiviteit van continuous passive motion in de klinische nabehandeling van patienten na een totale knie prothese: een systematische review. Nederlands Tijdschrift voor Fysiotherapie 2001; dec 2001(6):152-158.

27. Miner AL, Lingard EA, Wright EA, Sledge CB, Katz JN. Knee range of motion after total knee arthroplasty: how important is this as an outcome measure? J Arthroplasty 2003;18(3):286-94.

28. Jones CA, Voaklander DC, Suarez-Alma ME. Determinants of function after total knee arthroplasty. Phys Ther 2003;83(8):696-706.

29. Ritter MA, Berend ME, Harty LD, Davis KE, Meding JB, Keating EM. Predicting range of motion after revision total knee arthroplasty: clustering and log-linear regression analyses. $J$ Arthroplasty 2004;19(3):338-43.

30. LenssenAF

http://www.azm.nl/professionals/overige+afdelingen/overzicht+overige +afdelingen/fysiotherapie/onderzoek/treatmentprotocoltkpshortenglish .pdf., 2006. 
31. Bellamy N, Buchanan WW, Goldsmith $\mathrm{CH}$, Campbell J, Stitt LW. Validation study of WOMAC: a health status instrument for measuring clinically important patient relevant outcomes to antirheumatic drug therapy in patients with osteoarthritis of the hip or knee. $J$ Rheumatol 1988;15(12):1833-40.

32. Roorda LD, Jones CA, Waltz M, Lankhorst GJ, Bouter LM, van der Eijken JW, et al. Satisfactory cross cultural equivalence of the Dutch WOMAC in patients with hip osteoarthritis waiting for arthroplasty. Ann Rheum Dis 2004;63(1):36-42.

33. Insall JN, Dorr LD, Scott RD, Scott WN. Rationale of the Knee Society clinical rating system. Clin Orthop Relat Res 1989(248):13-4.

34. Brosseau L, Balmer S, Tousignant M, O'Sullivan JP, Goudreault C, Goudreault $\mathrm{M}$, et al. Intra- and intertester reliability and criterion validity of the parallelogram and universal goniometers for measuring maximum active knee flexion and extension of patients with knee restrictions. Arch Phys Med Rehabil 2001;82(3):396-402.

35. Bach CM, Nogler M, Steingruber IE, Ogon M, Wimmer C, Gobel G, et al. Scoring systems in total knee arthroplasty. Clin Orthop Relat Res 2002(399):184-96.

36. Lingard EA, Katz JN, Wright RJ, Wright EA, Sledge CB. Validity and responsiveness of the Knee Society Clinical Rating System in comparison with the SF-36 and WOMAC. J Bone Joint Surg Am 2001;83-A(12):1856-64.

37. Guyatt GH, Townsend M, Berman LB, Keller JL. A comparison of Likert and visual analogue scales for measuring change in function. $J$ Chronic Dis 1987;40(12):1129-33.

38. McDonald S, Hetrick S, Green S. Pre-operative education for hip or knee replacement. Cochrane Database Syst Rev 2004(1):CD003526.

39. MacDonald SJ, Bourne RB, Rorabeck CH, McCalden RW, Kramer J, Vaz M. Prospective randomized clinical trial of continuous passive motion after total knee arthroplasty. Clin Orthop Relat Res 2000(380):30-5.

40. Ritter MA, Gandolf VS, Holston KS. Continuous passive motion versus physical therapy in total knee arthroplasty. Clin Orthop Relat Res 1989(244):239-43.

41. Ververeli PA, Sutton DC, Hearn SL, Booth RE, Jr., Hozack WJ, Rothman RR. Continuous passive motion after total knee arthroplasty. Analysis of cost and benefits. Clin Orthop Relat Res 1995(321):208-15.

42. Laskin RS, Beksac B. Stiffness after total knee arthroplasty. $J$ Arthroplasty 2004;19(4 Suppl 1):41-6.

43. Jones DL, Westby MD, Greidanus N, Johanson NA, Krebs DE, Robbins L, et al. Update on hip and knee arthroplasty: current state of evidence. Arthritis Rheum 2005;53(5):772-80.

44. Naylor J, Harmer A, Fransen M, Crosbie J, Innes L. Status of physiotherapy rehabilitation after total knee replacement in Australia. Physiother Res Int 2006;11(1):35-47. 

8

General discussion 
Total knee arthroplasty (TKA) is currently one of the most successful orthopaedic operations ${ }^{1-3}$. This thesis reports on research into various aspects of peri-operative physiotherapeutic care in TKA patients. The main focus of the research was to asses the effectiveness of continuous passive motion (CPM) after surgery. This was done by means of a systematic review (chapter 2), followed by two randomized controlled trials (RCTs) to compare physiotherapy (PT) with a treatment regimen in which CPM was added to PT, in the clinical phase (chapter 3 ) and during the period immediately after hospital discharge (chapters 6 and 7).

Where Chapters 4 and 5 discussed the reproducibility of the main outcome assessment in CPM care and the efficiency of postoperative inhospital PT, This final chapter critically reviews our main findings and conclusions.

\section{Systematic review}

A systematic review is a relatively cheap and quick way to evaluate available evidence. It includes a comprehensive search strategy and an explicit method to appraise and synthesize the available evidence from individual studies ${ }^{4}$. The methodological quality of the studies that were included in our review was assessed by means of the MaastrichtAmsterdam consensus list ${ }^{6}$, using only items reflecting internal validity. As in many other systematic reviews, large differences in reported methodological quality items were found, ranging from two to eight points out of a possible ten.

Problems were twofold. Firstly, authors did not always consider important methodological aspects of their research designs, leading to possible bias. Secondly, estimation of study quality in systematic reviews is based on the published reports, and quality assessment is frequently hampered by a lack of information on several aspects of internal validity in the underlying studies. Our review found that information on cointerventions, compliance and analyses in the underlying RCTs were inadequately reported.

Although the CONSORT statement, which recommends a list of 21 items which should be included in a trial report ${ }^{7} 8$, was introduced in 1996, we did not detect an improvement in methodological quality in the study reports published in later years. Hopefully, further dissemination of the Consort statement will improve the information provided in study reports in the near future.

We concluded that there is moderate but conflicting evidence for the short-term efficacy of CPM added to PT, versus PT alone. This conclusion was derived from a meta-analysis of 5 studies out of the 15 originally included in our review.

Although our review was restricted to studies reporting on range of motion (RoM) after TKA, 10 studies had to be excluded from the metaanalysis, because there were differences between these studies especially with respect to interventions and the contrast between interventions used in the studies. 
In six studies, CPM was either seen as a replacement for physiotherapy or was compared with other RoM enhancing modalities. This suggests that views regarding the use of CPM differ between professionals, and there is no general consensus about the interpretation of the role of CPM in TKA patient care.

Only for the comparison between CPM added to PT versus PT alone were enough studies sufficiently comparable to allow pooling. All other comparisons were only included in one or two studies, which made pooling irrelevant or at least questionable. Consequently, it was impossible to draw firm conclusions based on these comparisons. The value of such comparisons lies primarily in identifying gaps in the available evidence and alternative practical use of CPM in TKA patients. Of the nine studies ${ }^{9-17}$ investigating CPM added to PT versus PT alone, four had to be removed from the analyses. Two ${ }^{17}$ did not report shortterm outcome, and two other studies ${ }^{10} 16$ had to be removed because the reports lacked the necessary data for inclusion in the analysis.

The internal validity scores of the remaining five studies varied from 3 to 6 points out of 10 . Studies with lower scores were not excluded, because no relation could be found between internal validity and reported outcome.

There may, however, still be some questions about the homogeneity of the five studies ultimately included in our meta-analysis. Although in general, the contrast in all these studies was similar (CPM + PT was compared to PT alone, all used RoM as outcome and all included a short-term outcome assessment), they differed in terms of clinical application characteristics, such as the RoM selected for treatment, the treatment duration or the intensity of application. Also, underlying PT treatments were not uniform in all studies.

Finally, treatment protocols change over time. As our review included study reports published between 1987 and 2001, it may be questioned whether the protocols on which the results in the underlying individual studies are based are still comparable to treatment regimens in use nowadays. If protocols differ from current practice, this might affect the generalizability of the reported outcome to current practice.

\section{The RCT's}

A more time-consuming and expensive way to investigate the effectiveness of interventions is to conduct a randomized controlled trial (RCT), since well-conducted RCTs provide the best evidence for the effectiveness of interventions ${ }^{18} 19$.

This thesis contains the reports on three RCTs. the next subsections discuss aspects of the internal validity of all three trials, as well as similarities and differences between the three RCTs. 


\section{Internal validity of studies}

\section{Randomization}

In the studies on the effect of CPM use in the in-hospital phase (chapter 3) and the trial on efficiency of in-hospital PT (chapter 5) we chose cluster randomization ${ }^{20}$ of treatment weeks rather than individual randomization. Clustering participants over treatment weeks ensured that all patients operated upon in one week were receiving the same treatment. We believe that this led to less bias from contamination than would have been introduced by individual randomization, as individual randomization would lead to patients receiving experimental treatment being in the hospital at the same time as control patients. We also believe that contamination would have been more likely to occur if a patient received the extra treatment whilst the patient in the opposite bed did not.

\section{Blinding}

A distinction can be made between blinding of patients, care providers and outcome assessment. In none of the three RCTs were patients or care providers blinded. We believe that blinding patients was simply impossible in these trials. In the trial on the efficiency of in-hospital care it would have been theoretically possible to blind the physiotherapist applying the treatment, if therapists had been divided over two groups and treatment sessions had been given in a closed environment. In our setting, however, this would have a major impact on normal treatment routine. Therefore, we chose to try and blind only the therapists treating patients in the morning sessions. Since the morning sessions were given to both groups, blinding these therapists avoided differences in treatment content and therapists' attitude in the morning sessions. Nevertheless, the participating physical therapists stated that not knowing whether a patient would receive a second treatment session probably changed their attitude somewhat in the morning sessions. Their awareness of achieving clinical goals was raised, because they were unsure whether or not patients would receive a second treatment session. Although they were aware that this attitude might bias study results, their desire to provide optimal patient care overruled the urge to adhere to the treatment protocol.

To minimize any further information bias due to blinding, the outcome assessors and the outcome analyses were blinded. Furthermore, established, valid and reliable measurement tools were used whenever available.

\section{Avoiding co-interventions}

Both of our clinical studies used similar physiotherapeutic cointerventions. Because most other treatments, e.g. pain and fluid management, were protocollized, bias from differences in co-interventions was unlikely. In the follow-up phase, it was more difficult to control for co-interventions. 
In all three trials, outpatient PT was standardized in terms of treatment objectives. All patients received treatment consisting of active and passive mobilization of the knee joint, active strengthening of the m. quadriceps, and training of ADL functions (gait, transferring from sitting to standing and stair climbing). The outpatient physical therapists were not given detailed instructions on treatment intensity. Although our procedure ensured a broad similarity between therapists, individual patients could have received different treatments at different treatment intensities.

The study on post-clinical CPM treatment did check for differences in outpatient treatment content and intensity. Although individual differences were found, no between-group difference in PT administration (selection bias) was detected.

\section{Trial execution}

Designing a trial is a precise and delicate job, in which knowledge of research methodology is an important factor. If a study is well-designed it is crucial that it is also correctly executed. We believe all three studies were carried out properly.

First and foremost, both the efficiency trial and the prolonged use of CPM trial were executed within the planned timeframe. The clinical study was set up as a pilot study and the inclusion period was set at five months. No prior power calculation was performed for this trial.

Figure 1: expected and included patient flow of the prolonged CPM study.

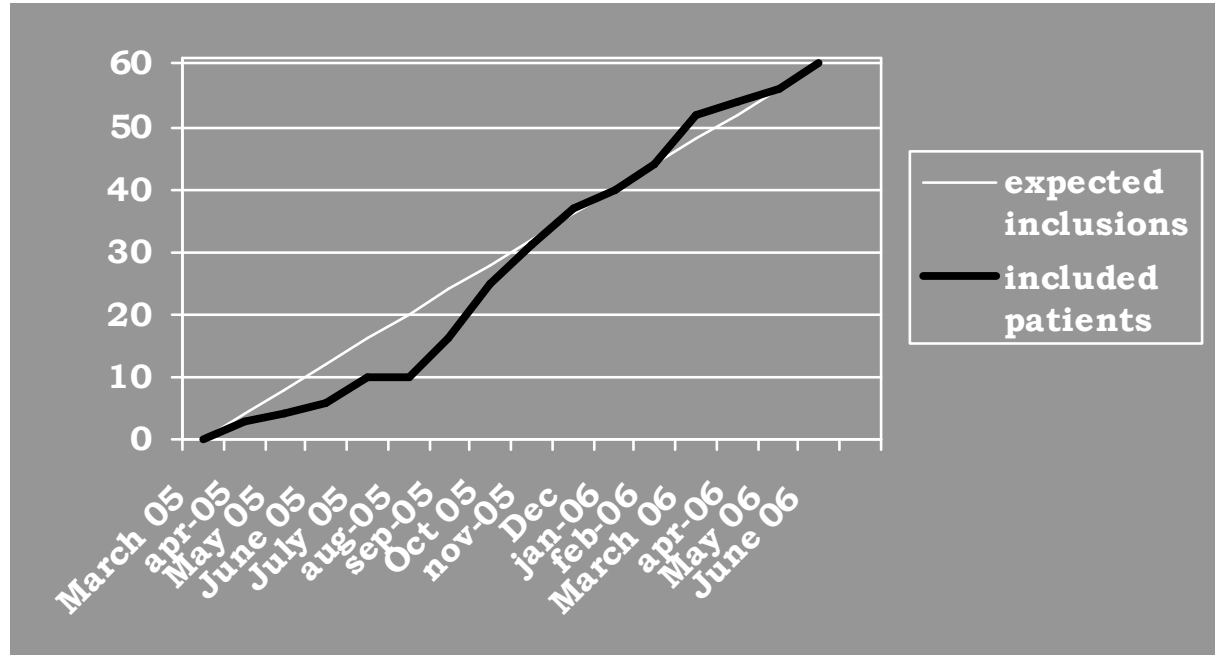

The second indicator of proper trial execution is that of the drop-out and loss-to-follow-up rates. Both were extremely low in all three trials. All three trials reported a $0 \%$ drop out and a loss-to-follow-up of less than $5 \%$. The trial on prolonged use of PT had been planned to include 
70 patients. In fact, inclusion was slightly less, mainly caused by a smaller than expected numbers of patients reaching less than $80^{\circ}$ of RoM in this period. The $0 \%$ loss-to-follow-up helped to achieve the calculated power, making additional inclusion unnecessary. As a result, we managed to complete the trial within the planned timeframe.

A third indicator of trial execution is compliance. In all three studies, compliance with the treatment protocol was good. In the clinical study on CPM, the mean time spent in the CPM machine was 3.6 hours, which was $90 \%$ of the target duration. In the study on prolonged CPM use, compliance was even better, with only one patient deviating from the suggested duration in the CPM machine. This patient spent less time in the CPM machine because her active RoM was over $100^{\circ}$ ! In the study on the efficiency of clinical PT, only two patients received one treatment less than scheduled.

One aspect that might be questionable is the follow-up duration used in the clinical CPM study. A between-group difference was found in the last follow-up assessment 17 days after surgery. No further follow-up assessment was included to check whether this difference would remain and thus lead to permanent differences in the further course of recovery, or whether differences would disappear over time. The results of the post-clinical CPM study, however, suggest that short-term benefits will diminish over longer term follow-up.

\section{Intention-to-treat analysis}

Statistical analyses were based on the intention-to-treat principle: all patients remained in the group to which they were assigned at randomization. Patients did not change groups during the treatment or followup phase in any of the studies. No scores were substituted for the patients who missed an outcome assessment, since we believe that the absence of data in the few missing cases was independent of the outcome or the treatment involved ${ }^{2122}$.

\section{Similarities and differences between the three RCTs}

All three studies included patients with osteoarthritis undergoing primary total knee arthroplasty. Inclusion and exclusion criteria for all studies were similar. The only extra inclusion criterion in the RCT on prolonged CPM use was a restriction on RoM and the fact that the patients had to reside in the Maastricht Heuvelland region. Although newly developed CPM machines are computerized and the settings on the machines can be changed according to views on the best rehabilitation protocol, we chose to keep our CPM treatment protocol simple in both CPM studies, since it is unclear what major factors influence effectiveness. Patients exercised for four hours daily. RoM was set according to tolerance and the machine moved from extension to flexion at a comfortable speed selected by the patient. No pauses were used in maximum flexion or extension position. 
Our RCT on the in-hospital use of CPM used the Hospital of Special Surgery Score as the functional outcome measure. In the later trials on prolonged CPM use, the outcome assessment included the Knee Society Score and the Western Ontario McMaster function, pain and stiffness scores (WOMAC). The primary reason for the change of outcome tool was that the HSS was considered to be inferior ${ }^{23} 24$ to the other measures. Although the WOMAC is reported to be more responsive ${ }^{25}$ than the KSS in the long term, both were included because no data exists on the short-term responsiveness of these two scales. Since physiotherapy is part of the rehabilitation of patients in the first few months after TKA, we decided to use both scales. Another reason was that the WOMAC is not applicable at the time of hospital discharge, because it is impossible to score several items of the scale at this time.

In the first trials, flexion and extension RoM were analyzed separately. In the final study, patients were included and analyzed on the basis of total RoM (flexion to extension). The decision to use full RoM as the outcome in this study resulted from new views on the effects of CPM, based on reports of negative effects of CPM on extension RoM $1526-28$.

Since a fixed time-span for inclusion was used in the RCT on inhospital CPM use, the number of patients required to achieve statistical significance was not determined for this study. We aimed for a large difference in treatment effect between the experimental and control interventions in the efficiency study. We discussed the potential benefits of doubling the treatment intensity, and agreed upon a between-group difference of $10^{\circ}$ being associated with a large treatment effect.

Based on the results of our earlier studies and the idea that differences smaller than $5^{\circ}$ are of no clinical importance, a smaller between-group difference was aimed at in the study on prolonged use of CPM.

Our RCTs on in-hospital CPM treatment and the efficiency of PT yielded similar results in terms of RoM measured at hospital discharge $\left(77^{\circ}\right.$ vs $78^{\circ}$ ). Mean RoM at discharge in the prolonged use of CPM trial was lower $\left(71^{\circ}\right)$, since this study included only patients with less than $80^{\circ}$ of RoM.

Results at 17 days, however, were only slightly less than those of the outcome assessment of the earlier trial on in-hospital CPM use (mean RoM $86^{\circ}$ vs $84^{\circ}$ ). Patients in the intervention group progressed more than patients in the control group and their RoM values caught up with those of the patients in the earlier trials, where no strict RoM criterion was used for inclusion.

At follow-up, however, the outcomes of the groups in terms of mean RoM differed from our previous study data (mean RoM $98^{\circ}$ vs $90^{\circ}$ in the prolonged CPM study). This would suggest that the use of CPM speeds up initial RoM improvement. After termination of the treatment, further improvement in RoM seems to follow the 'old' pattern again, e.g. in that patients with limited flexion at discharge progressed more slowly. 
Chapter 6 describes the design of our study on the effectiveness of prolonged use of CPM, as an adjunct to PT, following TKA. Publishing the design of a study before the results are available has various advantages. Information about a design is valuable because it allows a critical assessment of the methods and choices made in the design phase of a trial, independent of study outcome. Secondly, it may counteract possible publication bias, i.e., a study producing positive results seems more likely to be published than a study finding no difference between study groups 29 30. Thus, publication of the trial design makes other researchers in the field aware of studies in progress or being conducted on certain topics, even if they are not published. Thirdly, presentation of the design before results become available stops researchers from adjusting the design according to the outcome of the study. When results are contrary to the researcher's expectations, methodological flaws are examined thoroughly. However, if results are in line with expectations, methodological flaws are more likely to be overlooked.

\section{Implications for clinical practice}

We concluded from our systematic review that there is a moderate, positive, short-term effect of adding CPM to standardized PT after total knee arthroplasty, but that there is no relevant long-term benefit of CPM use. The results indicate that the effect of CPM treatment is large when CPM is compared to immobilization. The more aggressive the control treatments in terms of RoM exercises, however, the smaller the differences found. Compared to other regimens focusing on RoM, studies report no additional effect of CPM treatment.

Short-term effects of CPM treatment were found in both of our CPM trials. Our in-hospital PT programme focuses on functional recovery. Although RoM exercises are part of every treatment session, these exercises are not the only RoM enhancing component, since all patients also receive 4 hours of CPM treatment. Critics might call our basic programme conservative in terms of RoM exercises, and this conservative approach could explain the small effect of CPM we found over the basic treatment procedure.

It might thus be concluded that improvement of joint mobility could be speeded up by incorporating an intensive RoM treatment modality in a rehabilitation protocol. This modality might consist of CPM, although other modalities such as a slider board or a 'drop and dangle' regimen might be just as effective.

As regards the intensity of the in-hospital PT treatment, one may question what the main treatment goals for in-hospital PT should be. Since our trial on treatment intensity reported no dose-response relationship with RoM or functional outcome, it may be questioned whether improvement of RoM and function are eminent in-hospital treatment goals.

Either one PT session per day is really the optimal dose, or no major added effects on RoM are achieved by extra PT sessions when added to 4 hours of CPM use in the first few days after surgery. 
It may well be that the main treatment goals for the in-hospital situation can be found in terms of the time needed to reach functional goals like walking, stair climbing and independence in transfer from sitting to standing. Our efficiency study showed that these goals are generally reached within four days with one daily treatment session. As for the duration of CPM use, I believe that CPM should generally be used in the in-hospital setting. Only in cases with higher risks of RoM limitations (marked functional limitations, excessive pain, limited RoM prior to surgery) ${ }^{31-36}$ might CPM play a role in later stages of rehabilitation.

Results obtained in the studies reported in this thesis are generalizable to populations, cultures and health care systems comparable to the Dutch system. An example of a population where this generalizability may be problematic is that of patients living in the Middle East. In the past 6 years, I have supervised the short-term recovery of over 100 patients in this region (United Arab Emirates). If, for example, we had to apply the same inclusion criteria of our study on prolonged use of CPM to this population, we would have to exclude more than $90 \%$ of these patients. In all cases, the reason for exclusion would be that these patients all exceeded $80^{\circ}$ of RoM on the fourth day after surgery. Patients in the Middle East generally tend to perform better in terms of early postoperative RoM, whereas their progression of functional recovery is slower, which may partly be caused by cultural and social differences. Prior to surgery, RoM is larger than in our Maastricht population, the prime reason being that patients try to maintain deep knee flexion as long as possible for religious and social reasons. Contrary to the rapid improvement in RoM, however, the Middle East patients are less active than our population, leading to slower improvement in functional independence.

Regaining independence as fast as possible is regarded as being of eminent importance in our society and protocols. Social support and a more tranquil lifestyle seem to make fast functional independence less important in the Middle East.

\section{Suggestions for future research}

Many interesting aspects of the peri-operative care of TKA patients need further attention. In view of the findings of our systematic review, future research should adhere to the recommendations of the CONSORT statement when reporting results of randomized controlled trials. Special attention should be given to a detailed description of the interventions.

With regard to CPM treatment, future research might focus on assessing the differences in CPM effectiveness resulting from different application characteristics, such as intensity of CPM interventions and machine characteristics. In addition, the effect of CPM when combined and compared with various PT regimes may be studied further, since PT is more than CPM alone and not all PT programmes are alike.

Regarding other RoM enhancing methods, the effect of patellar mobilization on RoM may be another topic for future research, as may 
be the need for passive and aggressive traction and translation techniques in mobilizing the tibiofemoral joint.

Researchers could also investigate whether patients with certain profiles benefit more from CPM than others. Patients with limited RoM, limited functional capacity or relevant co-morbidity before surgery might benefit more from CPM treatment. The same holds for the overall treatment efficacy. Research into patient profiles might show how to identify those in need of more extensive or intensive rehabilitation schemes. Surveying the domain of physical therapy, there are other factors that influence outcome after TKA. Next to the patients' profile, social support may also play a role in postoperative recovery. Knowledge about the influence of these factors is lacking and this might also be a useful topic of future research.

To facilitate the interpretation and comparison of the results of various studies in TKA patients, additional research is needed to find better outcome tools. Studies might focus on outcome measures, and investigate whether RoM and limitations in functional capacity, assessed by means of questionnaires, really are the best measures available. A possible, more novel, outcome tool might be scales measuring participation. If these become available, it might be interesting to see whether certain treatments are superior in terms of their effects on participation.

Future research might also focus on overall postoperative PT care. Intensive exercise programmes are a part of many perioperative regimes. However, there is little evidence that these programmes are effective, simply because studies on integrated PT programmes are lacking. Future studies should investigate not only the content of these programmes but also the context in which they could be most effectively administered. A question to be answered may be whether, in time, patients can practice alone after being instructed by a physical therapist or whether they need extensive guided programmes. It might be interesting to assess whether self-management programmes are more effective in the long term because these programmes adress patients' self-effectiveness, which may be of great importance to their overall long-term recovery.

Last but not least, new developments in the field of orthopaedic surgery will have a major influence to impact on recovery after TKA. Aspects such as prosthesis design, operation techniques (computer navigation, minimally invasive surgery etc.) and surgeons' skills could all be topics for future research in TKA patients.

Although TKA is nowadays one of the most successful orthopaedic operations $^{1-3}$ it is obvious that there are still many topics that have not been fully examined, and much room for further improvement to benefit future patients. 


\section{References}

1. Brady OH, Masri BA, Garbuz DS, Duncan CP. Rheumatology: 10. Joint replacement of the hip and knee--when to refer and what to expect. Cmaj 2000;163(10):1285-91.

2. Ethgen O, Bruyere O, Richy F, Dardennes C, Reginster JY. Health-related quality of life in total hip and total knee arthroplasty. A qualitative and systematic review of the literature. J Bone Joint Surg Am 2004;86A(5):963-74.

3. Insall JN, Scott W, Scuderi GR. Current concepts in primary and revision total knee arthroplasty. Philidelphia: Lippincot-Raven, 1996.

4. Oxman AD, Guyatt GH. The science of reviewing research. Ann N Y Acad Sci 1993;703:125-33; discussion 133-4.

5. Antman EM, Lau J, Kupelnick B, Mosteller F, Chalmers TC. A comparison of results of meta-analyses of randomized control trials and recommendations of clinical experts. Treatments for myocardial infarction. Jama 1992;268(2):240-8.

6. van Tulder MW, Assendelft WJJ. Method guideline for systematic reviews in the Cochrane collaboration back review group for spinal disorders. Spine 1997;22(20):2323-2330.

7. Begg C, Cho M, Eastwood S, Horton R, Moher D, Olkin I, et al. Improving the quality of reporting of randomized controlled trials. The CONSORT statement. Jama 1996;276(8):637-9.

8. Altman DG. Better reporting of randomised controlled trials: the CONSORT statement. Bmj 1996;313(7057):570-1.

9. Colwell CW, Jr., Morris BA. The influence of continuous passive motion on the results of total knee arthroplasty. Clin Orthop Relat Res 1992(276):225-8.

10. Nielsen PT, Rechnagel K, Nielsen SE. No effect of continuous passive motion after arthroplasty of the knee. Acta Orthop Scand 1988;59(5):5801 .

11. Chen B, Zimmerman JR, Soulen L, DeLisa JA. Continuous passive motion after total knee arthroplasty: a prospective study. Am J Phys Med Rehabil 2000;79(5):421-6.

12. Chiarello CM, Gundersen L, O'Halloran T. The effect of continuous passive motion duration and increment on range of motion in total knee arthroplasty patients. J Orthop Sports Phys Ther 1997;25(2):119-27.

13. Harms M, Engstrom B. Continuous Passive Motion as an Adjunct to Treatment in the Physical Therapy Management of the Total Knee Arthroplasty Patient. Physiotherapy. 1991;77(April ):301-7.

14. McInnes J, Larson MG, Daltroy LH, Brown T, Fossel AH, Eaton HM, et al. A controlled evaluation of continuous passive motion in patients undergoing total knee arthroplasty. Jama 1992;268(11):1423-8.

15. Pope RO, Corcoran S, McCaul K, Howie DW. Continuous passive motion after primary total knee arthroplasty. Does it offer any benefits? $J$ Bone Joint Surg Br 1997;79(6):914-7.

16. Vince KG, Kelly MA, Beck J, Insall JN. Continuous passive motion after total knee arthroplasty. J Arthroplasty 1987;2(4):281-4.

17. Walker RH, Morris BA, Angulo DL, Schneider J, Colwell CW, Jr. Postoperative use of continuous passive motion, transcutaneous electrical nerve stimulation, and continuous cooling pad following total knee arthroplasty. J Arthroplasty 1991;6(2):151-6. 
18. Moher D, Cook DJ, Jadad AR, Tugwell P, Moher M, Jones A, et al. Assessing the quality of reports of randomised trials: implications for the conduct of meta-analyses. Health Technol Assess 1999;3(12):i-iv, 1-98.

19. Bouter LM, van Dongen MCJM, Zielhuis GA. Epidemiologisch onderzoek : opzet en interpretatie 5ed. Houten Bohn Stafleu Van Loghum, 2005.

20. Medical, Research, Counsil. Cluster randomised trials - Methodological and ethical considerations. London: Medical Research Council, 2002.

21. Little RJ. Methods for handling missing values in clinical trials. $J$ Rheumatol 1999;26(8):1654-6.

22. Hollis S, Campbell F. What is meant by intention to treat analysis? Survey of published randomised controlled trials. Bmj 1999;319(7211):670-4.

23. Davies DM, Johnston DW, Beaupre LA, Lier DA. Effect of adjunctive range-of-motion therapy after primary total knee arthroplasty on the use of health services after hospital discharge. Can J Surg 2003;46(1):30-6.

24. Insall JN, Dorr LD, Scott RD, Scott WN. Rationale of the Knee Society clinical rating system. Clin Orthop Relat Res 1989(248):13-4.

25. Lingard EA, Katz JN, Wright RJ, Wright EA, Sledge CB. Validity and responsiveness of the Knee Society Clinical Rating System in comparison with the SF-36 and WOMAC. J Bone Joint Surg Am 2001;83-A(12):185664.

26. Ritter MA, Gandolf VS, Holston KS. Continuous passive motion versus physical therapy in total knee arthroplasty. Clin Orthop Relat Res 1989(244):239-43.

27. Ververeli PA, Sutton DC, Hearn SL, Booth RE, Jr., Hozack WJ, Rothman RR. Continuous passive motion after total knee arthroplasty. Analysis of cost and benefits. Clin Orthop Relat Res 1995(321):208-15.

28. Laskin RS, Beksac B. Stiffness after total knee arthroplasty. $J$ Arthroplasty 2004;19(4 Suppl 1):41-6.

29. Ostelo RW, Koke AJ, Beurskens AJ, de Vet HC, Kerckhoffs MR, Vlaeyen JW, et al. Behavioral-graded activity compared with usual care after firsttime disk surgery: considerations of the design of a randomized clinical trial. J Manipulative Physiol Ther 2000;23(5):312-9.

30. Dickersin K. The existence of publication bias and risk factors for its occurrence. Jama 1990;263(10):1385-9.

31. Annouchi YS, McShane M, Kelly jr F, Elting J. Range of motion in total knee replacement. Clin Orthop Relat Res 1996;331:87-92.

32. Ritter MA, Berend ME, Harty LD, Davis KE, Meding JB, Keating EM. Predicting range of motion after revision total knee arthroplasty: clustering and log-linear regression analyses. $J$ Arthroplasty 2004;19(3):338-43.

33. Jones CA, Voaklander DC, Suarez-Alma ME. Determinants of function after total knee arthroplasty. Phys Ther 2003;83(8):696-706.

34. Lingard EA, Katz JN, Wright EA, Sledge CB. Predicting the outcome of total knee arthroplasty. J Bone Joint Surg Am 2004;86-A(10):2179-86.

35. Kim S, Losina E, Solomon DH, Wright J, Katz JN. Effectiveness of clinical pathways for total knee and total hip arthroplasty: literature review. $J$ Arthroplasty 2003;18(1):69-74.

36. Kotani A, Yonekura A, Bourne RB. Factors influencing range of motion after contemporary total knee arthroplasty. $J$ Arthroplasty 2005;20(7):850-6. 


\section{Summary}

Knee osteoarthritis (OA) is a common musculoskeletal condition affecting older people, causing pain, physical disability and reduced quality of life.

Total knee arthroplasty (TKA) is considered the treatment of choice for patients with intractable pain and substantial functional disabilities who have not had acceptable relief and functional improvement after conservative treatment.

Although there is general agreement that exercises completed after TKA promote walking and independence in activities of daily living, the relative advantages of peri-operative rehabilitation programmes in this population have not been extensively documented.

This thesis presents the results of several studies on different aspects of physiotherapy (PT) treatment in the perioperative care of TKA patients. The key issue in this thesis is the role of continuous passive motion (CPM) in perioperative care.

Chapter 1 provides an overview of the role of PT in the different perioperative phases and indicates the positions of the various chapters of the thesis in this overview.

Chapter 2 reports on a systematic review summarising the effect on range of motion of continuous passive motion (CPM) treatment in TKA patients. Relevant papers were identified by means of computer aided searches of Medline (1966 to July 2002), the Cochrane Controlled Trials Register, Cinahl and Embase (1988 to July 2002). In addition, references given in relevant publications were checked. Two reviewers independently assessed the methodological quality of the selected RCTs, using a standardised checklist for internal validity (the MaastrichtAmsterdam consensus list). Findings were summarised by means of a meta-analysis or by a rating system indicating four levels of evidence.

The review included fifteen studies investigating the effect of CPM in the management of TKA patients. Study quality ranged from poor $(2$ points on a 10-point scale) to good (8 points). The trials included in the review used different contrasts. Nine studies compared standardised PT plus CPM with a standardised rehabilitation programme. Two studies compared CPM with a physiotherapy programme. One study compared CPM with cast immobilisation. Two studies compared CPM with the use of a slider board. Finally, one study compared CPM plus Standard PT with PT plus a "drop and dangle" regimen.

The evidence found on the efficacy of CPM after TKA was conflicting. CPM treatment was superior to immobilisation, whereas CPM was equally successful as other active joint mobilisation programmes, like a drop and dangle regime and slider board treatment.

We found positive short-term effects of adding CPM to standardised PT. Five studies were included in a pooled analysis, for a total of 317 patients. The pooled effect size was $8.27^{\circ}$ (WMD), 95\% CI $-1.60,18.15$ ). No long-term effects of CPM use were found. 
We concluded that there is evidence of a moderate positive, short-term effect of addingCPM to standardised PT after total knee arthroplasty, but that there is no relevant long-term effect of CPM use.

Chapter 3 presents the design and results of a randomized controlled trial on the efficacy of CPM when added to PT in the in-hospital phase after total knee reconstruction. The objective of this study was to explore whether reported effects of CPM in older studies still held in a new postoperative rehabilitation regime, in which the in-hospital period was limited to five days.

Two exercise programmes were carried out during a 5-day postoperative period on an inpatient basis, starting on the first day after surgery. Outcome was assessed at the end of the hospital stay and two weeks after discharge. The main outcome measures were mobility and function, while secondary outcome measures included muscle strength, pain, satisfaction and length of hospital stay. From January to May 2001, 40 patients were included in this study. They were randomly allocated to either a group receiving 4 hours of CPM per day in addition to PT or a group receiving PT alone.

At the end of the treatment period we found a clear trend in favour of the CPM group in terms of range of motion (RoM), function measured with the Hospital of Special Surgery score, pain and muscle strength. The differences in RoM, function and pain persisted at follow-up. The overall RoM difference on day 17 was more than $10^{\circ}$. The results indicate that CPM still contributes to the improvement of RoM and function in modern rehabilitation regimes. In addition to an improved RoM, a protocol including CPM seems to have favourable effects on pain and muscle strength in the first two weeks after surgery.

Chapter 4 evaluates the interobserver reproducibility of the assessment of active and passive RoM, as measured by two experienced physiotherapists in 30 patients during the in-hospital phase after TKA. Both therapists, independently and in random order, assessed knee RoM on the third or fourth day after surgery. A long-arm goniometer was used to measure flexion and extension RoM. To quantify reproducibility, we distinguished between measures of agreement and measures of reliability. Measures of agreement refer to the absolute measurement error (presented in the units of measurement of the instrument) that is associated with one measurement taken from an individual patient. Measures of agreement thus provide insight into the ability of two or more observers to achieve the same value. Measures of reliability refer to the relative measurement error, i.e. the variation between patients in relation to the total variance of the measurements. They provide insight into the ability of two or more observers to differentiate between subjects in a group.

The reliability of RoM measurement was fair to good for all movements (Intraclass Correlation Coefficients ranging from 0.62 for the measurement of passive extension to 0.89 for the measurement of active flexion). Interobserver agreement for flexion as well as extension was only fair. 
The highest levels of agreement were found for measurement of passive flexion with the patient in sitting position and for measurement of passive extension (mean difference $2.7^{\circ}$; limits of agreement $-6.7^{\circ}$ to $12.1^{\circ}$ and mean difference $2.2^{\circ}$; limits of agreement $-6.2^{\circ}$ to $10.6^{\circ}$, respectively). Based on these results, the smallest detectable differences would be approximately $8^{\circ}$.

We concluded that when two different observers assess the same patients in the acute phase after total knee arthroplasty in a comparable clinical environment, differences in RoM of less than $8^{\circ}$ cannot be distinguished from measurement error. Reliability was found to be fair for extension RoM and good for flexion RoM measurements of the knee.

Chapter 5 discusses the results of a randomized controlled trial on the efficiency of immediate postoperative inpatient PT after total knee arthroplasty. Although aggressive rehabilitation is believed to be important, evidence of the effects of exercise programmes with different intensities after TKA is limited. Therefore, the main objective of this trial was to explore whether intensity of PT affects short term recovery, measured as RoM and function.

During the in-hospital phase, daily PT was administered to all participating patients. Patients in the experimental group were treated twice daily (totalling 40 minutes per day), while patients in the control group were treated once daily ( 20 minutes per day). A between-group difference of $10^{\circ}$ of passive RoM was considered clinically important enough to eventually raise the regular treatment frequency. Out of 55 subjects who were scheduled for surgery between January and June 2004, 43 were included in the study.

At the time of hospital discharge, no detectable difference between the experimental and control groups was found in terms of the primary outcome measure of RoM, nor in terms of any of the secondary outcome measures.

This study thus shows that in our university hospital setting, twice daily PT sessions do not produce detectably different results in terms of functions and activities, compared to daily PT sessions. It may be questioned whether multiple daily therapy sessions are needed as an inhospital PT regime in TKA patients.

Chapters 6 and 7 present the design and outcome of a trial on the effectiveness of prolonged use of CPM, when added to physiotherapy, following total knee arthroplasty. Although results from chapters 2 and 3 suggest that CPM should be implemented in the rehabilitation process after TKA surgery, there is substantial debate about the optimal period of CPM application. The objective of the research reported on in these chapters was to explore whether there is additional longer-term benefit of continuing CPM after hospital discharge.

In total, 60 patients were randomized. The experimental treatment consisted of 14 days of CPM use starting on the fifth day after surgery, added to usual PT care. The control treatment was usual PT care. The in-hospital rehabilitation scheme was identical for both groups. Primary outcome measures were improvement in RoM and functional recovery. 
Although we found a difference between the groups at the end of the active treatment period, we were unable to detect accompanying benefits in functional recovery. At follow-up, the short-term benefits in RoM were found to fade: neither at the six-week nor at the three-month follow-up could we detect any differences between the groups.

Our conclusion was therefore that although results indicate that prolonged CPM use has a short-term effect on RoM, routine implementation of prolonged CPM use in patients with limited RoM at hospital discharge should be reconsidered, since neither long-term effects nor transfer to better functional performance was detected

Finally, chapter 8 provides a general discussion on various aspects of the research reported on in this thesis. The chapter is divided into three sections. The first section discusses several aspects of the validity of the systematic review and our clinical trials. The second section presents non-methodological comments on the three RCTs. The chapter then concludes with a brief personal view on peri-operative PT. 


\section{Samenvatting}

Artrose is een aandoening van de gewrichten en wordt in het dagelijkse leven ook wel slijtage genoemd. Artrose kan in alle gewrichten optreden maar komt voornamelijk voor in de gewrichten van de handen, knieën en heupen. Onder ouderen is het de meest voorkomende gewrichtsaandoening. In totaal zijn er in Nederland meer dan 650.000 mensen die in meer of mindere mate last hebben van artrose. Artrose veroorzaakt pijn en fysieke beperkingen waardoor de door de patiënt ervaren kwaliteit van leven afneemt.

Indien de pijn en beperkingen in functioneren ernstig zijn en een conservatieve benadering geen verlichting van klachten meer geeft is een totale knie reconstructie, ook wel totale knie arthroplastiek (TKA) genoemd, de best overgebleven behandeloptie.

Hoewel algemeen wordt aangenomen dat fysiotherapie een rol speelt in de peri-operatieve begeleiding rondom implantatie van een TKA is er weinig wetenschappelijk bewijs voor effectiviteit van deze fysiotherapeutische begeleiding.

Dit proefschrift presenteert de resultaten van enkele studies waarin verschillende aspecten van de fysiotherapeutische begeleiding rondom de TKA zijn onderzocht. Drie studies naar effecten van behandeling via continue passief bewegen (continuous passive motion, CPM) in de perioperatieve begeleiding van TKA patiënten vormen het fundament van dit proefschrift.

Hoofdstuk 1 geeft allereerst een overzicht over de algemene rol van fysiotherapie in de verschillende peri-operatieve fasen rondom de totale knie arthroplastiek. Daarnaast wordt de plaats van verschillende hoofdstukken van dit proefschrift in deze peri-operatieve begeleiding verduidelijkt.

Hoofdstuk 2 beschrijft een systematisch literatuuroverzicht waarin studies worden samengevat die de effectiviteit, op bewegingsuitslag, hebben onderzocht van het gebruik van CPM in de nabehandeling van patiënten na een TKA. Om de relevante literatuur op te sporen zijn de volgende bibliografische databestanden doorzocht: Medline (1966-2002), Cochrane controlled trials register, Cinahl en Embase (1988-2002). Daarnaast zijn ook de referentielijsten van de relevante onderzoeken nagelopen. Uiteindelijk voldeden 15 gerandomiseerde gecontroleerde studies aan de inclusiecriteria. Twee onafhankelijke onderzoekers hebben de publicaties van deze studies op methodologische kwaliteit of wel interne validiteit beoordeeld aan de hand van een lijst van 10 criteria (Maastricht Amsterdam consensus lijst). Resultaten zijn samengevat met behulp van een meta-analyse. Indien uitvoeren van een meta-analyse niet mogelijk of niet zinvol was is het niveau van bewijskracht vastgesteld aan de hand van een systeem van beslisregels, bestaand uit 4 niveaus van bewijskracht. De methodologische kwaliteit varieerde van 2 tot 8 punten op een 10 puntenschaal. 
De RCT's vertoonden inhoudelijk nog veel verschillen. Allereerst met betrekking tot het contrast tussen de onderzochte interventies. In negen studies werd het verschil vergeleken tussen fysiotherapie en een behandeling waarbij CPM werd toegevoegd aan dezelfde behandeling. Twee studies vergeleken CPM met fysiotherapie. Een studie vergeleek CPM met immobilisatie. Twee studies vergeleken CPM met een ander programma dat de beweeglijkheid moet verbeteren, een zogenaamde 'slider board' . Ten slotte vergeleek een studie CPM behandeling met een zogenaamde 'drop and dangle' behandeling.

Het gevonden bewijs mag conflicterend genoemd worden. CPM behandeling is effectiever dan immobilisatie, maar vergelijkbaar met andere programma's waarin, naast standaard fysiotherapie, specifiek en uitgebreid aandacht geschonken wordt aan beweeglijkheid van de knie. Het bleek dat we alleen voor de toevoeging van CPM aan fysiotherapie genoeg vergelijkbare studies gevonden hadden om een meta-analyse uit te voeren. In deze meta-analyse zijn vijf studies met in totaal 317 deelnemers opgenomen. Het gepoolde korte termijn effect in het voordeel van de behandeling waaraan CPM was toegevoegd bleek $8.27^{\circ}$, 95\% betrouwbaarheidinterval -1,6-18,15). Op langere termijn werden geen effecten gevonden van toevoeging van CPM aan fysiotherapie.

Samenvattend kan gesteld worden dat er bewijs is voor een korte termijn effect van toevoeging van CPM aan een standaard fysiotherapeutische behandeling in de direct postoperatieve fase na een TKA. Er mag echter geen lange termijn effect verwacht worden van deze toevoeging.

Hoofdstuk 3 beschrijft het design en de resultaten van een gerandomiseerd gecontroleerd onderzoek naar de effecten van toevoegen van CPM behandeling aan fysiotherapie in de klinische fase na een implantatie van een TKA. Doelstelling van dit onderzoek was om na te gaan of beschreven effecten vanuit de systematische review ook aanwezig zijn in een modern nabehandelingprotocol waarin de ziekenhuisopname ingekort is tot 5 dagen.

De fysiotherapeutische behandeling was gestandaardiseerd, waarbij de nadruk lag op training van functionele vaardigheden als opstaan uit bed en stoel, gaan en traplopen, daarnaast was er aandacht voor beweeglijkheid van het kniegewricht en kracht en belastingsoefeningen. De toegevoegde CPM behandeling bestond uit 4 uren bewegen over een zo groot mogelijk bewegingstraject per dag. Het bewegingstraject werd individueel ingesteld op geleide van pijntolerantie van de patiënten.

De primaire effectmeting werd 4 dagen na de operatie uitgevoerd. Dit moment viel gelijk met het geplande tijdstip van ontslag. Follow-up meting vond plaats twee weken na ontslag uit het ziekenhuis. Beweeglijkheid en functioneren zijn gekozen als belangrijkste uitkomstmaten.

40 patiënten zijn in deze studie opgenomen in de periode januari tot mei 2001. Zij zijn door randomisatie toegewezen aan een experimentele groep die 4 uur per dag CPM behandeling kreeg, toegevoegd aan fysiotherapie of een controlegroep waarin de patiënten alleen fysiotherapie behandelingen kregen. 
Bij de primaire uitkomstmeting namen we een duidelijke trend waar op alle uitkomstmaten in het voordeel van de CPM groep. Zowel op beweeglijkheid als op functioneren, gemeten met de Hospital of Special Surgery score, pijn en spierkracht. De verschillen $\mathrm{n}$ beweeglijkheid, functioneren en pijn persisteren tijdens de follow-up meting twee weken na ontslag. Het gevonden verschil in beweeglijkheid bij follow-up was groter dan $10^{\circ}$.

De resultaten van dit onderzoek geven aan dat toevoeging van CPM aan een klinisch fysiotherapeutische behandeling ook een meerwaarde heeft, op korte termijn gemeten, in een modern behandelprotocol met korte opnameduur in het ziekenhuis.

Hoofdstuk 4 beschrijft de opzet en resultaten van een onderzoek naar de reproduceerbaarheid van metingen van de bewegingsuitslag van de knie in de klinische fase na een TKA.

Het doel van het onderzoek was enerzijds zicht te krijgen op de reproduceerbaarheid van metingen van de kniemobiliteit en anderzijds om aan te geven dat reproduceerbaarheid onderscheiden kan worden in betrouwbaarheid en overeenstemming. Twee ervaren fysiotherapeuten hebben in de periode januari- maart 2004 de bewegingsuitslag gemeten bij 30 TKA patiënten. Bij alle patiënten zijn de actieve en passieve bewegingsuitslagen gemeten op de derde of de vierde dag na de TKA. Voor de metingen zijn lange arm goniometers gebruikt. Metingen werden gestandaardiseerd uitgevoerd en de uitvoering is voor de start van het onderzoek getraind op gezonde vrijwilligers. Reproduceerbaarheid is onderverdeeld in meten van betrouwbaarheid en meten van overeenstemming. Maten van overeenstemming verwijzen naar de absolute meetfout weergegeven in de oorspronkelijke meeteenheid (graden beweeglijkheid) geassocieerd met een meting bij een individuele patiënt. Overeenstemming geeft inzicht in het vermogen van twee waarnemers om tot dezelfde uitkomst te komen. Maten van betrouwbaarheid refereren naar de relatieve meetfout. De variatie tussen patiënten in relatie tot de totale variantie van de metingen. Deze maten geven inzicht in het vermogen, tussen twee waarnemers of metingen, om personen te onderscheiden.

De betrouwbaarheid van beweeglijkheidmetingen blijkt redelijk tot goed voor zowel meting van flexie als extensie van de knie. De gevonden Intraclass correlatie coëfficiënten variëren van 0.62 voor meting van passieve extensie tot 0.89 voor de meting van actieve flexie.

Uitkomsten van interbeoordelaarsovereenstemming waren minder positief. De kleinste grenzen van overeenstemming zijn gevonden voor de meting van passieve flexie, gemeten met de patiënt in zit en de passieve extensiemetingen (Het gemiddelde verschil tussen twee metingen bedroeg $2.7^{\circ}$; grenzen van overeenstemming $-6.7^{\circ}$ tot $12.1^{\circ}$ en $2.2^{\circ}$ : grenzen van overeenstemming $-6.2^{\circ}$ tot $10.6^{\circ}$ ).

Gebaseerd op bovenstaande uitkomsten zal het kleinst meetbare verschil ongeveer $8^{\circ}$ bedragen. 
Dat betekent dat alleen verschillen groter dan $8^{\circ}$ gedetecteerd kunnen worden als verbetering groter dan de meetfout in de situatie waarin twee beoordelaars patiënten meten in de klinische fase na implantatie van een TKA.

In Hoofdstuk 5 worden de gehanteerde methodologie en de resultaten van een gerandomiseerd gecontroleerde studie naar de efficiëntie van klinische fysiotherapie na een TKA beschreven.

Hoewel algemeen verondersteld wordt dat intensief nabehandelen na een TKA belangrijk is, is er op dit moment nog geen ondersteunend bewijs voor deze gedachte. Gedurende de ziekenhuisopname krijgen patienten dagelijks fysiotherapie. De frequentie verschilt tussen een of meerdere behandelingen per dag. In dit onderzoek staat de vraag centraal of er verschil is in effect tussen twee en een maal daags fysiotherapeutisch behandelen in de klinische fase na implantatie van een TKA. De patiënten zijn in het onderzoek een of twee maal daags gedurende 20 minuten behandeld. Bij de opzet van het onderzoek zijn we uitgegaan van de gedachte dat een verschil tussen beide interventies van $10^{\circ}$ passieve beweeglijkheid groot genoeg is om aanpassing van het behandelbeleid te rechtvaardigen.

Van de 55 patiënten die in de periode januari tot juni 2004 zijn geopereerd zijn 43 patiënten geïncludeerd in deze studie. Ten tijde van de primaire uitkomstmeting, 4 dagen na de operatie is er geen verschil in beweeglijkheid, of enig andere uitkomstmaat, gevonden tussen beide behandelstrategieën. Deze studie toont aan dat, in de setting van een academisch ziekenhuis, twee maal daags behandelen geen meerwaarde heeft boven een maal daags behandelen, gemeten in functies en activiteiten.

Hoofdstukken 6 en 7 beschrijven het design en de uitkomsten van een derde gerandomiseerd gecontroleerde studie uit deze thesis. Doelstelling van deze studie was na te gaan of langduriger gebruik van CPM effectief is op verbetering van beweeglijkheid en functioneren bij patiënten met een primaire totale knie arthroplastiek ten gevolge van artrose.

Om deze vraag te beantwoorden zijn in de periode maart 2005 tot juni 200660 patiënten geïncludeerd. Patiënten met een grotere totale beweeglijkheid van $80^{\circ}$ in de knie, ten tijde van ontslag uit het ziekenhuis, zijn uitgesloten van deelname in deze studie. Belangrijkste reden hiervoor was de gedachte dat CPM meer effect zou kunnen hebben in een populatie met beperkte beweeglijkheid in de eerste dagen na de operatie. Na een identieke begeleiding in de klinische fase zijn de deelnemende patiënten gerandomiseerd over twee groepen. Patiënten in de experimentele groep kregen reguliere fysiotherapeutische behandeling waaraan 14 dagen gebruik van een elektrische slee is toegevoegd. CPM machines werden bij de patiënten thuis afgeleverd en de patiënten werd geadviseerd de behandeling te continueren volgens hetzelfde schema als gehanteerd in de klinische fase, wat 4 uren CPM gebruik per dag betekende. Patiënten in de controlegroep kregen alleen de reguliere fysiothe- 
rapeutische nabehandeling. Als primaire uitkomstmaten is gekozen voorbeweeglijkheid en functioneren.

Van de 147 TKA patiënten die in deze onderzoeksperiode geopereerd zijn in het 'Artrose Kliniek Maastricht programma zijn uiteindelijk 60 patiënten in deze studie geïncludeerd.

Wij vonden een duidelijke trend in verbetering van RoM op korte termijn. Patiënten in de CPM groep verbeterden gemiddeld $5^{\circ}$ meer in de actieve behandelperiode van twee weken, in vergelijking met de patiënten in de controlegroep. Bij follow-up metingen na 6 weken was deze initiële voorsprong in beweeglijkheid echter geheel verdwenen. De verbeterde beweeglijkheid in de actieve behandelperiode leidde niet tot detecteerbare verschillen in functioneren, gemeten met de WOMAC en de Knee Society Score.

Standaard toevoegen van CPM aan de postklinische nabehandeling van patiënten na een TKA met een beperkte RoM ten tijde van ontslag lijkt dan ook niet wenselijk.

Tot slot beschrijft hoofdstuk 8 een algemene discussie over verschillende aspecten van de verschillende studies in deze thesis. Het hoofdstuk is verdeeld in drie delen. Het eerste gedeelte gaat in op interne validiteit van de systematische review en de drie RCT's in dit proefschrift. Daarna worden enkele niet methodologische kanttekeningen geplaatst bij de drie RCT's. Ten slotte geef ik kort weer wat mijn eigen beeld is bij het onderzoek naar peri-operatieve fysiotherapie bij patiënten na implantatie van een totale knie prothese 


$$
128
$$




\section{Dankwoord}

Bij het schrijven van een proefschrift hoort ook het bedanken van personen die op een of ander wijze hebben bijgedragen aan het ontstaan ervan. Je schrijft het immers niet alleen, zoals de kaft doet vermoeden. Het is meer het gevolg van samenwerking met velen, zoals de auteurslijsten bij de verschillende hoofdstukken aangeven. Sterker nog, ook die lijsten zijn maar een slap aftreksel van alle personen die een bijdrage hebben geleverd aan de totstandkoming van dit proefschrift.

Enkele van die personen wil ik hier bijzonder bedanken.

Allereerst de deelnemers, zonder deelnemende patiënten zouden de verschillende onderzoeken nooit zijn uitgevoerd. Daarom wil ik alle deelnemers aan de verschillende studies bedanken voor hun medewerking. De toewijding waarmee jullie deelnamen resulteerde niet alleen in weinig uitval in de resultatensecties, maar zorgde ook voor aanhoudende motivatie van het onderzoeksteam. De hartelijke ontvangst tijdens de huisbezoeken in de laatste twee jaren droegen daar nog verder aan bij.

Rob de Bie, we werken al heel lang samen. Eind jaren ' 80 gezamenlijk als onderzoeksassistent in het Fibromyalgie project van reumatologie. Van 1993 tot 1998 was ik onderzoeksassistent in jouw promotieonderzoek. In 2001 verving ik Mariëlle in het 'PPBS' project en vervolgens was jij een natuurlijke keuze als promotor. Bedankt voor de gezelligheid, gedeelde visies, samenwerking en begeleiding door de jaren heen, ik hoop dat we in de toekomst nog vaker samen mogen werken.

Piet van den Brandt en Ruud Geesink, mijn beide andere promotoren wil ik bedanken voor het in mij gestelde vertrouwen. Piet, ik heb je snelle, en toch kritische, reacties op mijn stukken zeer gewaardeerd.

De leden van de beoordelingscommissie, prof. dr. van der Linden, prof. dr. Dinant, prof. dr. Kuipers, prof. dr. Oostendorp en prof. dr. Verhaar wil ik bedanken voor het lezen en beoordelen van mijn proefschrift.

Hans Sein en Tilman Blachut wil ik bedanken voor het ter beschikking stellen van de CPM machines in het onderzoek naar tijdsduur van CPM gebruik.

Yvonne Crijns, je was mijn assistente in meerdere projecten. Ik kon altijd op je rekenen als het nodig was en kon er zonder enige bedenkingen vanuit gaan dat jij het werk consciëntieus en klantvriendelijk zou doen. Bovendien ben je altijd goed gehumeurd en leergierig. Zonder jou zouden de verschillende studies nooit zo 'vlekarm' zijn verlopen.

Één bedankje is eigenlijk veel te weinig voor je werk! 
Edje, king of Ace, Waltjé. Yvonne deed haar werk zo goed dat jij slechts zelden moest inspringen. Toch mag jij in dit dankwoord niet ontbreken. We werken al vanaf 1987 samen bij de afdeling orthopedie en hebben in die jaren samen heel wat beleefd. Sfeer en plezier zijn belangrijke voorwaarden voor goed functioneren en jij bent in mijn ogen in grote mate bepalend voor de goede sfeer bij de afdeling fysiotherapie. Ed, je bent veel meer dan een collega en ik hoop dan ook dat we nog lang samen mogen werken.

Albère Köke, 20 jaar al rijden we samen naar Maastricht, bediscussiëren in die anderhalf uur heen en weer van alles en nog wat. Samen in de onderzoekscommissie en later alle twee studeren. Onze gesprekken hebben een grote bijdrage gehad aan de ontwikkeling van mijn visie op fysiotherapie en wetenschap, daarnaast was het natuurlijk een gezellige tijd. Ik hoop dan ook dat we onze discussies over het wel en wee van de fysiotherapie nog lang kunnen voortzetten.

Ik ben er dan ook trots op dat jij mijn paranimf wilt zijn.

Appie Wijnen, mijn andere paranimf. Eerst mentor en daarna langdurig collega in de knierevalidatie, daarnaast altijd steun en toeverlaat. Ook jouw bijdrage aan mijn ontwikkeling is groot. Eerst leerde je me kritisch kijken en later was je een toonbeeld van toewijding en klantgerichtheid. Je biljartkunsten in onze vroege jaren waren onnavolgbaar, misschien vandaar dat de "BC Zoepe en Stoete" ter ziele is gegaan.

George Roox, een bedankje voor jou is zeker op zijn plaats. Soms tegen de stroom in, hield jij vast aan de onderzoekstaak binnen de afdeling. Een resultaat hiervan ligt nu voor je! Zonder je vasthoudendheid was het mogelijk nooit zo ver gekomen.

Collega's bij de afdeling fysiotherapie azM. Het valt niet mee om, naast een hoge werkdruk ook nog lastig gevallen te worden door een onderzoeker die zo nodig projecten van de grond wil trekken. Hopelijk komt er de komende jaren meer tijd om ook andere gebieden van ons klinisch werk onder de loep te nemen.

Bedankt in ieder geval voor de mogelijkheid om de "orthopedietrein" te kunnen volgen en zo dit boekje samen te stellen.

Artsen en medewerkers van de afdeling orthopedie, bedankt voor het 'lenen' van patiënten voor mijn onderzoeken, voor jullie steun bij het werven van patiënten en in het voorkómen van 'loss to follow up' in de verschillende studies.

Jos Slangen en Harrie van Montfoort, bedankt voor de PC hulp, zonder jullie was het invoeren en ordenen van de gegevens veel lastiger geweest. Ook hebben we samen gemerkt dat bestellen van ICT benodigdheden niet altijd vlekkeloos verloopt en een tijdrovende activiteit kan zijn. 
Conny de Zwart, mijn link naar de vakgroep epidemiologie, bedankt voor al je hand- en spandiensten.

Yvonne Jaegers, toen het werk op zijn einde liep was jij er om het geheel in de juiste format te plaatsen. Jij hebt gemerkt dat meewerken soms ook tot meer-werken kan leiden.

Pim en Juliette, jullie aanwezigheid, drukte en aandacht werkten relativerend en zorgden voor ontspanning, jullie welzijn is veel belangrijker dan alle regels in dit proefschrift.

Tot slot, Birgitte. Ik heb lang volgehouden dat promoveren voor mij gewoon een logisch gevolg was van het doen van meerdere onderzoeken op eenzelfde terrein. De laatste periode nam de druk toch toe en dat was soms merkbaar. Promoveren is wèl meer dan alleen een logisch gevolg van een manier van werken. Bedankt voor je steun, luisterend oor, opbeurende woorden, begrip, verzorging en voor al die andere zaken die ik nu niet aan het papier toevertrouw.

Zonder jou was het niet gelukt en was het resultaat voor mij veel minder waardevol. 
132 


\section{Curriculum Vitae}

Ton Lenssen was born on November 22, 1961 in s-Hertogenbosch. After obtaining his secondary school diploma in 1979 at the 'Albert Schweizer scholengemeenschap' in Geleen, he studied Physiotherapy at the hogeschool Heerlen. After obtaining his B.Sc. degree in 1986 Ton worked as a Physiotherapist in several health care institutes in the Netherlands and Germany. From 1987 he is working at the physiotherapy department of the Maastricht university hospital (azM).

In 1998 he started his study at the department of Health Sciences of Maastricht University. After his graduation (Cum Laude) in 2001 he is working as physiotherapist/research coordinator at the department of physiotherapy of the azM. Since 2000 he is also working as a lecturer at the hogeschool Zuyd in Heerlen, where he is involved in teaching evidence based medicine.

The $\mathrm{PhD}$ project described in this thesis is a collection of different studies conducted in the last 6 years, working as physiotherapist/researcher at the department of physiotherapy, azM. 
Ton Lenssen werd geboren op 22 november 1961, te s-Hertogenbosch. $\mathrm{Na}$ zijn middelbare schoolopleiding gevolgd te hebben aan de Albert Schweizer scholengemeenschap te Geleen studeerde hij fysiotherapie aan de hogeschool Heerlen. Na het behalen van zijn diploma fysiotherapie in 1986, werkte hij kortdurend in verschillende fysiotherapie praktijken in Limburg en het Duitse grensgebied. Vanaf 1987 werkt hij bij de afdeling fysiotherapie in het academisch ziekenhuis Maastricht. Tussen 1989 en 1997 participeerde Ton meermaals in verschillende wetenschappelijke onderzoeken. Tussen 1998 en 2001 studeerde hij bewegingswetenschappen aan de Universiteit Maastricht. Na behalen van zijn Master titel (cum laude) werkt hij als fysiotherapeut-onderzoeker bij de afdeling fysiotherapie in het azM. Daarnaast werkt hij sinds 2000 als docent bij de opleiding fysiotherapie van de hogeschool Zuyd in Heerlen waar hij zich vooral richt op doceren van evidence based handelen en begeleiding van afstudeerprojecten van studenten fysiotherapie en studenten die de opleidingen bewegingswetenschappen en fysiotherapie combineren.

Deze thesis is opgebouwd uit studies die Ton in de afgelopen 6 jaar in zijn functie als fysiotherapeut-onderzoeker binnen het azM, heeft uitgevoerd. 


\section{List of publications}

1. Lenssen AF, Wijnen LCAM, Wouters YMS. Fysiotherapie bij conservatief behandelde voorste kruisband letsels. Nederlands Tijdschrift voor Fysiotherapie 1993;3:118-126.

2. Wijnen LCAM, Lenssen AF, Kuys-Wouters YMS, de Bie RA, Borghouts JAJ, Bulstra SK. Mc Connell-therapie versus Coumans bandage bij patellofemorale pijnklachten. Een gerandomiseerde pilotstudie. Nederlands Tijdschrift voor Fysiotherapie 1996;special:12-17.

3. de Bie RA, de Vet HC, van den Wildenberg FA, Lenssen AF, Knipschild PG. The prognosis of ankle sprains. Int J Sports Med 1997;18(4):285-9.

4. de Bie RA, de Vet HCAW, van den Wildenberg FJAM, Lenssen AF, Bouter L, Knipschild PG. Efficacy of laser therapy in ankle sprains: design of a randomized clinical trial. Laser therapy 1997;9:165-172.

5. de Bie RA, de Vet HC, Lenssen AF, van den Wildenberg FA, Kootstra G, Knipschild PG. Low-level laser therapy in ankle sprains: a randomized clinical trial. Arch Phys Med Rehabil 1998;79(11):1415-20.

6. de Bie RA, Hendriks HJM, Lenssen AF, Van Moorsel SR, Opraus KWF, Remkes WFA, et al. KNGF richtlijn acuut enkelletsel. Nederlands Tijdschrift voor Fysiotherapie 1998;108(1): supplement.

7. de Bie RA, Verhagen AP, Lenssen AF, de Vet HCAW, van den Wildenberg FAJM, Kootstra G, et al. Efficacy of 904 nm laser therapy in the management of musculoskeletal disorders: a systematic review. Physical Therapy Reviews 1998;3:59-72.

8. Verhagen AP, de Bie RA, Lenssen AF, de Vet HC, Kessels AG, Boers M, et al. Impact of quality items on study outcome. Treatments in acute lateral ankle sprains. Int $\mathrm{J}$ Technol Assess Health Care 2000;16(4):1136-46.

9. Verhagen AP, de Bie RA, Lenssen AF, de Vet HCW. Effectiviteit van 904 $\mathrm{nm}$ lasertherapie bij aandoeningen van het bewegingsapparaat: een systematische review. Nederlands Tijdschrift voor Fysiotherapie 2000;110:84-91.

10. Verhagen AP, De Bie RA, Lenssen AF, de Vet HCW, Kessels AGH, Boers M, et al. Quality assessment of trials: a comparison of three criteria lists. Physical Therapy Reviews 2000;5:49-58.

11. Lenssen AF, Köke AJA, De Bie RA. Effectiviteit van continuous passive motion in de klinische nabehandeling van patienten na een totale knie prothese: een systematische review. Nederlands Tijdschrift voor Fysiotherapie 2001; dec 2001(6):152-158.

12. de Bie RA, Lenssen AF, Ostelo RGO. Evidence based practice; Wetenschappelijke onderbouwing van de fysiotherapiepraktijk Onderwijs in wetenschap Houten: Bohn Stafleu Van Loghum, 2002:3-7.

13. de Bie RA, Lenssen AF, Hendriks E. Het nut van een functiescore bij acuut enkelletsel. Stimulus 2003;2:202-205.

14. de Bie RA, Heemskerk MAMB, Stomp DJ, Lenssen AF, Van Moorsel SR, Rondhuis G, et al. KNGF richtlijn chronisch enkelletsel. Nederlands Tijdschrift voor Fysiotherapie 2003;2(Supplement).

15. Feyts YAEJ, Bongaarts L, Lenssen AF, Wolters PMJC. Effectiviteit van actieve oefentherapie bij chronisch hartfalen. Een systematische review. Nederlands Tijdschrift voor Fysiotherapie 2003;113:75-85.

16. Lenssen AF, Bie de RA, Bulstra SK, Steijn van MJA. Continuous passive motion in rehabilitation following total knee arthroplasty: a Randomised controlled trial. Physical Therapy Reviews 2003;8(3):123-129. 
17. Lenssen AF, Koke AJA, Bie de RA, Geesink RJT. Continuous passive motion in rehabilitation following total knee arthroplasty; short and long term effects on range of motion. Physical Therapy reviews 2003;8(3):113-121.

18. Lenssen AF, Wijnen, LCAM Fysiotherapeutische behandeling bij anterieure kniepijn Jaarboek fysiotherapie 2003. Houten: Bohn Stafleu Van Loghum, 2003:121-138.

19. Rijks CPM, Lenssen AF, de Bie RA . De richtlijn 'peri operatieve longfysiotherapie bij buik en hartchirurgie': een screening nader bekeken. Nederlands Tijdschrift voor Fysiotherapie 2003;113:75-81.

20. Cuypers CJT, Nelissen LH, Lenssen AF. Onderzoek naar de intra- en interbeoordelaarsbetrouwbaarheid van de Nederlandse versie van de Elderly mobility scale toegepast bij geriatrische patiënten in de klinische fase. Nederlands Tijdschrift voor Fysiotherapie 2004;114:110-113.

21. Lenssen AF. Efficiënt literatuur zoeken, gebruik van 'clinical queries' in Pubmed. Stimulus 2004;4:340-355.

22. Lenssen AF, Wijnen LCAM. Fysiotherapeutische behandeling bij anterieure kniepijn Stimulus 2005;4:440-456.

23. Smidt N, de Vet HC, Bouter LM, Dekker J, Arendzen JH, de Bie RA, et al. Effectiveness of exercise therapy: a best-evidence summary of systematic reviews. Aust J Physiother 2005;51(2):71-85.

24. Smidt N, de Vet HC, Bouter LM, Dekker J, Arendzen JH, de Bie RA, et al. De effectiviteit van oefentherapie: een review van systematische reviews. Nederlands Tijdschrift voor Fysiotherapie 2005;115(4):86-95.

25. van der Burgt G, Orsouw CM, Nelissen LH, Theunissen CTH, Lenssen AF. Klinimetrie bij CVA patienten: een onderzoek naar de intrabeoordelaarsbetrouwbaarheid van de trunk control test, de functional ambulation categories en de 10 meter looptest. . Nederlands Tijdschrift voor Fysiotherapie 2005;115(6):154-157.

26. Heymans MW, Lenssen AF, de Vet HCW. Lesbrief beschrijvende statistiek. Nederlands Tijdschrift voor Fysiotherapie 2006;116(3):68-71.

27. Lenssen AF. rubriek 'Meten in de praktijk': Knee Society clinical rating Scale. Nederlands Tijdschrift voor Fysiotherapie 2006;116(3):72.

28. Lenssen AF, Crijns YH, Waltje EM, Roox GM, van Steyn MJ, Geesink RJT, et al. Effectiveness of prolonged use of continuous passive motion (CPM) as an adjunct to physiotherapy following total knee arthroplasty: design of a randomised controlled trial [ISRCTN85759656]. BMC Musculoskelet Disord 2006;7:15.

29. Lenssen AF, Crijns YH, Waltje EM, van Steyn MJ, Geesink RJT, van den Brandt PA, et al. Efficiency of immediate postoperative inpatient physical therapy following total knee arthroplasty: an RCT. BMC Musculoskelet Disord 2006; 7:71.

30. Wees van der PJ, Lenssen AF, Hendriks EJ, Stomp DJ, Dekker J, de Bie RA. Effectiveness of exercise therapy and manual mobilisation in ankle sprain and functional instability: a systematic review. Aust J Physiother 2006;52(1):27-37.

31. Heymans MW, Lenssen AF, Vet HCW. Lesbrief toetsende statistiek. Nederlands Tijdschrift voor Fysiotherapie 2006;116:98-101.

32. Van der Wees PJ, Lenssen AF, Feijts YAEJ, Bloo H, Van Moorsel SR, Ouderland R, et al. KNGF richtlijn enkelletsel. Nederlands Tijdschrift voor Fysiotherapie 2006; jaargang 116:5(5): Supplement. 
33. Neeleman-van der Steen CWM, Rondhuis G, Moorsel SR, Brooijmans F, Lenssen AF, Hullegie W, et al. KNGF richtlijn Meniscectomie. Ned Tijdschr Fysiotherapie 2006;116:6(6): Supplement.

34. Lenssen AF, de Bie RA. Role of physiotherapy in peri-operative management in total knee and hip surgery. Injury 2006;37(S):S43-S45. 